Portland State University

PDXScholar

Spring 6-5-2017

\title{
Design and Application of a 3D Photocatalyst Material for Water Purification
}

Simon Paul Fowler

Portland State University

Follow this and additional works at: https://pdxscholar.library.pdx.edu/open_access_etds

Part of the Physics Commons, and the Water Resource Management Commons Let us know how access to this document benefits you.

\section{Recommended Citation}

Fowler, Simon Paul, "Design and Application of a 3D Photocatalyst Material for Water Purification" (2017). Dissertations and Theses. Paper 3648.

https://doi.org/10.15760/etd.5532

This Dissertation is brought to you for free and open access. It has been accepted for inclusion in Dissertations and Theses by an authorized administrator of PDXScholar. Please contact us if we can make this document more accessible: pdxscholar@pdx.edu. 
Design and Application of a 3D Photocatalyst Material for Water Purification

$$
\text { by }
$$

Simon Paul Fowler

\begin{abstract}
A dissertation submitted in partial fulfillment of the requirements for the degree of
\end{abstract}

\author{
Doctor of Philosophy \\ in \\ Applied Physics
}
Dissertation Committee:
Jun Jiao, Chair
Andrea Goforth
Andres La Rosa
Raj Solanki

\title{
Portland State University


(C) 2017 Simon Paul Fowler 


\begin{abstract}
This dissertation presents a method for enhancement of the efficiency and scalability of photocatalytic water purification systems, along with an experimental validation of the concept. A 3-dimensional photocatalyst structure, made from a $\mathrm{TiO}_{2}-\mathrm{SiO}_{2}$ composite, has been designed and fabricated for use in a custom designed LED-source illumination chamber of rotational symmetry that corresponds with the symmetry of the photocatalyst material. The design of the photocatalyst material has two defining characteristics: geometrical form and material composition. The design of the material was developed through the creation of a theoretical model for consideration of the system's photonic efficiency. Fabrication of the material was accomplished using a Ti alkoxide solution to coat a novel 3D support structure. The coatings were then heat treated to form a semiconducting thin-film. The resulting films were evaluated by SEM, TEM, UV-vis spectroscopy and Raman spectroscopy. The surface of the material was then modified by implantation of $\mathrm{TiO}_{2}$ and $\mathrm{SiO}_{2}$ nanoparticles in order to increase catalytic surface area and improve the photoactivity of the material, resulting in increased degradation performance by more than $500 \%$. Finally, the efficiency of the photocatalytic reactor was considered with respect to energy usage as defined by the Electrical Energy per Order (EEO) characterization model. The effects of catalyst surface modification and UV-illumination intensity on the EEO value were measured and analyzed. The result of the modifications was an $81.9 \%$ reduction in energy usage. The lowest EEO achieved was $54 \mathrm{kWh}$ per cubic meter of water for each order of magnitude reduction in pollutant concentration an improvement in EEO over previously reported thin-film based photoreactors.
\end{abstract}




\section{Dedication}

I would like to dedicate this work to my family. Especially, to my brilliant and loving partner, Anna Blum, who has been so incredibly supportive through the years that this work required, to my caring mother and father, Kathy and Steve, and my incredible siblings, Poppy and Andrew, whom I adore. I am lucky to have you all in my life and don't know who I would be without you. 


\section{Acknowledgements}

There were many helping hands in the course of this research. I would like to acknowledge all that contributed to this project: Dr. Jun Jiao, and the entire Jiao Research Group, especially Ryan Catabay, Anthea Leng, Esteban Rodriguez-Ariza, Emilio Molina, Sophia Weeks, Eleanor Solomon, Sydney Quinton-Cox, and Peter Lommen, who participated directly in the experimentation. Funding for this research was, of course crucial to its success, and I would like to thank Ken Gunther, the PSU Institute of Sustainable Solutions, and the PSU University Venture Development Fund for their contributions. 


\section{Table of Contents}

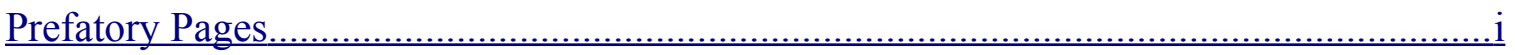

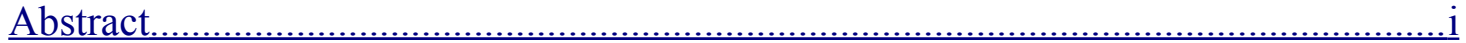

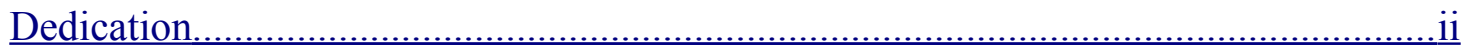

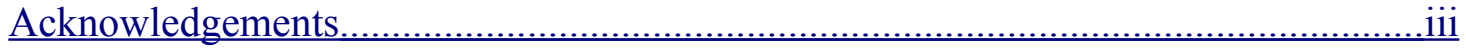

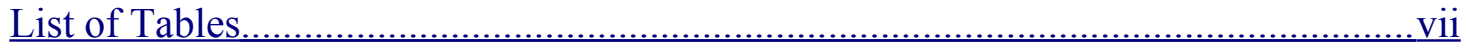

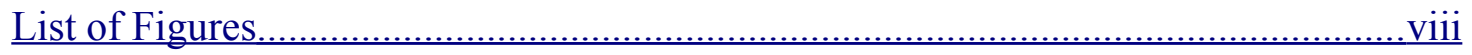

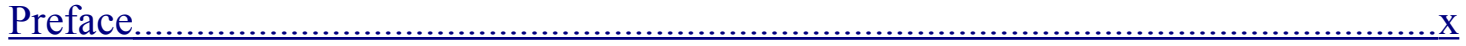

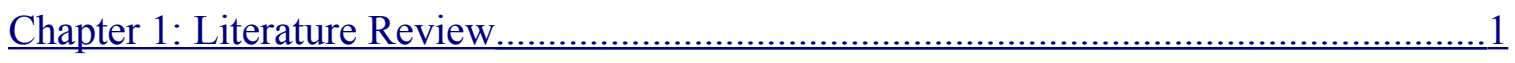

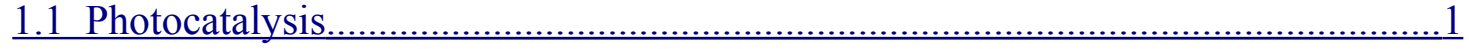

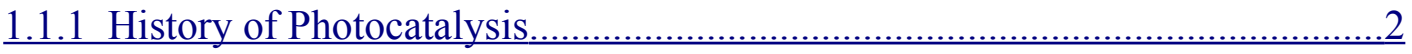

1.1.2 Mechanism for Water Purification by Photocatalysis.............................. 4

1.1.3 Photocatalysis for Water Purification................................................

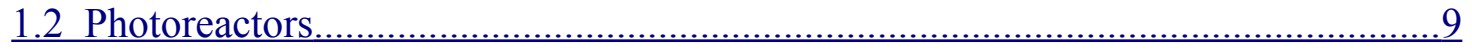

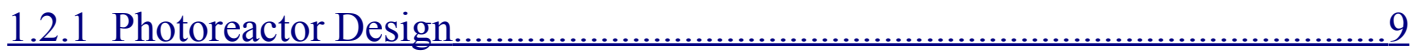

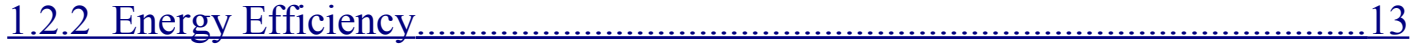

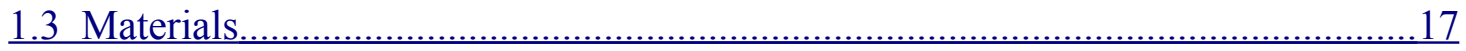

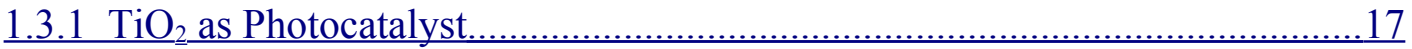

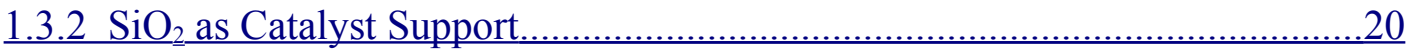

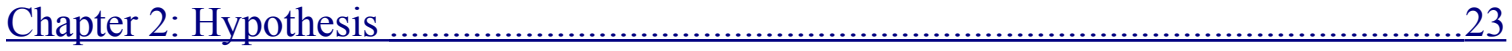

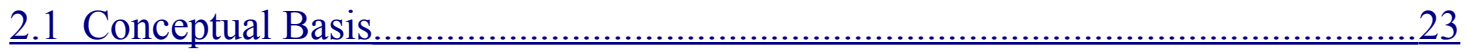

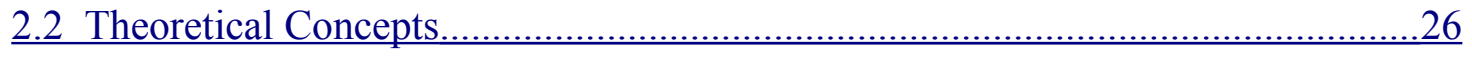

2.3 Defining the Probability Functions.......................................................... 30

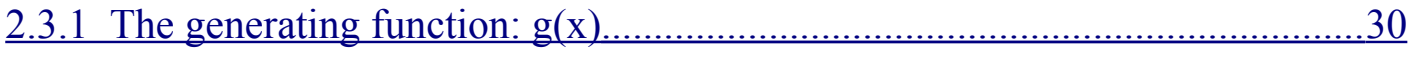

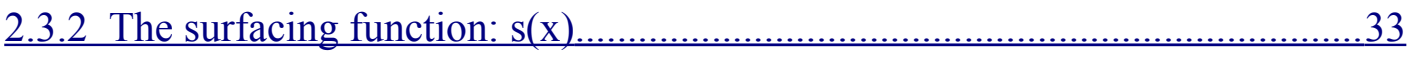

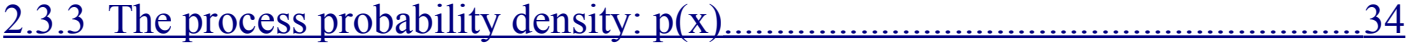

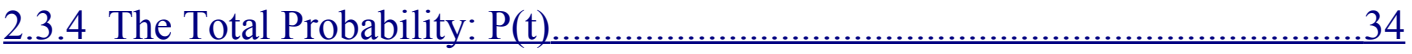




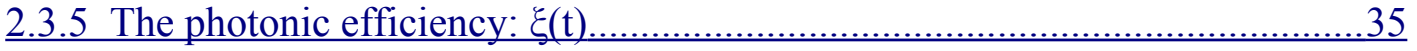

2.4 Support-Side Illumination...............................................................................36

2.5 Multi-Layer Systems............................................................................................40

2.5.1 System Descriptions.....................................................................................40

2.5.2 Calculations of the Photonic Efficiency …………………………..................43

Chapter 3: Photoreactor Design Principles.......................................................................45

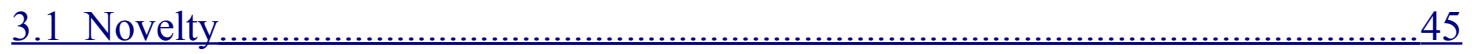

3.2 Description of Photoreactor System.................................................................47

3.3 Photocatalyst Cartridge .....................................................................................49

3.4 Symmetry Alignment with Illumination System.....................................................51

3.5 Light Path Optimization..........................................................................................54

3.6 Application of the Theoretical Model......................................................................56

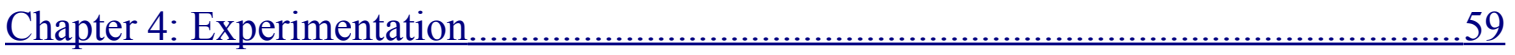

4.1 Photocatalyst Thin-Film Synthesis.............................................................................59

4.1.1 Preliminary Results...........................................................................59

4.1.2 Sol-Gel Dip Coating Method......................................................................61

4.1.3 Qualitative Assessment of $\mathrm{TiO}_{2}$ Films........................................................63

4.1.4 Nanoparticle Implantation........................................................................66

4.1.5 Material Characterization............................................................................68

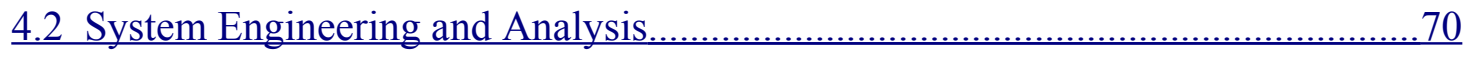

4.2.1 Photoreactor Engineering........................................................................... 71

4.2.1.1 Catalyst Cartridge Fabrication............................................................

4.2.1.2 Illumination System............................................................................. 73

4.2.1.3 Auxiliary Systems.................................................................................

4.2.2 The Analytical System..................................................................................77

4.2.2.1 UV Flowcell Spectrometer................................................................. 77

4.2.2.2 Choice of Contaminants for Degradation Analysis.................................79

4.2.2.3 UVFS Calibration................................................................................. 81

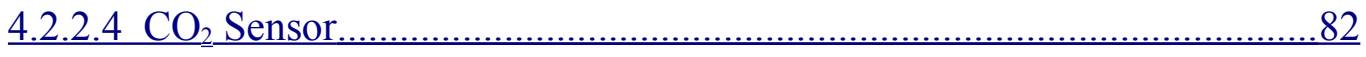




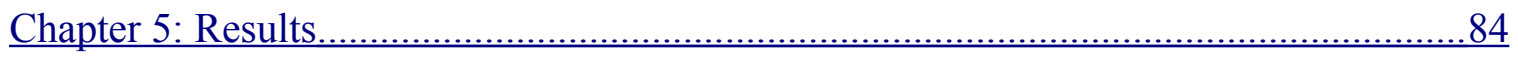

5.1 Photocatalyst Optimization.................................................................................. 84

5.1.1 Thickness Analysis by Optical and Scanning Electron Microscopy .............84

5.1.2 Activity Optimization........................................................................... 87

5.2 Photoreactor Efficiency ....................................................................................93

5.2.1 Prerequisite Measurements........................................................................ 93

5.2.1.1 First-Order Reaction Rate Confirmation.................................................93

5.2.1.2 Oxidation Confirmation..................................................................94

5.2.2 Characterization of Continuous Flow Degradation Behavior.......................... 95

5.2.3 System Optimization...............................................................................97

5.2.3.1 Photocatalyst Film Composition...……………………………………......97

5.2.3.2 Illumination Intensity...........................................................................98

5.2.4 Relationship of EEO to Fundamental Efficiency Parameters.......................100

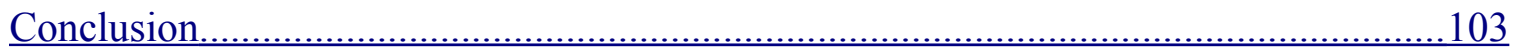

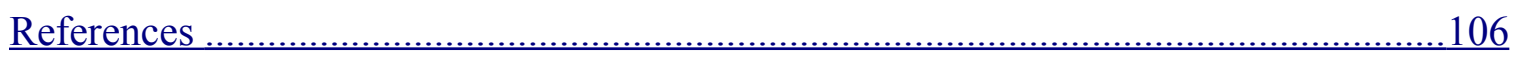




\section{List of Tables}

Table 1: Photocatalytic Reactor Comparison by Electrical Energy per Order.................16

Table 2: Definitions for Symbols and Functions used in Theoretical Model...................30

Table 3: List of Efficiency and Scalability Aspects for Design of 3D Material..............45

Table 4: Synthesis Parameters and Photoactivity Comparison for $\mathrm{TiO}_{2}$ Films................90 


\section{List of Figures}

Figure 1: Typical Reactor Design Styles ........................................................... 10

Figure 2: Energy Band Alignment at the $\mathrm{TiO}_{2}-\mathrm{Water}$ Interface ...................................18

Figure 3: Crystalline Structure Diagrams of Anatase and Rutile $\mathrm{TiO}_{2}$........................19

Figure 4: Geometry and Spatial Variables for Theoretical Model ..............................27

Figure 5: UV Attenuation Plot for Anatase $\mathrm{TiO}_{2}$ for $365 \mathrm{~nm}$ Radiation .........................32

Figure 6: Plot Photodegradation Rate vs Film Thickness for $\mathrm{TiO}_{2}$ Film ......................36

Figure 7: Modified Theoretical Model for Support-Side Illumination ...........................37

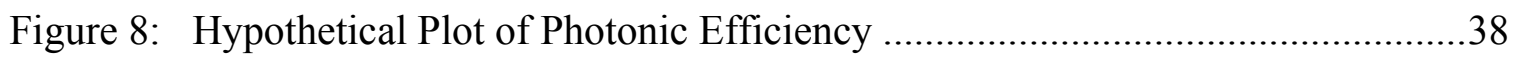

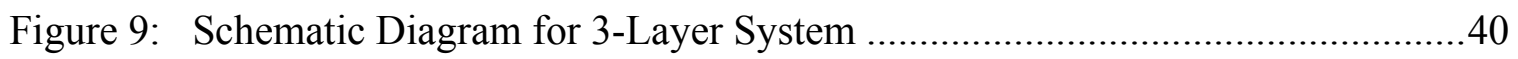

Figure 10: Schematic Diagram for 13-Layer System ................................................ 42

Figure 11: Hypothetical Photonic Efficiency Plots for 3 and 13 Layer Systems ...........43

Figure 12: Photoreactor Design Overview ........................................................ 46

Figure 13: Illumination System Overview ….......................................................48

Figure 14: 3D Model of Photocatalyst Cartridge.......................................................49

Figure 15: Catalyst-Illumination Symmetrical Correspondence ..................................52

Figure 16: Schematic Description of Primary Rods ................................................53

Figure 17: 3D Rendering of Catalyst Structure from LED Perspective ........................55

Figure 18: Example of Optical Hole in Catalyst Material ..........................................55

Figure 19: Adaptation of Theoretical Model to Cylindrical Geometry ..........................57

Figure 20: SEM Images of P25 Immobilization Coatings ..........................................60

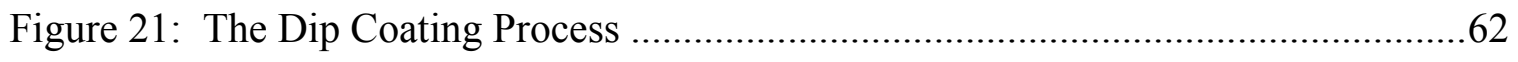

Figure 22: SEM Images of Sol-Gel Derived Films .................................................63

Figure 23: Compositional Adjustment of Sol-Gel Solutions ......................................64

Figure 24: Cracking of Sol-Gel Derived Thin-Films .............................................65

Figure 25: SEM Images of Film Blemishes Caused by Surface Impurities ..................65 
Figure 26: Optical Images of $\mathrm{TiO}_{2}$ Films with Increasing Concentration of P25 ...........66

Figure 27: High-Magnification Images of P25 and $\mathrm{SiO}_{2}$ Modified Films ......................67

Figure 28: TEM Images of Sol-Gel Derived Films Showing Polycrystalline Structure . .68

Figure 29: Raman and UV-Vis Spectra for Sol-Gel Derived Films .............................69

Figure 30: Effects of Heat Treatment on $\mathrm{TiO}_{2}$ Grain-Size and Band-Gap .......................70

Figure 31: Breakdown of Photoreactor System and Analysis Components ....................71

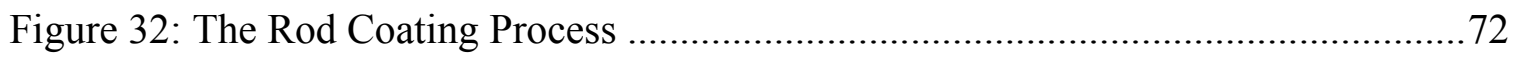

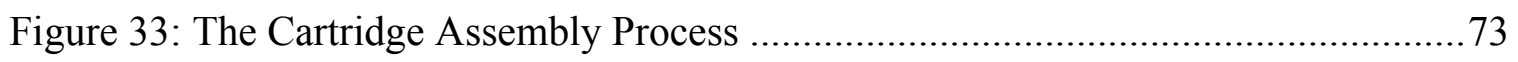

Figure 34: Compatibility of LED Emission Spectrum and Film Absorbance Spectrum ..74

Figure 35: Images of Photoreactor Chamber and Cartridge Insertion ...........................75

Figure 36: Images of Auxiliary System Components ............................................... 76

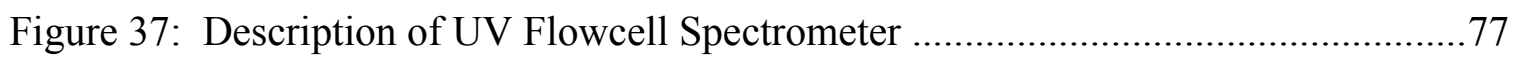

Figure 38: Process Schematic for Evaluation of System EEO …...............................78

Figure 39: Chemical Structure of Chosen Contaminants ......................................... 79

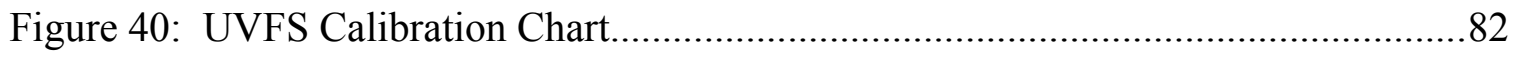

Figure 41: SEM Used for Coating Thickness Analysis ............................................. 84

Figure 42: Analysis of Thickness Control Experiment via UV-Vis Spectroscopy .........85

Figure 43: Correlation of Sol-Gel Viscosity with UV-Vis Absorbance Data ................85

Figure 44: Experimental Set-Up for Film Activity Analysis .....................................87

Figure 45: Film Activity Analysis for Baseline Dipping Solution ..............................8

Figure 46: Degradation Comparison Plots for Thickness and Implantation Trials .........91

Figure 47: Degradation Comparison Plots for Thickness and Implantation Trials ........93

Figure 48: Results for the Oxidation Confirmation Experiment ................................94

Figure 49: Characterization of Reactor Data for Continuous-Flow Operation ...............95

Figure 50: Switch-On Event Characterization Event and Cartridge Comparison ..........97

Figure 51: Results for Illumination Intensity Optimization Experiment .......................98 


\section{Preface}

The following manuscript presents a scientific investigation into the application of semiconductor photocatalysis for the purpose of water purification. The focus of this research was the improvement of system efficiency through the development of a 3D photocatalyst material that occupies the volume of a photoreaction chamber. As this work was done for fulfillment of a degree in Applied Physics, it is particularly aimed at addressing the material properties of the photocatalyst and the photonic efficiency of the system, while also ensuring that results are applicable to practical and scalable systems.

Although photocatalysis has been very successful at degrading aqueous organic materials, the photocatalytic process has proven to be very difficult to implement. Therefore, it was determined that the greatest contribution to the field could be made by addressing the primary limitations of process implementation. Two primary challenges were identified as obstructions to practical use of photocatalysis for water purification: 1) expensive operational costs, i.e. energy usage, components of illumination system, material fabrication, and catalyst replacement; 2) low energy efficiency of the overall process, due to material limitations and the complexity of seemingly contradictory design requirements. The goal of this work then is to address both of these issues through the development of a novel 3D photocatalyst material in combination with a geometrically corresponding photoreactor, which are designed to work together, systematically, to achieve a high energy efficiency for the reaction.

The first chapter reviews the fundamental research that this work is based upon. This includes a survey of the field of photocatalysis, an investigation into various methods of 
implementation and their limitations, as well as an assessment of the materials chosen for this particular study.

In the second chapter, a theoretical model is presented, which investigates the influence of the optical and electronic properties of the 3D photocatalyst material on the energy efficiency of a photocatalytic reactor. This model is then used to generate a hypothesis for improving the design of photocatalysis based on existing water purification reactors.

The implications of this model are then considered in light of the conclusions drawn from the review of photoreactor limitations in Chapter 1. The result is the design of a 3D material and a corresponding UV illumination system that is presented in Chapter 3. This design incorporates aspects of efficiency, practicality, and scalability to create a novel photocatalytic water purification system that is capable of operating in industrial settings.

The engineering aspects of developing this system are then described in Chapter 4, along with a presentation of the chemical synthesis and material analysis techniques that were used to fabricate an effective 3D photocatalyst material. This chapter then goes on to provide a description of the analysis system developed for evaluating the system efficiency.

The scientific results are then presented and discussed in Chapter 5. This includes experiments done to synthesize and characterize the photocatalyst material, as well as photoreactor degradation data, which was used to calculate pollutant removal efficiency for the custom built photoreactor system. 
Finally, a conclusion section summarizes the results of this investigation and elucidates the contribution of this work to the field of photocatalysis. This is followed by a brief description of the intentions for the future works of this project. 


\section{Chapter 1: Literature Review}

\subsection{Photocatalysis}

In essence, photocatalysis is the absorption of light and the conversion of the absorbed energy into a chemical form through the use of semiconductor material. A semiconductor material is capable of absorbing a photon and using its energy to convert a bound electron into a free electron by excitation from the valence band to the conduction band. In order for this transition to occur, the photon must have an energy that is greater than the band gap of the material. For example, the band gap energy for $\mathrm{TiO}_{2}$ photocatalysts is approximately $3.2 \mathrm{eV}$ depending upon the particular crystallinity, size, and fabrication method of the material at hand [1]. This corresponds to a photon wavelength of $387 \mathrm{~nm}$; thus, only light with a wavelength smaller than this is capable of initiating a photocatalytic reaction.

If the photon does have equal or greater energy than the material band gap, then two charge carriers can be created upon absorption of the photon: a negatively charged electron $\left(e^{-}\right)$and a positively charged hole $\left(h^{+}\right)$. Both of these carriers are able to move by diffusion throughout the semiconductor until they find a suitable reaction site. If an electron and hole encounter one another they may interact in an appropriately named process called recombination. This is essentially a complete loss of the initially absorbed photon energy, and thus a reduction of process efficiency. However, if the charge carriers are able to reach the surface of the semiconductor, they will be able to react with 
whatever substance may be present at the semiconductor interface. Since the process at hand is water purification, the substance at the interface is predominantly water.

The essential reaction of the process is initiated when $h^{+}$reacts with the water to form reactive oxygen species (ROS). These highly electronegative molecules then degrade contaminants dissolved in the water through a series of redox reactions. Because photocatalysis is capable of generating hydroxyl radical $(\mathrm{OH} \bullet)$ molecules, which have the highest redox potential of all ROS molecules, it is categorized an Advanced Oxidation Process (AOP). AOPs are highly regarded for their ability to completely mineralize organic molecules. Over the past 4 decades, there have been numerous studies that have shown that photocatalysis is capable of removing Total Organic Carbon (TOC) from water through successive oxidation and reduction of substances that are suspended or dissolved in water.

\subsubsection{History of Photocatalysis}

The phenomenon of photocatalysis is often cited as being discovered by Akira Fujishima in 1972 with his seminal paper "Electrochemical Photolysis of Water at a Semiconductor Electrode" [2]. However, this is untrue as even Fujishima himself asserts that his work was fundamentally based off of many earlier discoveries dating back to an observation by Renz in 1921 that sunlight shining on a titania surface, in the presence of organic compounds, caused a chemical reaction to occur [3]. It was soon realized that similar reactions involving another wide bandgap semiconductor, $\mathrm{ZnO}$, were the result of simultaneous oxidation and reduction reactions [4]. 
This ultimately led to a fundamental understanding of a phenomenon previously observed by painters, that $\mathrm{TiO}_{2}$ based paints obtained a chalky film on the surface if left in sunlight [5]. In light of the new scientific understanding proposed by Renz, Baur and Perret, it was understood that this effect was caused by the degradation of the paint's organic solvent by the photochemical reaction induced by the interaction of sunlight and $\mathrm{TiO}_{2}$. This knowledge led to the first experiment that resembled modern photocatalysis studies, conducted by Goodeve and Kitchener in 1938, in which an organic dye was degraded by a titania powder, and included an evaluation of the reaction's photonic quantum yield [6].

Continuation of this type of experimentation continued to develop these ideas, including the investigation of various other semiconductor materials, as well as speculation about the reaction products, reactants, and pathways. The focus began to shift away from $\mathrm{TiO}_{2}$ based photocatalysts to materials such as $\mathrm{ZnO}$ and $\mathrm{CdS}$, which showed higher photoactivity [7]. However, the lack of photostability of these materials prompted a return of focus to $\mathrm{TiO}_{2}$ by the 1960 's.

At this point researchers began studying the differences between the various phases of $\mathrm{TiO}_{2}$, coming to the now well accepted conclusion that the anatase phase exhibits the highest photocatalytic activity [8]. The following year, the first complete conversion of organic substrates (i.e. ethylene and propylene) into $\mathrm{H}_{2} \mathrm{O}$ and $\mathrm{CO}_{2}$ was observed [9].

However, it wasn't until the Nature publication by Fujishima in 1972 that the field of photocatalysis began to gain significant attention. In this article, it was reported that $\mathrm{TiO}_{2}$ photocatalysis could produce hydrogen gas; the idea of using this process as a form of solar energy conversion quickly took hold. From that point, the number of proposed 
applications for photocatalysis began to expand dramatically. Of particular importance to this research, Frank and Bard proposed the application of photocatalysis for the purpose of water purification in 1977 [10]. This led to an explosion of studies carried out over the subsequent decades to determine the scope, viability, and intricacies of this idea. In the 2 decades that followed, the focus turned to investigating the use of photocatalysis for the destruction of various aqueous and airborne pollutants, along with hypothesizing about the mechanisms responsible for these phenomena.

\subsubsection{Mechanism for Water Purification by Photocatalysis}

The photo-induced redox process that leads to the destruction of organic pollutants in water are simplified below for a generic organic contaminant, R. The exact reaction pathways are extremely varied depending upon the molecular properties of the contaminant and chemical constitution of the aqueous solution. Nevertheless, all

photocatalytic reactions involve four fundamental reactions: 1) the absorption of a photon $(\gamma)$ by the photocatalyst $\left(\mathrm{TiO}_{2}\right)$ to produce electron-hole pairs; 2$)$ oxidation of a water molecule by $\mathrm{h}+$ at the catalyst-water interface to form $\mathrm{OH} \bullet$; 3) reduction of dissolved oxygen by e- at the catalyst-water interface to form superoxide (O2-); 4) direct oxidation of the pollutant adsorbed (Rads) onto the catalyst surface [11].

$$
\begin{aligned}
& \gamma+\mathrm{TiO}_{2}=\mathrm{TiO}_{2}+e^{-}+h^{+} \\
& h^{+}+\mathrm{H}_{2} \mathrm{O}=\mathrm{OH}^{\bullet}+\mathrm{H}^{+} \\
& e^{-}+\mathrm{O}_{2}=\mathrm{O}_{2}^{-}
\end{aligned}
$$




$$
h^{+}+\mathrm{R}_{a d s}=\mathrm{R}^{+}
$$

These four reactions, though simplified, are used to emphasize two key points about the photocatalytic degradation processes that will be important in a later discussion. First, that both photogenerated carriers, $e^{-}$and $h^{+}$, are necessary reactants for complete degradation of pollutants to occur. Although the $h^{+}$species is generally regarded as being responsible for the degradation, if the $e^{-}$species are not removed from the photocatalyst material, then a net charge will accumulate, which ultimately quenches the reaction. Thus, it is important to make sure there is enough dissolved oxygen in the solution to allow for electrons to be removed at the same rate as the holes. Second, there are two basic pathways for oxidation of pollutants to occur; that is, the pollutant can either be oxidized by the catalyst material directly or through the generation of $\mathrm{OH} \bullet$ species. It is generally accepted that the latter pathway is primarily responsible for the occurrence of degradation $[2,11]$. However, it is also often reported that molecules with higher affinity for the photocatalyst surface are correlated with higher degradation rates [12-13]. This suggests that direct degradation of the pollutant molecules is also a contributing factor. More rigorous descriptions of the reactions taking place at the photocatalyst surface are provided in many survey articles focusing on this effect [3, 14-18]. One particularly detailed scheme was presented by Herrmann [19] and takes the transient radicals into account. The dot $(\bullet)$ next to a molecular symbol represents an unpaired electron, signifying the designation of the molecule as a radical. Radicals are charge neutral but highly reactive, due to their strong tendency to form bonds. 


$$
\begin{aligned}
& \gamma+\mathrm{TiO}_{2} \rightarrow \mathrm{TiO}_{2}+e^{-}+h^{+} \\
& e^{-}+\mathrm{O}_{2} \rightarrow \mathrm{O}_{2}^{-} \\
& \mathrm{H}_{2} \mathrm{O} \rightleftharpoons \mathrm{OH}^{-}+\mathrm{H}^{+} \\
& \mathrm{OH}^{-}+\mathrm{h}^{+} \rightarrow \mathrm{OH}^{\bullet} \\
& \mathrm{O}_{2}^{-}+\mathrm{H}^{+} \rightarrow \mathrm{HOO}^{\bullet} \\
& 2 \mathrm{HOO}^{\bullet} \rightarrow \mathrm{O}_{2}+\mathrm{H}_{2} \mathrm{O}_{2} \\
& \mathrm{H}_{2} \mathrm{O}_{2} \rightarrow 2 \mathrm{OH} \\
& \mathrm{H}_{2} \mathrm{O}_{2}+e^{-} \rightarrow \mathrm{OH}^{\bullet}+\mathrm{OH}^{-} \\
& \mathrm{R}-\mathrm{H}+\bullet \mathrm{OH}^{\bullet}+\mathrm{R}^{\bullet} \mathrm{O} \\
& \mathrm{R}-\mathrm{OH}+\bullet \mathrm{OH}^{-} \mathrm{Intermediates}^{-} \mathrm{CO}_{2} \\
& \mathrm{R}^{*}-\mathrm{COOH} \rightarrow \mathrm{R}^{*}-\mathrm{H}^{-} \mathrm{CO}_{2}
\end{aligned}
$$

These reactions enable organic compounds to be broken down by successive interactions with the hydroxyl radicals, known as photo-Kolbe reactions (Equation 15), leading to complete mineralization of the polluting molecules. If this process is allowed to proceed to completion the only end products are $\mathrm{CO}_{2}, \mathrm{H}_{2} \mathrm{O}$, and simple mineral acids $[14-15,20]$. 
This is one of the most attractive features of AOPs for environmental pollutant remediation.

The fact that hydroxyl radicals are 1) generated in this reaction, and 2) are primarily responsible for the degradation of pollutants in the water purification process allows for this process to be generally classified as an Advanced Oxidation Process (AOP). There is a great deal of research on the subject of pollutant degradation by AOPs, including reaction rates and pathways for various environmental pollutants [21-24]. Of particular importance for this research is the fact that AOPs have been shown to be non-preferential in their destruction of pollutants. This means that although reaction rates vary from one pollutant to the next, nearly all organic material can be mineralized due to the extremely high redox potential of the hydroxyl radical, $+2.85 \mathrm{~V}$ relative to a Standard Hydrogen Electrode (SHE) [14].

\subsubsection{Photocatalysis for Water Purification}

Human activity inevitably leads to contamination of the earth's water systems with a vast array of anthropogenic organic molecules. Current municipal systems are designed to clean wastewater so that it can be returned to the environment without causing significant alteration to the municipality's immediate surroundings. However, this cleaning process is typically not effective enough to allow this effluent to be used for potable, or even nonpotable applications because there are many contaminants that are not completely removed from the water during standard treatments. Therefore, the effluent is then typically discarded to rivers and lakes [25]. 
These pollutants often end up in our drinking water because the source water for drinking water treatment plants is contaminated by the effluent from wastewater treatment facilities. In 1980, an EPA study determined that at one municipal drinking water facility, $16 \%$ of the source water was actually effluent from a nearby wastewater facility [26]. A more recent study determined that between 1980 and 2008 the problem has been dramatically exacerbated due to an average increase in municipal effluent output by $68 \%$ over this time period [27]. When these organic molecules are treated by drinking water facilities, they have a high potential to produce chlorine disinfection byproducts.

A study conducted by the Environmental Working Group, a non-profit water quality research group, conducted an analysis on almost 20 million records obtained from state water officials and found 316 pollutants in the drinking water from 45 states [28]. Only about one-third of the chemicals they found in our water supply are regulated by the EPA for health and environmental impact, despite the fact that health standards have been set for many of these unregulated chemicals [29]. The majority of these unregulated chemicals are organic molecules introduced into the water supply system from pesticides, pharmaceuticals, industrial byproducts, and urban runoff.

Thus, there is a drastically increasing need for processes that can remove organic molecules from our wastewaters in an effective and efficient manner. Water treated with UV light is effective for killing pathogens through inactivation of the organism's ability to reproduce and is one of the four methods of disinfection approved by the United States FDA [30]. Large-scale, municipal and industrial UV treatment systems are currently used for disinfection purposes. However, these systems are not currently used for water 
purification. It is possible to use UV light to degrade organic molecules, to some extent, through photolysis; however, this process is quite inefficient and is ineffective for molecules that do not absorb UV light [30].

However, it may be possible to enhance the capability of these existing systems by incorporating photocatalytic materials into the reaction chamber of UV treatment systems. A recent survey of the various pollutants that have been tested for photodegradation by nanocrystalline $\mathrm{TiO}_{2}(\mathrm{NTO})$ was compiled by Lazar et al., who concluded that "The spectrum of compounds that are susceptible to the destructive power of [nanocrystalline titanium dioxide] photocatalysis is remarkable, comprising families of dyes, pesticides, herbicides, pharmaceuticals, cosmetics, phenolic compounds, toxins, and more" [11].

Evidence in support of the generalized effectiveness of this process has recently led to a transfer of focus from the fundamental chemical processes that occur as a result of the hydroxyl radical interactions to the efficiency of hydroxyl radical generation by the system. Therefore, research on photocatalysis of the past decade has been focused on the improvement of photoreactor designs, with the intention of making the process more applicable to industrial demands.

\subsection{Photoreactors}

\subsubsection{Photoreactor Design}

In general, water purification by photocatalysis is accomplished through the use of a photoreactor, which has three necessary parts: 1) a reaction chamber, 2) an illumination system, and 3) a hydraulic system. The way that these 3 parts are utilized comprises the 
design of the photoreactor. Ultimately, the goal is to degrade the organic material in the liquid as fast as possible and with the highest energy efficiency.
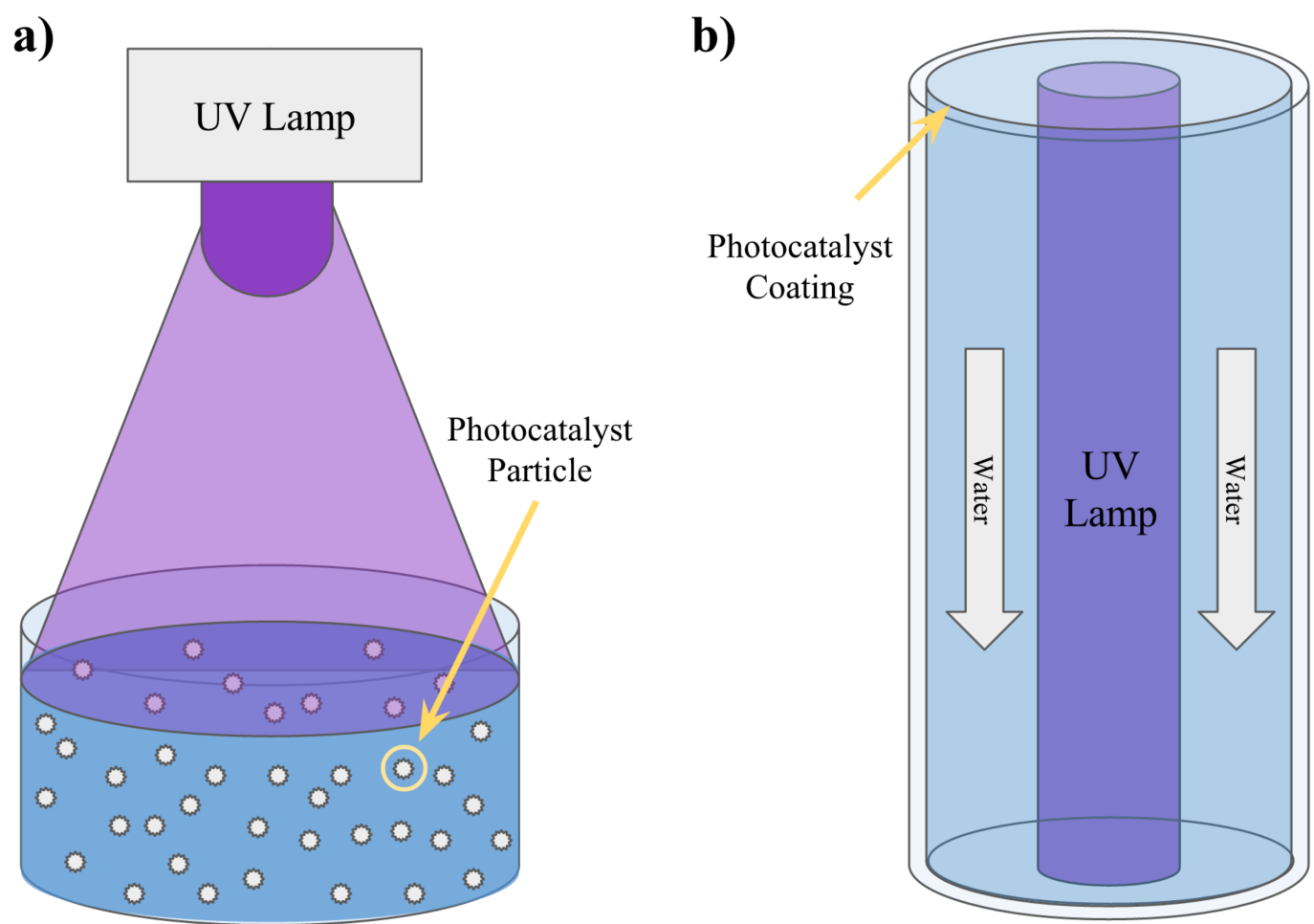

Figure 1 - Typical Reactor Design Styles: a) a simple example of a slurry reactor, consisting of a suspension of photocatalyst particles that is illuminated with UV light (typically mixed using magnetic stir bar); b) example of a fixed photocatalyst flowreactor, consisting of a UV lamp encased at the center of a tubular reaction chamber that has been coated with a photocatalyst film.

However, there is a trade-off between these two objectives because the highest process efficiency occurs at low illumination intensity, yet higher degradation rates can be achieved at a higher intensity but with the cost of more wasted energy. This has led to a great deal of uncertainty within the photocatalysis field because systems that display fast degradation of contaminants are found to be cost prohibitive, while highly efficient systems do not process water at a fast enough rate to be considered practical. Thus, attempts to design an effective photoreactor have separated into two distinct categories: 
those that use a fixed photocatalyst and those that use a particle suspension. An example of each type of reactor is shown in Figure 1.

Those that choose to use the particle suspensions do so because this method has the highest surface area to volume ratio of the photocatalyst material and mass transfer limitations are avoided. However, this method also requires the removal of the particles before the water can be considered purified. This is a non-trivial issue, but has been accomplished - primarily by means of flocculation, centrifugation, magnetophoretic separation, or filtration. Yet in each case system complexity is increased and additional energy is spent on this process. Furthermore, the lifetime of these particles is short; thus, they must be replenished frequently. Two examples of well-designed systems are the Plataforma Solar de Almería - a massive array of parabolic trough reactors in Spain and the Purifics Photo-Cat - an industrially sized purification system. Although these systems have been shown to be very effective at pollutant removal, they have not yet claimed market share due to high system operation costs [31].

On the other hand, systems that utilize a fixed catalyst do so with the intention of simplifying the overall process by allowing the contaminated water to flow across the surface of a rigid photocatalyst material. This prevents complications with particle removal but also greatly reduces both photocatalyst surface area and reaction quantum yield - leading to lowered energy efficiency of the purification process.

Within the fixed catalyst classification there are two subclasses. The first contains those that fabricate $2 \mathrm{D}$ photocatalyst membranes that can be used in a way similar to the use of a filter. The most effective materials in this category are porous sheets of photocatalyst 
material that force the contaminated water to pass through the photocatalyst. However, photoreactors that attempt to use these photocatalytic membranes can only operate at very low pressures due to low strength of the materials, which have led to severe limitation of flow rate. Some 2D photocatalysts have seen success as antibiological coatings for filter media, but have not been shown to be scalable for the purpose of water purification.

The second subclass of fixed photocatalysts is that of 3D photocatalyst materials. This involves the creation of a photocatalyst material that fills the 3-dimensional volume of the reaction chamber. Systems of this type can have high surface area, do not require catalyst particle separation, and can be made with high strength and durability. However, these systems are also complicated by the fact they are highly dependent on optical properties of the reaction chamber, the photocatalyst material itself, and the illumination system.

Additionally, the chemical kinetics must be considered when designing the 3D material since mass transfer of pollutants to the photocatalyst surface can be severely limited if the catalyst surface area to water volume ratio is too low. For example, the design shown in Figure $1 \mathrm{~b}$ utilizes a coating of photocatalyst material on the interior surface of a cylindrical tube [32]. In order to obtain a high surface area to water volume ratio, the radius of the tube must be decreased to the point that high flow rates are impossible.

At the opposite extreme, many researchers will coat small but macroscopic substrates such as glass beads [33], glass rings [34], or quartz fiber [35] - with a photocatalyst material and pack the substrates into the reaction chamber so that the water is forced to percolate through the chamber. This approach has the capability to solve the mass 
transfer limitations, but is rather haphazard with regard to the optical considerations. Therefore, it is necessary to use a standard method of comparison in order to determine which designs are the most effective.

\subsubsection{Energy Efficiency}

In order to evaluate the effectiveness of photoreactor design, it is necessary to compare the energy efficiency of a system to that of other photoreactor designs. However, there has been much debate about how exactly to define and report efficiency values for photocatalytic reactions. This complication seems to have arisen due to the differences between the fields of heterogeneous catalysis and homogeneous catalysis.

Homogeneous catalysis, which is defined as a catalytic reaction in which the phase of the catalyst is the same as that of the reactants, was already an established and well-defined field of study by the time that heterogeneous catalysis was emerging. In particular, the terms quantum yield and quantum efficiency had been defined without the need to account for scattering and reflection of light at the catalyst-reactant interface. However, in the heterogeneous case, where the catalyst is in the solid phase, these interactions need to be accounted for because of the difference in the indices of refraction between the solid catalyst and the liquid reactants [36].

This led to the creation of various new terminology such as apparent quantum yield and photonic efficiency, which are very useful but have not been used with consistency. Eventually, some agreement was established when the figure-of-merit technique put forth by Bolton et al., which was accepted as the standard method of comparison for advanced oxidation processes by the International Union of Pure and Applied Chemistry (IUPAC) 
[37]. For pollutants of low concentration $(<100 \mathrm{ppm})$, as is the case in this study, this involves the computation of the Electrical Energy per Order (EEO), which can be used to quantify the electrical energy required to degrade the aqueous TOC by one order of magnitude.

The system EEO has been formulated in two ways. The first is in terms the initial and final concentrations of pollutant $\left(c_{i}\right.$ and $\left.c_{f}\right)$, the electrical power provided to the illumination system $(P)$, and the system flow rate $(F)$. The second is in terms of the three efficiency parameters - the absolute photon flow $(G)$, the optical yield $(\chi)$ and the material quantum yield $(\phi)$ - as well as reaction rates for degradation of the contaminant $\left(k_{C}\right)$, the reaction rate for interactions with scavenger molecules $\left(k_{s}\right)$, and their respective concentrations $(S)$ [37]. These are given as Equation (16) and Equation (17), respectively.

$$
\begin{aligned}
& E E O=\frac{P}{F \log _{10}\left(c_{i} / c_{f}\right)} \\
& E E O=\frac{0.640 P \sum k_{s_{i}}\left[S_{i}\right]}{G \chi \phi k_{C}}
\end{aligned}
$$

The former is the most practical form, as it can be calculated in a relatively straightforward way if the concentration of contaminant can be measured at the reactor outlet. This capability also enables time series measurements to be collected, allowing for calculation of the degradation rate constant and verification of overall 1 st order reaction kinetics, which is required to validate the assumptions used to derive Equation (16) and Equation (17). On the other hand, the latter equation is useful for understanding the EEO 
values theoretically, as it mathematically connects the system energy usage to the fundamental efficiency parameters of the degradation process.

However, despite the acceptance of this method by the IUPAC, the majority of publications on photocatalytic reactors still do not report EEO values. It is unclear if this is due to the fact that most authors are unaware of this attempt at standardization, if they don't have analytical capability to make the proper measurements, or perhaps they don't want to report their value due to extremely high energy use by the system. This observation was also made by Marugán et al. in an article on supported photocatalyst materials in the journal Water Research [38]:

"Despite the large number of supported $\mathrm{TiO}_{2}$ photocatalysts reported in the last years, few attempts have been made to evaluate the optical behavior of these materials in terms of photonic efficiencies or quantum yields, optical parameters standardized by the IUPAC. It is important to note that these parameters are absolutely required for the rigorous design and scaling-up of photocatalytic reactors in order to accomplish a commercial application of photocatalytic technologies."

Nevertheless, there have been a few studies done on comparable systems that have reported EEO values and are presented in Table 1. This shows a general trend of lower EEO values for particle suspension (slurry) type reactors, mid-range values for immobilized catalysts, and higher values for catalyst films. However, as mentioned previously, the values for slurry reactors are deceiving since EEO includes only energy consumed by the illumination system and ignores the energy required for catalyst particle removal from the effluent. 


\begin{tabular}{|l|c|c|c|l|}
\hline \multicolumn{1}{|c|}{ Author } & Year & EEO & Type & \multicolumn{1}{c|}{ Photocatalyst Material } \\
\hline Li [33] & 2015 & 5200 & Film & $\mathrm{TiO}_{2}$-film on silica gel beads \\
Natarajan [32] & 2011 & 1800 & Film & $\mathrm{TiO}_{2}$-film in quartz tube \\
Ferreira [39] & 2016 & 220 & Slurry & $\mathrm{TiO}_{2}$ slurry \\
Lizama [34] & 2002 & 140 & Immobilized & $\mathrm{TiO}_{2}$ immobilized on glass rings \\
Khataee [12] & 2009 & 85 & Immobilized & $\mathrm{TiO}_{2}$ immobilized on glass plate \\
Fowler & 2017 & 54 & Film & $\mathrm{TiO}_{2}$-film - optimized by $\mathrm{TiO}_{2}$ and $\mathrm{SiO}_{2}$ particles \\
Mansilla [40] & 2006 & 49 & Slurry & $\mathrm{TiO}_{2}$ slurry \\
Benhajady [41] & 2011 & 32 & Slurry & $\mathrm{TiO}_{2}$ slurry \\
Lizama [34] & 2002 & 22 & Slurry & $\mathrm{TiO}_{2}$ slurry - optimized conditions \\
Mansilla [40] & 2006 & 18 & Slurry & $\mathrm{TiO}_{2}$ slurry with added iron particles \\
Lizama [33] & 2002 & 7.6 & Slurry & $\mathrm{ZnO} \mathrm{slurry} \mathrm{-} \mathrm{optimized} \mathrm{conditions}_{\text {Benotti [42] }}^{2009}$ \\
\hline
\end{tabular}

Table 1 - Comparison of EEO $\left(\mathrm{kWh} / \mathrm{m}^{3}\right)$ values for photocatalytic degradation reported in literature. For studies that reported values for multiple contaminants, the average is displayed.

This analysis thus clarifies the objective for creation of a 3D photocatalyst material: to achieve system EEO values comparable to those for slurry type type reactors. This is because slurry type reactors are considered to possess optimal reaction conditions. Therefore, if a photoreactor can be created with 1) a fixed catalyst material that does not require post treatment of the effluent, 2) a durable and regenerable catalyst material, and 3) EEO values comparable with those of slurry reactors, then the field of photocatalysis will be brought closer to the goal of industrialization. A recent statement about the field of photocatalytic water treatment (PWT) summarizes this well: "To preserve the appealing qualities of PWT that triggered the hype to begin with, technologies that accommodate its multidimensional aspects without accumulating excessive ancillary sources of energy consumption are necessary" [31]. 


\subsection{Materials}

The difficulty, elucidated by analysis of Table 1, is that slurry type reactors are efficient but expensive and fixed catalyst reactors are simple but inefficient. Thus, the solution. The aim of this research was to solve this issue by creating a fixed catalyst system that can achieve the efficiency level of slurry reactors. This was done by creating a 3D material with the following properties: 1) reduced energy losses due to parasitic absorption and charge carrier recombination, 2) low fabrication costs, and 3) increased material durability and lifetime. The following sections present a survey of the materials chosen for this research, along with a justification of these choices.

\subsection{1 $\underline{\mathrm{TiO}}_{2}$ as Photocatalyst}

There several important material qualities that lead to the choice of titanium dioxide $\left(\mathrm{TiO}_{2}\right)$ as the ideal testing material. In particular, this material is widely accepted as the most practical photocatalyst known to exist. Although there are a few materials that exhibit higher photocatalytic activity, e.g. $\mathrm{ZnO}$ and $\mathrm{CdSe}[2-3]$, there are many other factors that are equally as important with regard to the application of photocatalysis: photostability, non-toxicity, environmental safety, resistance to chemical corrosion, properly situated valence and conduction band edges, abundance, and cost. With respect to this extremely restrictive list, $\mathrm{TiO}_{2}$ is currently accepted as the best candidate for commercialization and industrialization.

Given the fact that photocatalysis is defined as a process that is mediated by the absorption of light by a semiconductor material, it is thus apparent that the crystallinity of the semiconductor has great influence on the reaction rate and process efficiency. This 
must be so, as it is indeed crystallinity of a material that gives rise to semiconductor behavior.

Indeed, the most fundamental connection between the material properties of $\mathrm{TiO}_{2}$ and its effectiveness as a photocatalyst is the alignment of its conduction and valence band energies with the redox potential requirements for the necessary chemical reactions. This alignment is shown for anatase $\mathrm{TiO}_{2}$ in Figure 2. It is clear from this diagram why $\mathrm{TiO}_{2}$ is so well suited for photocatalysis: first, the reduction potential of a conduction band electron is $-0.52 \mathrm{eV}$, slightly greater than the $-0.28 \mathrm{eV}$ required to reduce diatomic oxygen $\left(\mathrm{O}_{2}\right)$ to superoxide $\left(\mathrm{O}_{2}^{-}\right)$; second, the oxidation potential of a valence band hole is $+2.53 \mathrm{eV}$, slightly greater than the $+2.27 \mathrm{eV}$ required to produce $\mathrm{OH} \bullet$ from $\mathrm{H}_{2} \mathrm{O}$ [2].

\section{Potential vs. Standard Hydrogen Electrode at p.H. 7}

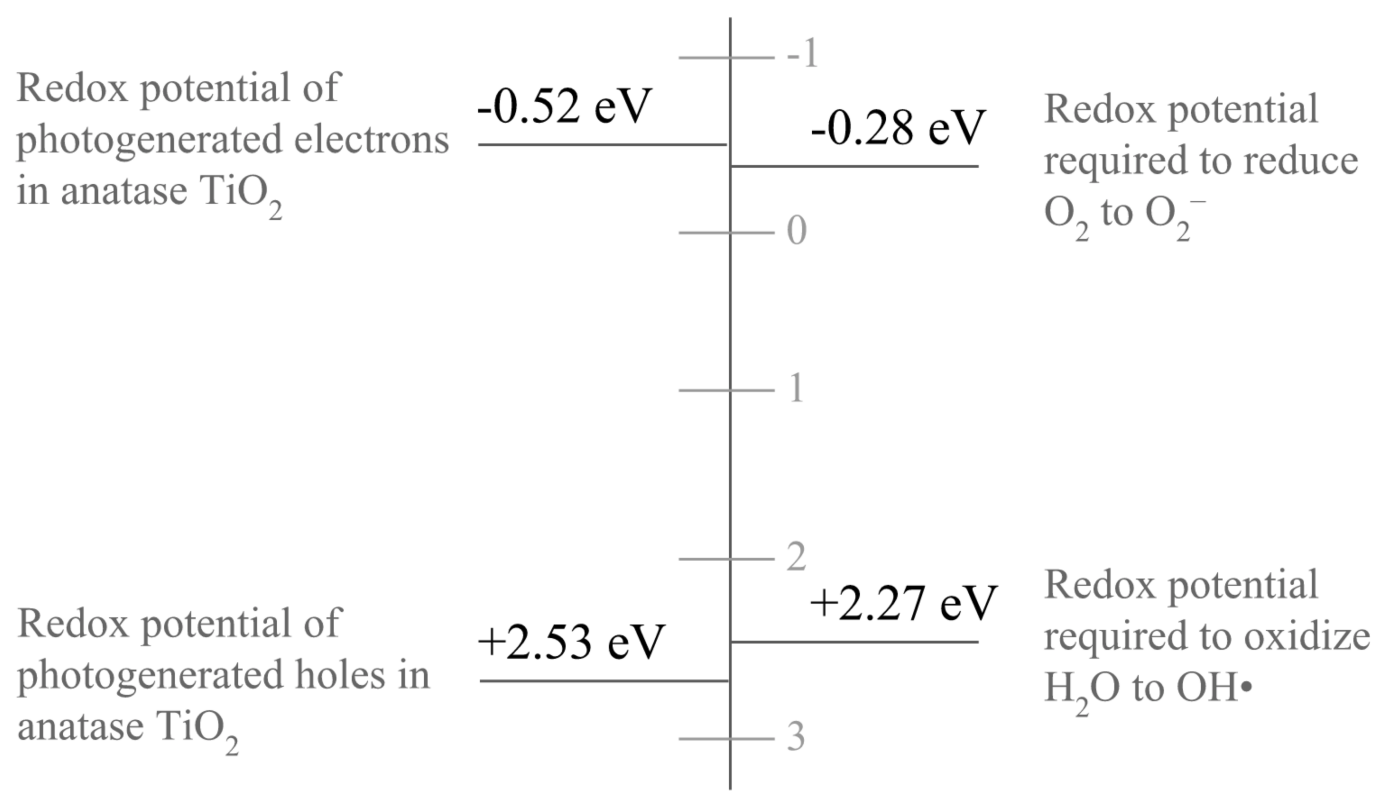

Figure 2: Energy diagram of the $\mathrm{TiO}_{2}$ conduction and valence band edges relative a S.H.E., showing alignment with the redox potentials for the reduction of oxygen and oxidation of $\mathrm{H}_{2} \mathrm{O}$. 
This alignment is the reason that the photocatalytic reaction occurs spontaneously with $\mathrm{TiO}_{2}$ once the charge carriers have been generated. This is also the reason that many efforts to improve the photoactivity of $\mathrm{TiO}_{2}$ by reducing its band gap have failed. If either the conduction band energy is decreased by more than $0.24 \mathrm{eV}$, or the valence band energy is increased by more than $0.26 \mathrm{eV}$, these reactions cannot proceed.

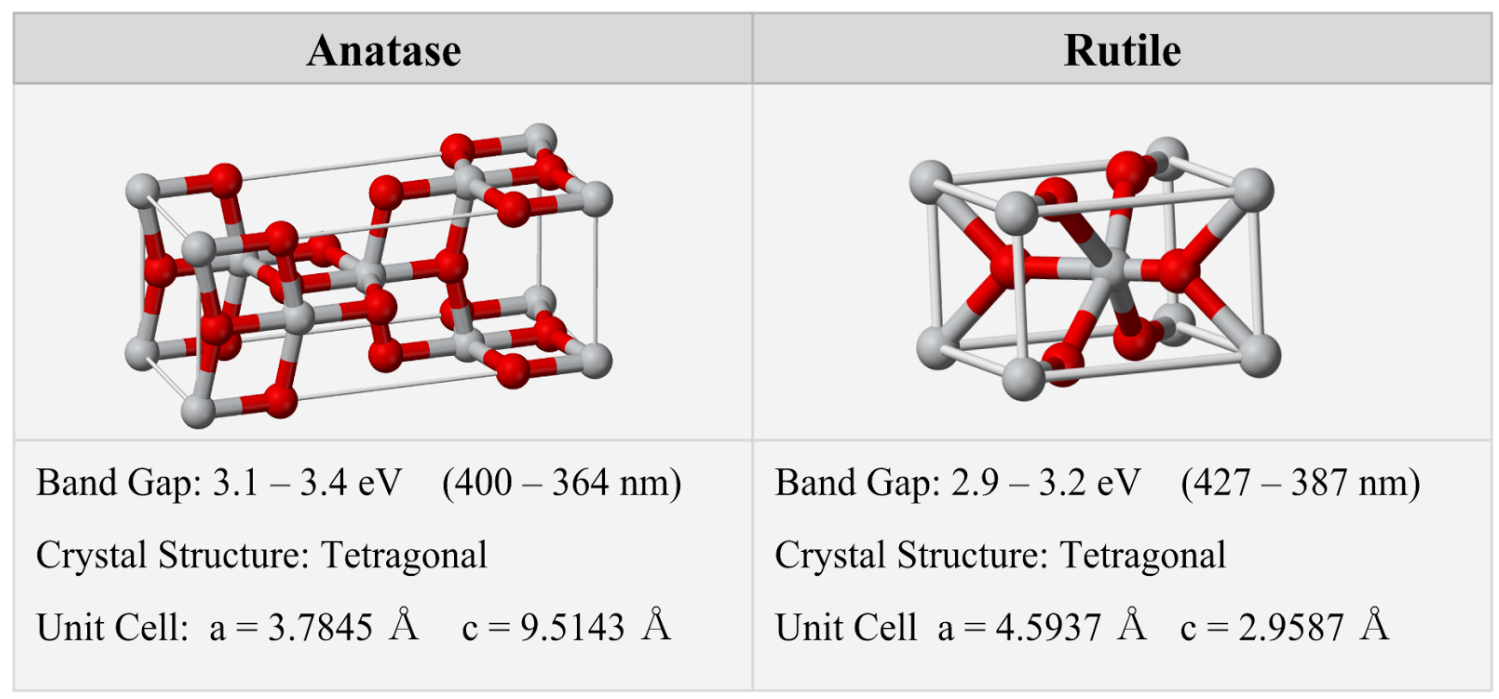

Figure 3: Crystal structure diagrams for the two photocatalytically active phases of $\mathrm{TiO}_{2}$ a) anatase and b) rutile. Although anatase displays higher photoactivity, the highest activity is achieved with mixed-phase materials having an anatase:rutile ratio of $\sim 3: 1$.

The crystal structure and classifications for anatase and rutile phases of $\mathrm{TiO}_{2}$ are shown in Figure 3. Although there is a third stable phase of $\mathrm{TiO}_{2}$, brookite, its stability and photoactivity are not sufficient for practical applications of photocatalysis. It has been recognized since 1964 that in the pure forms, anatase has the highest photocatalytic activity [8]. There are several proposed reasons for this. The most widely accepted is that the charge carrier lifetime in anatase is significantly greater than it is in rutile. This is due to the fact that rutile has a direct band-gap, whereas anatase has an indirect band-gap. 
This mismatch in k-space of the valence band maximum and the conduction band minimum slows the process of photo-excited electrons recombining with valence band holes [43].

However, pure anatase does not have the highest photoactivity of all forms of $\mathrm{TiO}_{2}$, it has been found that its activity is actually enhanced if the phase is a mixed anatase-rutile. The primary reason for the enhancement is believed to be a reduction in the electron-hole recombination rate due to hole trapping at the rutile-anatase interface [43]. Additionally, it has been shown that the effect is optimal at an anatase to rutile ratio of approximately 3:1, which can be obtained through controlled heat treatment of anatase crystals [44-45].

This effect is widely utilized in literature through the use of a commercially available form of $\mathrm{TiO}_{2}$ known as Degussa P25 - a nanoparticulate powder of pure $\mathrm{TiO}_{2}$, produced cheaply and in large quantities as a paint pigment via flame pyrolysis. Because of its high photoactivity and low cost, P25 is used both as a standard for reference and as a raw material for fabrication.

\subsection{2 $\mathrm{SiO}_{2}$ as Catalyst Support}

However, the choice of what material to coat the $\mathrm{TiO}_{2}$ onto is also extremely important for the effectiveness of the system. The role of the catalyst support material is to provide the 3D structure for the photocatalyst. Ideally, this is done in a way that provides high surface area, enhanced reaction kinetics, and optimal photonic efficiency. Since the support material does not directly take part in the catalysis reaction, it should be as transparent as possible to the wavelengths of light given off by the LED emitters. This has the effect of minimizing the parasitic absorption by the support, ensuring that the 
photocatalyst support does not create optical shadows within the reaction chamber. For example, for the LEDs with an emission peak at $365 \mathrm{~nm}$ (as used for this research), a support material that is highly transmissive of the wavelengths from 350 to $380 \mathrm{~nm}$ should be chosen.

There are several other material properties that must be considered, which include: 1) high chemical stability to prevent self-degradation, 2) low electrical conductivity to prevent energy loss during photocatalysis, 3) high photostability to prevent breakdown by UV illumination, 4) high temperature stability to allow for photocatalyst heat treatment during fabrication and cleaning, 5) high purity to prevent atomic migration into the $\mathrm{TiO}_{2}$ film during calcination, 6) high strength and durability to enable use of high water flow rate and water pressure, 7) the ability to be scaled for mass fabrication (low cost, nontoxic, abundant).

The most successful material used as a catalyst support for $\mathrm{TiO}_{2}$ is silicon dioxide $\left(\mathrm{SiO}_{2}\right)$ $[32,35,38,46-48]$. The primary reason for this combination is the extremely high UV transparency $\mathrm{SiO}_{2}$, which typically exhibits $\sim 93 \%$ transmission of UV light through a 1 cm plate, for wavelengths in the $350-380 \mathrm{~nm}$ range. Yet, there is also a material compatibility between $\mathrm{SiO}_{2}$ and $\mathrm{TiO}_{2}$ that is quite remarkable, as is discussed thoroughly in a review article by Gao and Wachs that analyzes a broad range of physical and chemical characteristics observed in various titania-silica composite materials [49].

The result of their analysis is that, unlike other catalyst support materials, the incorporation of $\mathrm{SiO}_{2}$ into the photocatalyst material has a beneficial effect on the photoactivity of $\mathrm{TiO}_{2}$. This is attributed to increased hydrophilicity at the catalytic surface 
and electron-hole pair separation at the $\mathrm{SiO}_{2}-\mathrm{TiO}_{2}$ interface $[45,49]$. However, the beneficial effect is only found for pure $\mathrm{SiO}_{2}$ substrates. On the other hand, it has been found that $\mathrm{TiO}_{2}$ films prepared on non-pure forms of silica, such as borosilicate or soda lime glass, exhibit highly reduced photoactivity due to atomic diffusion of impurities (e.g. $\mathrm{Ca}, \mathrm{B}, \mathrm{Al}$, etc) from the substrate into the $\mathrm{TiO}_{2}$ during the calcination process [50]. In addition, the UV transparency of these materials is reduced due to these impurities. Therefore, it is highly desirable to use one of the pure forms of $\mathrm{SiO}_{2}$ glass, such as quartz or fused silica when optimal energy efficiency is of concern. 


\section{Chapter 2: Hypothesis}

\subsection{Conceptual Basis}

There are several conclusions from Chapter 2 that are worth reiterating to establish context for the upcoming theoretical work: a) the determined experimental objective is to create a fixed catalyst material that is durable and capable of high photonic efficiency, b) the morphology chosen is that of a rigid 3D material occupying the volume a photoreactor chamber, c) a $\mathrm{TiO}_{2}-\mathrm{SiO}_{2}$ hybrid material was chosen based on high compatibility and ability to satisfy all the various functionality requirements, d) a fabrication method was chosen based on scalable fabrication principles that consists of dip coating fused silica rods with a Ti alkoxide sol-gel then assembling the $3 \mathrm{D}$ material into a cartridge.

Since the greatest problem with fixed catalyst systems has been low photonic efficiency, it is necessary to analyze the root cause of this issue. Consider a $\mathrm{TiO}_{2}$ nanoparticle, of 20 $\mathrm{nm}$ diameter, that is suspended in water. When a photon is absorbed by the particle and an electron-hole pair is generated, the carriers cannot be farther than $10 \mathrm{~nm}$ from the water interface. This situation possesses the ideal photon conversion efficiency, which is reflected in the relatively high photonic efficiency of slurry reactors.

However, in fixed catalyst systems the photocatalyst structure is much larger because it requires high strength and durability to withstand high flow rates and pressures. This means that carriers are, on average, generated further away from the catalyst-water interface, which decreases the probability that they will be able to reach the surface 
before recombination occurs. Additionally, any photons absorbed by the catalyst support will not generate carriers at all, and so this type of absorption is parasitic. Thus, for a 3D photocatalyst to approach the ideal photonic efficiency of a nanoparticle suspension, it is necessary to design the material in a way that minimizes losses from both recombination and parasitic absorption. Therefore, the following chapter presents a novel theoretical approach created to give a fundamental basis for choosing the geometrical and optical parameters of the final 3D material.

This model is a probabilistic approach used to establish a first order optimization for the efficiency of a 3D photocatalyst system by analyzing the effect of the film thickness on photonic efficiency for a given geometrical design. An important assumption of the model is that the optimization of the photonic efficiency for the system will also effectively optimize the degradation of the associated photocatalytic oxidation process. This is justified through a logical argument that the catalytic degradation rate is directly proportional to the number of available holes at the catalytic surface (i.e. the photocatalyst-water interface).

Thus, a major assertion of this theory is that the maximum degradation rate for a given geometrical structure will occur when the photonic efficiency, $\xi$, is maximized. Here, $\xi$ is defined as the ratio of the rate of oxidation reaction occurrence per area of catalytic surface, $S$, to photon flux on the catalytic surface, $\Phi$. The catalytic surface is defined as the 2-D surface at the interface of the 3-D photocatalyst material and the contaminated water. As we will see, $S$ changes when the thickness of the photocatalyst film changes, 
and so is a function thickness. Therefore, $\xi$ is also a function of thickness. This aspect will ultimately be utilized to formulate a hypothesis on efficiency optimization.

$$
\xi(t) \equiv \frac{S(t)}{\Phi}
$$

The work below presents a simple situation where the incident light is perpendicular to the catalytic surface, and the photocatalytic material is a uniform thin-film coated onto a flat substrate. For thin films on the order of $100 \mathrm{~nm}$, and silica rods of $1 \mathrm{~mm}$ diameter, this approximation is reasonable given that the ratio of the radius of curvature to film thickness is $>10,000: 1$. Though this situation is simplified from a real system, it is very helpful for understanding the important principles that lead to optimization of systems photonic efficiency, and is sufficient for providing a first order approximation for the actual geometry of the material that will be presented Chapter 3 .

A useful way of approaching this model is to think of an individual incoming photon, $\gamma$, as a packet of energy, and that this model calculates the probability that this energy will pass through the catalytic surface, be absorbed within the photocatalyst material, and return to the surface of the material in the form of an electron-hole $(e-h)$ pair. This process requires several steps. The simplest mechanism would be the process of absorption, $e-h$ pair formation, and diffusion of charge carriers to the surface. However, this process might also occur through more complicated pathways, which could contain various combinations of processes, (e.g. scattering, luminescence, recombination, etc.).

The elegance of the approach is to encapsulate all of these possible processes into a simple probability theory that only requires consideration of the spatial translation of the 
energy, and doesn't require that the mechanism of the translation is known. This is accomplished by statistically partitioning the process into 2 independent events: 1) the translation of the energy through the material in the form of a photon, and 2) the transfer of the energy from the absorption point within the material to the catalytic surface in the form of $e-h$ pair. However, the simplicity that is introduced by this approach also limits the applicability of the model, and therefore the quantitative results are strictly only valid for planar systems - though it will be argued that these results do provide a good approximation for the complicated geometry of the real system design. At the very least, the results qualitatively useful for gaining a deeper understanding of the processes that affect the system efficiency.

\subsection{Theoretical Concepts}

In this section, a conceptual description of the model is given. After these ideas are established, mathematical definitions will be established from empirical data. To start, consider a point, $A$, somewhere within the photocatalyst shown in Figure 4. The material is a flat catalyst support with a thin photocatalytic coating. This point, $A$, is located at a depth, $x$, within the photocatalyst film as measured from the catalytic surface, which is the 2D surface that comprises the water-photocatalyst interface If it is assumed that this catalytic surface is uniform in terms of morphology and material composition and that the intensity of incoming photons is uniform on the catalytic surface, then because of the system symmetry, this point is identical to all other points in the photocatalyst located at

a depth $x$. These points form a plane that is parallel to the catalytic surface and separated from it by a distance $x$ - this plane will be referred to as the absorption plane. 
Assuming that the material is uniform, the rate of photon absorption at the absorption plane is proportional to the intensity of photons incident on the plane. Therefore, as $x$ increases, the intensity of photons on the absorption plane decreases, and so the rate of e$\mathrm{h}$ pair generation decreases with increasing distance from the catalytic surface. Thus, for an incoming UV photon, the probability of being absorbed is a monotonically decreasing function of $x$. This mathematical form of this function will later be determined through empirical data, but for now will be named the generating function, $g(x)$.
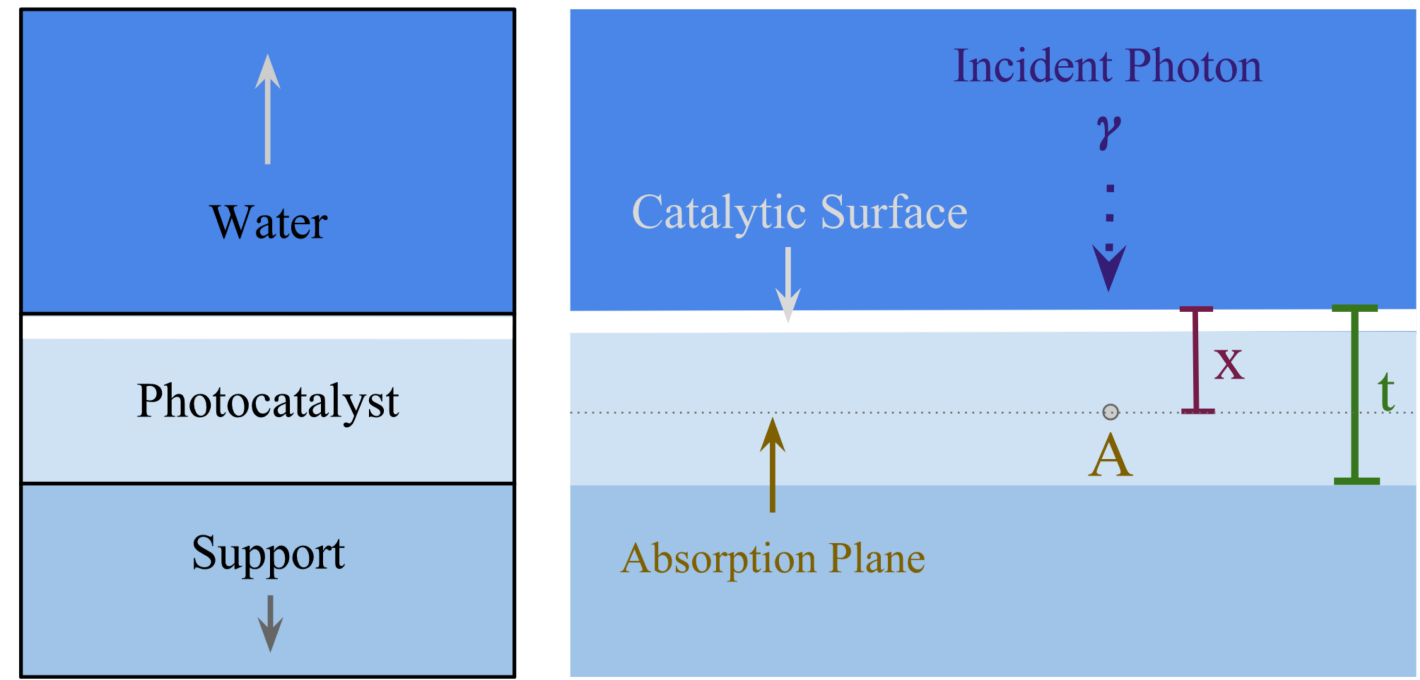

Figure 4: diagram clarifying the basic geometry of the photocatalyst interface and spatial variable definitions considered for the basis for the theoretical model.

Now, let's assume that the absorption of $\gamma$ results in the promotion of electron from a valence state to a conduction state, and the generated carriers are now free to diffuse from the generation point, $A$. The probability of this $e-h$ pair traveling to the catalytic surface is primarily dependent upon the minority carrier $\left(h^{+}\right)$diffusion length, and becomes decreasingly probable as the generation distance, $x$, increases. So the probability of a given $e-h$ pair reaching the surface is again a monotonically decreasing function of $x$. This will eventually be defined based on statistical simulation, but for now will just be 
named the surfacing function, $s(x)$. Since these two processes, $s(x)$ and $g(x)$, are statistically independent, the probability of an incoming photon generating an $e-h$ pair at a depth $x$ AND the resulting $e$ - $h$ pair diffusing to the catalytic surface is determined by the product of the generating function and the surfacing function. This product can be understood as the process probability density, defined below as $p(x)$.

$$
p(x)=g(x) s(x)
$$

For a particular photon, $\gamma$, the probability, $P$, that its energy will be successfully converted into an available $e-h$ pair at the catalytic surface can be found through the integration of $p(x)$ over all $x$; thus the total probability of a successful energy transformation is a function of the photocatalyst thickness, $t$, and is defined by the integration of $p(x)$ from 0 to $t$.

$$
P(t)=\int_{0}^{t} p(x) d x
$$

This allows for a probabilistic interpretation of the photonic efficiency for the system. If the rate of incoming photons, rate of photon absorbance, and rate oxidation reaction occurrence are all in a steady state - which can be assumed to be true if the rate photodegradation is constant - then the rate of oxidation occurrence, $S$, can be found by multiplying $P(t)$ by the photon flux, $\Phi$. Now, substituting $S(t)$ into Equation (18) provides a useful expression for $\xi$ in terms of $g(x)$ and $s(x)$.

$$
S(t)=P(t) \Phi
$$




$$
\therefore \xi(t)=\int_{0}^{t} g(x) s(x) d x
$$

Therefore, the photonic efficiency for the system is also a function of the film thickness, and can thus be optimized if $\xi(t)$ has maxima in the practical range of film thickness. For a single photocatalyst film, $\xi(t)$ should asymptotically approach a maximum value, this is because increasing the film thickness only has the effect of extending the depth being integrated over, without any detriment to $\xi$. Indeed, this has been shown to be true empirically [51-52]. However, in the more complex and interesting systems, such as with the multilayered materials that will be analyzed subsequently, more meaningful behavior will be observed. Before analyzing these results more thoroughly, it is necessary to define and justify $g(x)$ and $s(x)$ mathematically. 


\subsection{Defining the Probability Functions}

\begin{tabular}{c|l} 
Symbol & \\
\cline { 1 - 1 } $\boldsymbol{\xi}$ & Photonic efficiency \\
\hline$\Phi$ & Photon flux on $\mathrm{TiO}_{2}$ film (units of $\left.\mathrm{s}^{-1} \mathrm{~m}^{-2}\right)$ \\
\hline$S$ & Rate of oxidation reaction occurrence per area of catalytic surface (units of $\left.\mathrm{s}^{-1} \mathrm{~m}^{-2}\right)$ \\
\hline $\boldsymbol{g}(x)$ & Mathematical Variable: penetration distance by incoming UV photon \\
\hline$s(x)$ & Probability of individual photon absorption at depth of $\boldsymbol{x}$ \\
\hline$p(x)$ & Probability of an electron-hole pair, generated at depth $\boldsymbol{x}$, reaching catalytic surface \\
\hline$P(t)$ & Probability of converting UV photon for $\mathrm{TiO}_{2}$ film of thickness $\boldsymbol{t}$ \\
\hline$t$ & Parameter to be Optimized: thickness of $\mathrm{TiO}_{2}$ film \\
\hline$I(x)$ & Intensity of $\mathrm{UV}$ photons as depth of $\boldsymbol{x}$ \\
\hline$\alpha$ & Empirical constant related to UV attenuation coefficient \\
\hline$a$ & Normalization constant for $g(x)$ function \\
\hline$\beta$ & Empirical constant related to diffusion coefficient of $e-h$ pairs in ceramic $\mathrm{TiO}_{2}$ film \\
\hline$b$ & Scaling coefficient for $s(x)$ function \\
\hline
\end{tabular}

Table 2: Symbol definitions used in the theoretical model.

\subsubsection{The generating function: $g(x)$}

To reiterate, $g(x)$ represents the probability that an incoming photon will be absorbed at a depth of $x$, within the material. In order to quantify the generation function, $g(x)$, it is necessary to consider the intensity, $I$, of UV photons within the photocatalyst material. For the following argument consider a uniform illumination of the catalytic surface with a coherent beam of UV photons. Because of the system's symmetry, all points at depth of $x$ will have the same light intensity, and so the intensity function is independent of the $y$ and $z$ coordinates. Also, because the illumination source for the system is LED based 
with a narrow emission band, $I$ does not require the usual frequency dependence, as values specifically for $\lambda=365 \mathrm{~nm}$ can be used.

Thus, $I$ is a function only of depth and can be written as $I(x)$. It is apparent that as $\mathrm{x}$ increases, $I(x)$ must decrease monotonically since photons can only be removed from the signal, e.g. through absorption, reflection, or scattering. For the case at hand, it is not relevant which mechanisms are responsible for the intensity depreciation, it is only necessary to determine the mathematical form of the depreciation. This is because the probability of a photon being absorbed at depth $x$ is proportional to the intensity of photons at that depth. This assertion assumes that the photocatalyst material is uniform and that the absorption cross section is homogeneous throughout the material. However, since this intensity function represents the probability of a single photon being absorbed at a depth of $x$ within the material, a normalization constant, $a$, must also be included. Thus we arrive at a generic generation function.

$$
g(x)=a I(x)
$$

Next, it is necessary to determine the mathematical form of $I(x)$, in order to know the generation function's dependence upon $x$. For a uniform material, such as the one being considered, it is appropriate to utilize the Beer-Lambert relation, which shows that the attenuation of light within a material is represented by an exponential decay function. This is valid for uniform materials that can be shown to display a linear relationship between absorbance and film thickness.

$$
I(x)=I_{0} e^{-\alpha x}
$$


This has been verified empirically for thin films of $\mathrm{TiO}_{2}$. This fact allows for the quantification of the light intensity within the material as a function of $x$ by determining the decay constant for the material. In this work, this constant is denoted by the greek letter alpha, $\alpha$, and is one of the two empirical parameters needed for the optimization of the $\xi(t)$. The value of was determined from data shown in Figure 5, originally presented by Zhang et al., who used the same Ti sol-gel precursors used for this work [53].

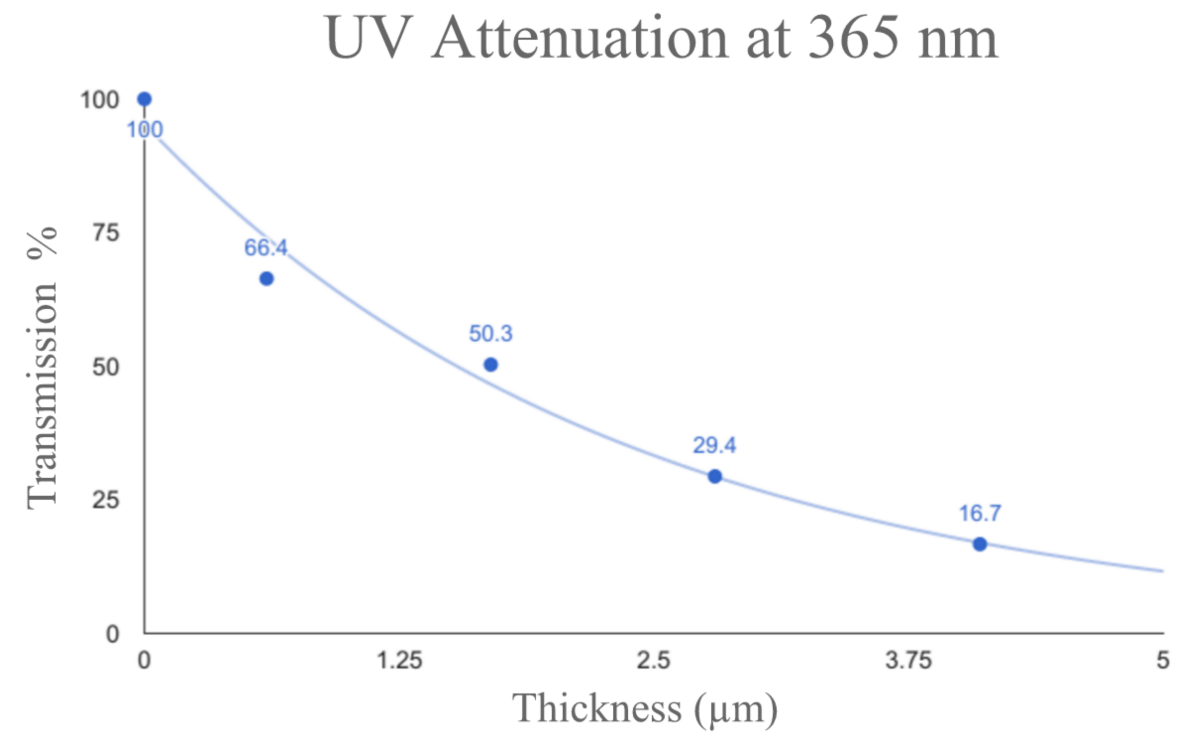

Figure 5: Empirical measurements of UV attenuation $(\lambda=365 \mathrm{~nm})$ by sol-gel derived $\mathrm{TiO}_{2}$ films of various thickness, allowing for determination of $\alpha$ in $g(x)$. Data reproduced from Zhang et al. [53].

The normalization constant can now be determined by considering the fact that for a film of infinite thickness the probability of a photon being absorbed in the film must be unity. Therefore by integrating the intensity function over $x$ from 0 to infinity, setting the result equal to one, then solving for $a$.

$$
\begin{aligned}
& \int_{0}^{\infty} a I_{0} e^{-\alpha x} d x=1 \\
& \therefore a=\frac{\alpha}{I_{0}}
\end{aligned}
$$


Finally, after inserting the normalization constant and Equation (24) back into the Equation (23), the generation function takes the following form.

$$
g(x)=\alpha e^{-\alpha x}
$$

\subsubsection{The surfacing function: $s(x)$}

As described above, the surfacing function represents the probability that the energy absorbed from the incoming photon will be successfully transported from the absorption site, $A$, to the catalytic surface. As with the collection probability for solar cells, this probability is determined by the minority carrier diffusion length, which is dependent upon the $h^{+}$conductivity for the material. Thus, for a uniform material, it must be a monotonically decreasing function of $x$. This can be understood by considering a minority carrier that is generated at depth $\mathrm{x}$. The probability that the carrier will surface must decrease as the distance of $A$ from the surface increases. A stochastic random-walk simulation was done to determine that the surfacing probability for a $\mathrm{TiO}_{2}$ film is also an exponential decay function.

$$
s(x)=b e^{-\beta x}
$$

The coefficient, $b$, can be determined logically by noticing that for carrier generated at a depth of 0 has a $100 \%$ probability of surfacing because it is already at the catalytic surface: therefore $b$ must be equal to unity.

$$
\begin{aligned}
& b=1 \\
& \therefore s(x)=e^{-\beta x}
\end{aligned}
$$


Unlike $g(x)$, the $s(x)$ function does not need to be normalized because the probability of surfacing at one point within the material is independent of the surfacing probability of the other points. Therefore, $s(x)$ can be simply represented by the decay constant $\beta$, which is the second material parameter necessary for system optimization. In the argument presented below, the beta constant will be determined semi-empirically from photocatalytic degradation experiments conducted on films of varying thickness. But to do so, we must first consider the details of the process probability function.

\subsubsection{The process probability density: $p(x)$}

The process probability density represents the probability that a given photon will be absorbed at depth $x$ AND that this energy will return to the catalytic surface by means of charge carrier diffusion. Because these two processes are independent, the total probability for the process occurring is the product of $g(x)$ and $s(x)$. Therefore, from Equation (19), $p(x)$ can be determined.

$$
p(x)=\alpha I_{0} e^{-\alpha x} e^{-\beta x}
$$

\subsubsection{The Total Probability: P(t)}

Now, in order to calculate the total probability, $P$, that a given photon will be absorbed anywhere within the material and that the carriers will diffuse to the surface, requires integration of $p(x)$ in order to sum over the entire range of $x$ that is of interest. For a film of thickness, $t$, this range is from 0 to $t$. Notice that $P$ is necessarily a function of $t$, and that $P(t)$ increases monotonically with increasing film thickness, as predicted. 


$$
\begin{aligned}
& P(t)=\alpha \int_{0}^{t} e^{-\alpha x-\beta x} d x \\
& P(t)=\frac{\alpha\left(1-e^{-t(\alpha+\beta)}\right)}{\alpha+\beta}
\end{aligned}
$$

\subsubsection{The photonic efficiency: $\xi(t)$}

As described above, calculation of $P(t)$ relates directly to photonic efficiency for the system. This can now be seen explicitly by substituting Equation (21) into Equation (18).

$$
\begin{aligned}
& \xi(t)=\frac{S(t)}{\Phi}=P(t) \\
& \therefore \xi(t)=\frac{\alpha\left(1-e^{-t(\alpha+\beta)}\right)}{\alpha+\beta}
\end{aligned}
$$

Recall that the primary assertion of this model is that this quantity should be directly proportional to the photoactivity of the film. The theory can now be tested against experimental data. Kozawa et al. measured the degradation rate of $\mathrm{TiO}_{2}$ films as a function of thickness; this data has been re-plotted from their values in Figure 6 [53]. From this plot, it can be seen the trend of the data is in agreement with that predicted by Equation (35): that is, asymptotically approaching the maximum degradation rate as thickness increases. To verify agreement of Equation (35) with this empirical data, a nonlinear regression was applied. The result is shown along with the data in Figure 6. The Rsquared of 0.98 provides support for the assumptions used for the derivation of Equation (34). From this fit, it was then possible to extract the $\beta$ parameter from this the data since an approximation of $\alpha$ was previously determined from Figure 5. 


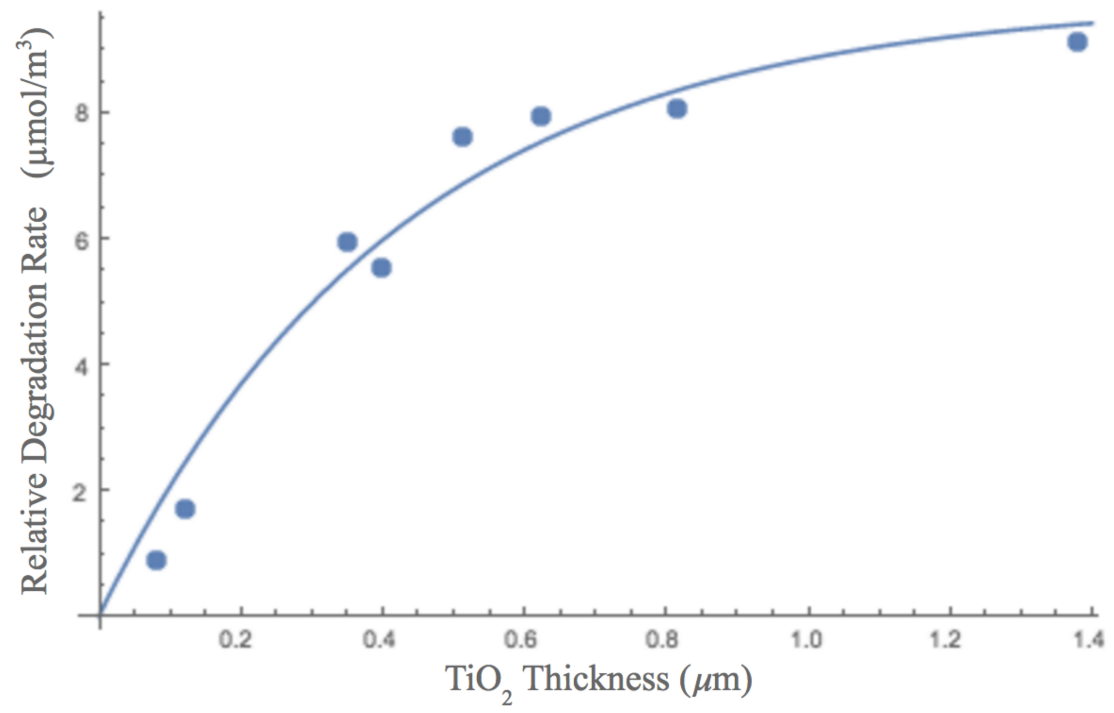

Figure 6: Plot of photodegradation rate vs film thickness for $\mathrm{TiO}_{2}$ film derived by $\mathrm{TiO}_{2}$ nanoparticle immobilization (replotted from Kozawa et. al [53]) and a nonlinear regression line obtained by fitting Equation (35) to the data.

\subsection{Support-Side Illumination}

Now the basis of the theoretical model has been established, it can be applied to more interesting situations that provide more significant information. The first step in understanding how this can be done is to reconsider the situation presented in Figure 4. Recall that in this situation, UV illumination was incident on the photocatalyst surface that is exposed to the water. Now consider how the model will change if the illumination is incident on the photocatalyst surface that is in contact with the support structure, as shown in Figure 7.

There are two important things to understand about this: 1$)$ the $s(x)$ function will not change with respect to the original situation because the carriers still need to diffuse to the same catalytic surface; 2) the $g(x)$ function will change because the light intensity is 
now greatest at the photocatalyst-support interface, rather than at the photocatalyst-water interface as before. This
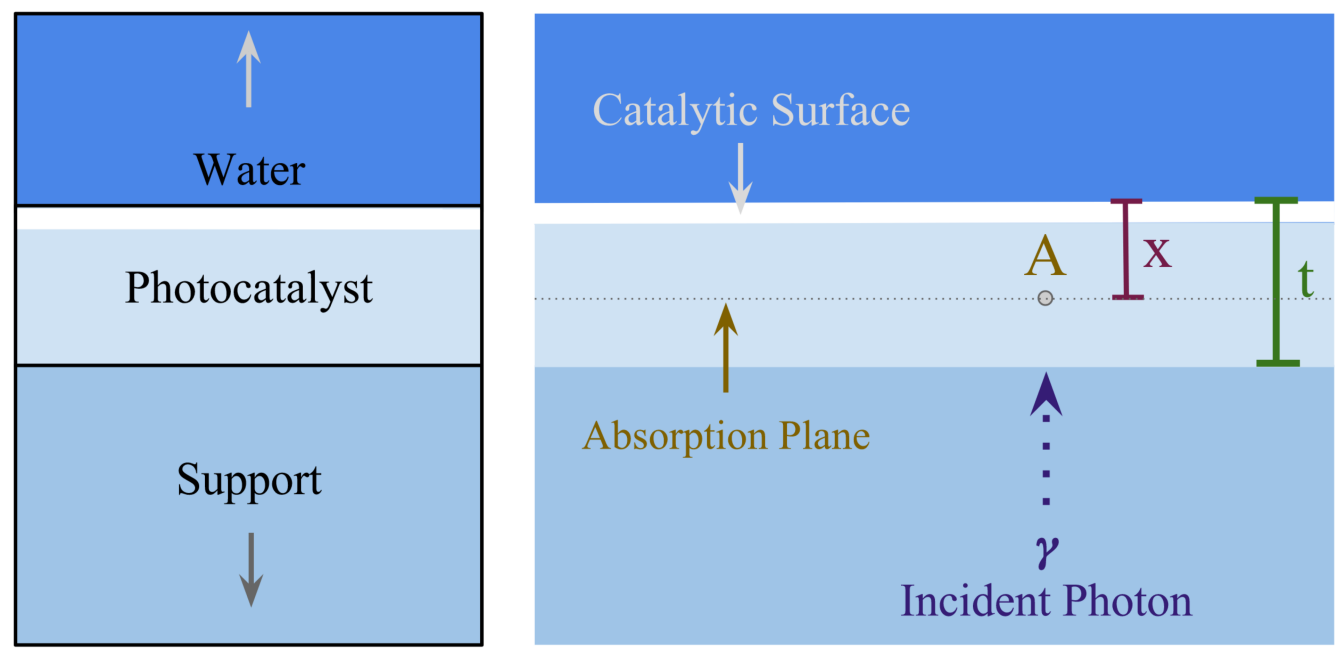

Figure 7: a modification of the diagram shown in Figure 4, where the incident photons are now supplied from the catalyst support side of the photocatalyst.

difference alters Equation (24) and thus Equation (27) so that the relative intensity is unity when $x=t$ and exponentially decays as $x$ approaches zero.

$$
g(x)=\alpha e^{\alpha(x-t)}
$$

Now, following the same steps used to derive Equation (35), results in a new expression for the photonic efficiency under support-side illumination (Equation (37)).

$$
\xi(t)=\frac{\alpha\left(e^{-\beta t}-e^{-\alpha t}\right)}{\beta+\alpha}
$$

The effect of these changes is significant because $\xi(t)$ now has a global maximum rather than asymptotically approaching a maximum value. That is, there exists a maximum photonic efficiency for a specific value of photocatalyst thickness. This can be seen by 
plotting $\xi(t)$ using the values of $\alpha$ and $\beta$ extracted from Figure 5 and Figure $6(\alpha=1.54$ $\mu \mathrm{m}^{-1}$ and $\left.\beta=0.85 \mu \mathrm{m}^{-1}\right)$. The result is shown in Figure 8.

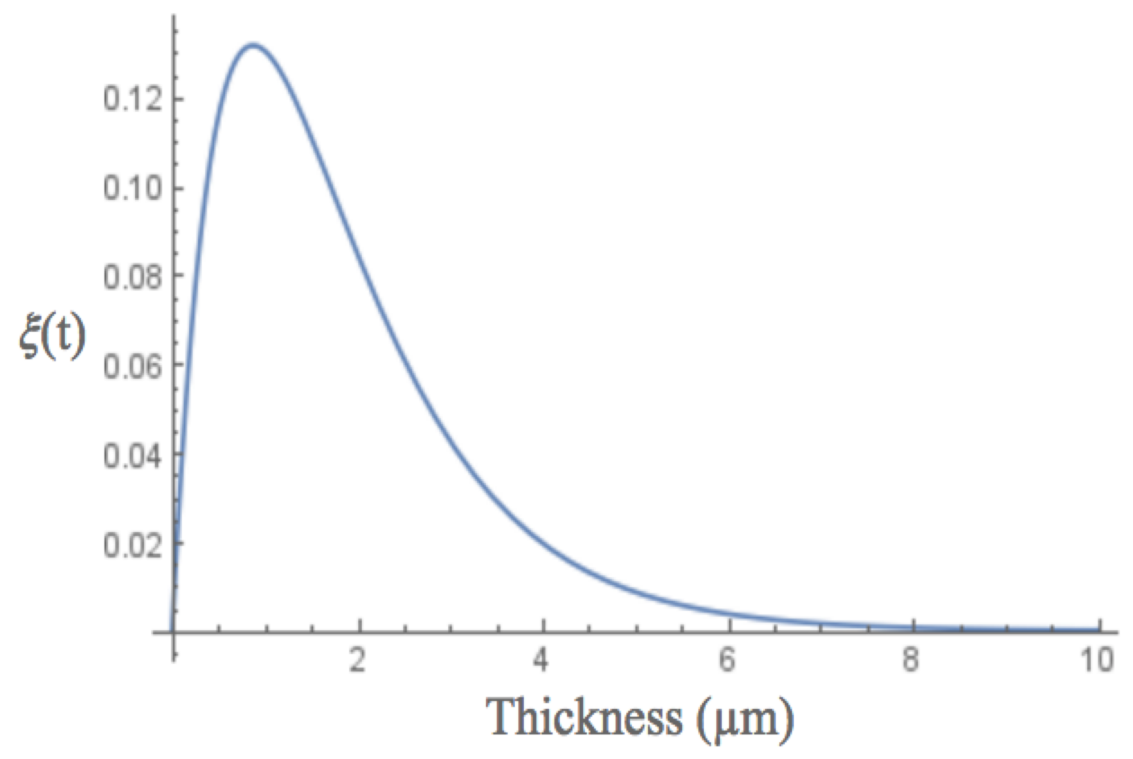

Figure 8: Hypothetical plot of the photonic efficiency as a function of film thickness for support-side illumination setup presented in Figure 7.

Evidence of this behavior was found in a study conducted by Tada, et. al, who experimented with a very similar scenario [54]. For their work, $\mathrm{TiO}_{2}$ films with various thicknesses were prepared on quartz slides. The hydrophilicity of the films was tested by applying a water droplet to a horizontal sample and measuring the water contact angle while illuminated with UV from below. Since the water contact angle is correlated with photoactivity for $\mathrm{TiO}_{2}$ films [55], their results are highly relevant. Indeed, the data they presented is in high agreement with the general shape of the plot in Figure 8.

However, the maximum hydrophobicity of their plot does not occur at around $1 \mu \mathrm{m}$, but at a much thinner thickness of approximately $100 \mathrm{~nm}$. The reason for this is due to the difference in type of $\mathrm{TiO}_{2}$ films used: in the research used to find the $\alpha$ and $\beta$ constants 
the films were derived by $\mathrm{TiO}_{2}$ particle immobilization and in the work by Tada, the films were derived from a Ti sol-gel. Although this highlights the fact that these results are not quantifiable, the extremely close match of the empirical data to the overall hypothetical behavior suggests that the reasoning presented is sound.

Consider the logical differences between the case of water-side illumination and support side illumination. When the light is incident on water-photocatalyst interface it is appropriate to have a thick photocatalyst film since $\xi(t)$ asymptotically approaches a maximum value. Additionally, the material used for the catalyst support is nearly irrelevant because the optical properties of the support do not have any effect on $\xi(t)$ and because for thick films, the material interaction of the substrate and the photocatalyst have negligible effect. On the other hand, for support-side illumination there does exist an optimal film thickness. And in this case, the material properties of the support material have a great impact on $\xi(t)$. This is due to the attenuation of UV as it passes through the support material, and the high impact of substrate-photocatalyst material interaction for thin films.

Thus, for a 3D photocatalyst material, the concepts outlined above must be taken into account. The next step in implementing this theory is to apply it to systems of multiple layers in order to observe how the material properties of the water and substrate affect the optimization results. 


\subsection{Multi-Layer Systems}

\subsubsection{System Descriptions}

The first multi-layer system considered is a 3-layer material as depicted in Figure 9, which will then be extended a 13-layer system that is relevant to $3 \mathrm{D}$ photocatalyst material that will be presented in Chapter 3. The 3-layer material can be imagined as a glass slide coated on both sides by a photocatalyst material. Though not considered yet, it can be assumed that there is water to the left and right of this material.

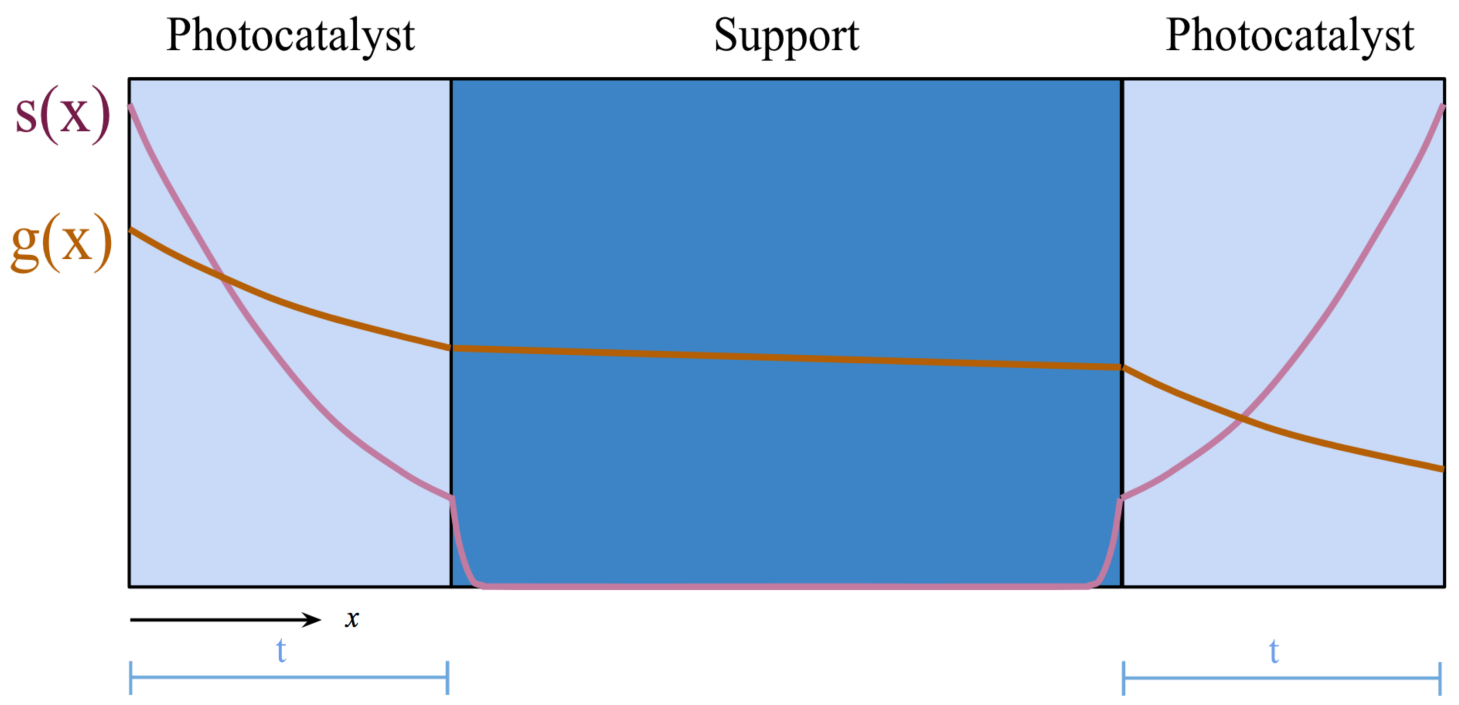

Figure 9: Schematic diagram showing the geometry of the 3-layer system, along with qualitative representations of the $g(x)$ and $s(x)$ function within the material.

As before, this situation assumes that the support material is flat and uniform so that the concept of the absorption plane that was presented in Section 2.2 is still valid. Therefore, the values of generating function and the surfacing function are again only dependent on the distance from the catalytic surface, $x$. However, in this case, there are two catalytic surfaces, so $x$ is chosen to be measured from the left-hand side of the diagram where the 
illumination is incident on the material. To clarify, qualitative plots of $g(x)$ and $s(x)$ functions are also shown.

From these representations it is clear the probability a photon being generated, $g(x)$, exponentially decays as the depth within the material $(x)$ increases. Notice, however, that at the photocatalyst-support interface (where $x=t$ ), both $g(x)$ and $s(x)$ change dramatically due to differences in UV attenuation and carrier mobility between the photocatalyst and support material. This is reflected in the fact that the constants $\alpha$ and $\beta$ take on different values in this region. Since the support material is chosen to be highly UV transparent, $g(x)$ only depreciates slightly in this region. On the other hand, $s(x)$ drops immediately to zero, because the support material is an insulator and so the electrical conductivity in this region is negligible.

Further, this means that the contribution of the support material to photonic efficiency calculation is zero, since process probability $p(x)$ is a product of $s(x)$ and $g(x)$, as would be expected. However, it is worth pointing out that when in the region of the second photocatalyst layer, $s(x)$ begins to increase because $x$ is now approaching the second catalytic surface at the rightmost boundary of the material. This creates a situation that is identical in nature to that described in Section 2.4. Thus, calculation of $\xi(t)$ can be carried out in exactly the same manner as before, with the exception that it is calculated from the sum of each region's individual contribution (non-photocatalyst regions are ignored since $p(x)=0$ within the support material).

Developing the model further to consider more layers is simply an extension of these concepts. For example, if the 3-layer material is placed in a volume of water along with 2 
replicas, spaced evenly apart, then you will arrive at the 13-layer system shown in Figure 10. The same principles apply to this situation except that $\alpha$ and $\beta$ must take on appropriate values in the water regions, just as was the case for the support material in the 3-layer example. As before, the effect of this is that although the intensity of UV depreciates in those regions, this attenuation is parasitic as absorption of photons in these regions does not contribute to $\xi(t)$.

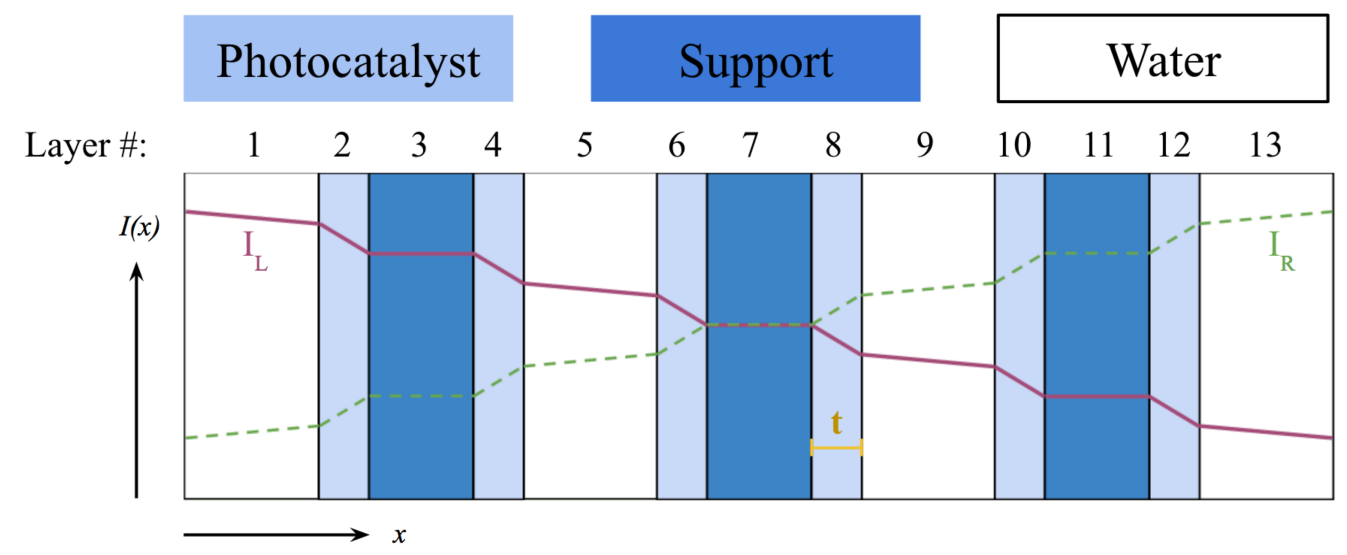

Figure 10: Diagram showing the geometry of the 13-layer system, including a qualitative representation how the intensity of light is attenuated as it passes through each layer. A situation in which illumination is incident from both the left side $\left(I_{L}\right)$ and the right side $\left(I_{R}\right)$ is considered.

Additionally, Figure 10 also shows that illumination can be provided from both sides of the system: $I_{L}$ represented UV illumination incident on the left-hand side and $I_{R}$ incident on the right-hand side. The effect of this consideration is that total intensity $I(x)$ is actually a superposition of these two functions. However, due to the symmetry of the situation, it was found that this had no overall effect on the photonic efficiency of the material. Although the rate of oxidation reaction occurrences, $S(t)$, was doubled by this addition, the photon flux, $\Phi$, was also twice as high. Thus, including this attribute had no overall effect on $\xi(t)$. 


\subsubsection{Calculations of the Photonic Efficiency}

The computations of $\xi(t)$ for both the 3-layer and 13-layer systems are shown in Figure 11. Analyzing these results for the 3-layer system shows that there exists a maximum in photonic efficiency as expected. Notice that as the thickness of the film increases, the total photonic efficiency approaches the value of the first layer. This is a logical conclusion, as it shows that if the film is thick ( $>8 \mu \mathrm{m})$, then no light is transmitted through the first layer and so the third layer is not able to contribute to the reaction.

a)

$\xi(\mathrm{t})$ for 3 Layer System

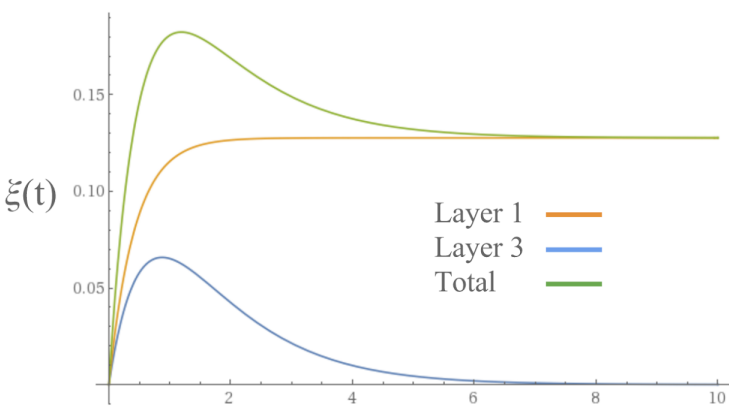

Thickness $(\mu \mathrm{m})$ b)

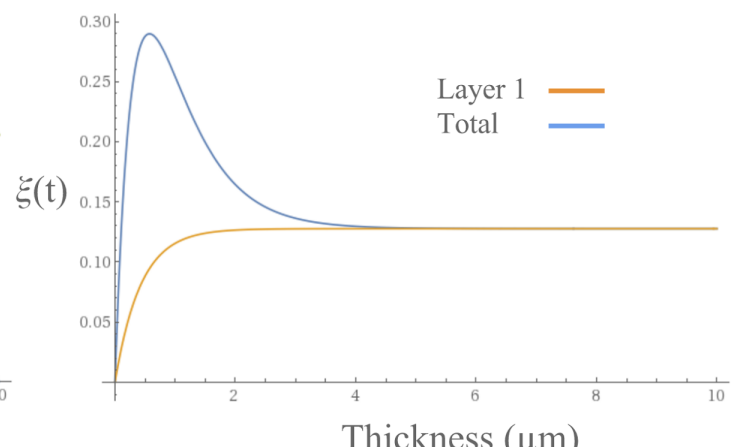

Figure 11: Calculation of the photonic efficiency as a function of thickness for a) the 3layer system shown in Figure 9, and b) the 13-layer system shown in Figure 10.

As for the 13-layer system results, it is important to notice that the optimal thickness value is less than that for the 3-layer system, but the maximum of value $\xi(t)$ is significantly greater: $\xi_{\max }(t)=0.183$ at $t=1.24 \mu \mathrm{m}$ for the 3-layer and $\xi_{\max }(t)=0.290$ at $t$ $=0.63 \mu \mathrm{m}$ for the 13-layer). This represents a $58 \%$ increase in efficiency for the 13 -layer system. Logically, this trend would continue if systems with even more layers were analyzed. That is, the efficiency of the system will always increase for systems with more layers of decreased photocatalyst thickness. This is not a surprising conclusion, however, as it was already discussed in Chapter 1 that photocatalyst particle suspensions provide 
the ideal efficiency, and continuing to increase the number of layers with decreased thickness will eventually lead to a planar approximation of this situation.

Thus, it can be concluded from this theoretical investigation that the creation of a $3 \mathrm{D}$ photocatalyst material can lead to increased photonic efficiency, but only if the optical and electrical properties of all materials are considered. Further, the efficiency will continue to increase as the material structures become smaller and smaller. This trend would likely change when the photocatalyst dimensions become too small to semiconducting behavior. However, this does not consider the strength and durability of the material, which must be able to withstand high water pressures and flow rates for industrial application. Therefore, the following chapter delves into the design principles that must be adhered to for the purpose of application, and then presents the actual design chosen for implementation. Considerations on the application of the theoretical model described in the current chapter, to the chosen design, are then presented at the end of Chapter 3. 


\section{Chapter 3: Photoreactor Design Principles}

Although many individual aspects photocatalytic purification process are fairly well understood in isolation, the creation of a system that accounts for all aspects simultaneously has not yet been achieved. The specific issues that have impeded application of this process include the following: 1) system throughput, 2) process efficiency, 3) catalyst durability, and 4) catalyst fouling. It is necessary that these aspects be considered together since the optical and rheological aspects of the device require careful consideration of the system's shape and geometry. It has typically been the case that attempting to optimize for one of these impediments leads to inhibition of one or more of the others. Hence, this a very interdisciplinary challenge. An effectual solution must include an understanding of how these disparate fields interplay to create an efficient energy transfer between several states: from electromagnetic energy as incident photons, to electrical energy within the semiconductor, then to chemical energy at the semiconductor-water interface and as ROS molecules in an aqueous solution.

\subsection{Novelty}

\begin{tabular}{|ll|}
\hline \multicolumn{1}{|c}{ Efficiency Aspects } & \multicolumn{1}{c}{ Scalability Aspects } \\
\hline Catalyst-surface-area to water-volume ratio & Simple and inexpensive fabrication process \\
High apparent quantum yield & Cheap, abundant, and non-toxic materials \\
Ability to modify and enhance catalyst surface & Easily replaceable \\
Geometrical alignment of catalyst and illumination & Regenerative catalyst surface \\
Minimization of photon losses & Modular design \\
\hline
\end{tabular}

Table 3: List of design aspects adhered to for the design of the 3D photocatalyst material, providing high potential for commercial or industrial application. 
A novel photocatalytic purification system consisting of a 3D photocatalyst material and a corresponding photoreactor, which considers both efficiency and scalability concerns, was created and is presented in this work. The particular aspects that have been considered are shown explicitly in Table 3.

The result is a photoreactor that utilizes an innovative hybridization of known materials and methods to produce a simple but effective photocatalytic purification system. This design addresses the common difficulties experienced by photocatalyst systems such as low quantum yield, mass transfer limitation, and catalyst fouling, while simultaneously addressing the optical, electrical, and chemical aspects of the degradation process. A rendering of the final reactor design is shown in Figure 12.

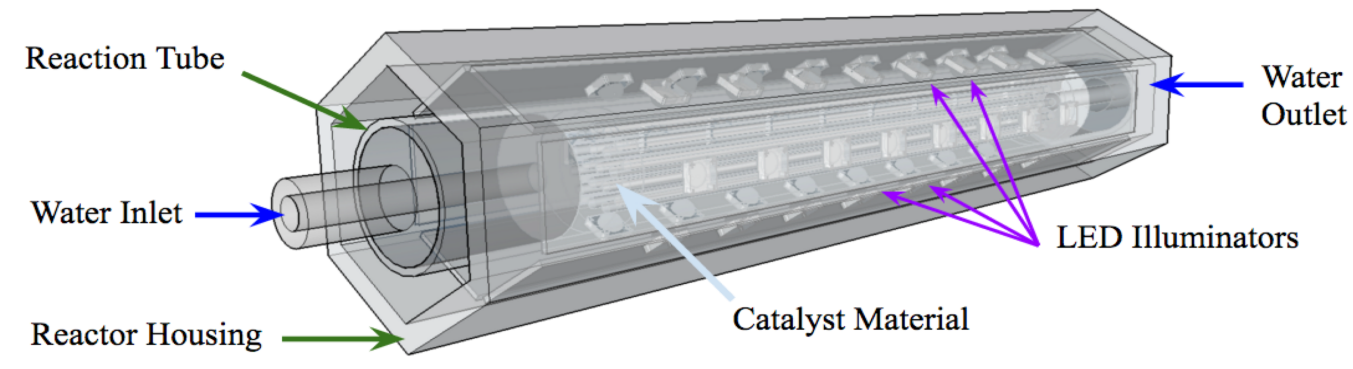

Figure 12: Overview of the photoreactor design showing the $3 D$ photocatalyst materials occupying the volume of the reaction chamber at the focus of the LED illumination system.

The design of the system presented is centered around the fine control of total photoefficiency through the development of a 3-dimensional photocatalyst. This design includes three particularly important novelties. 1) The first novel aspect of this system is the reactor's geometrical design; specifically, the geometry of the illumination system is complementary to the shape of a 3D photocatalyst material, providing a high ratio of 
catalyst surface area to water volume. 2) The second novelty is the implementation of a photocatalyst cartridge which can be easily removed from the chamber, providing the option for both chemical and heat treatment for reactivation processing or replacement. 3) The third novelty is the modular design of this reactor: a single reactor module can be easily numbered up to suit specific applications by connecting individual modules together in any combination of parallel or series connections. Together these novelties create an efficient yet practical photocatalyst system that addresses each of the 4 impediments identified in the introduction to this chapter.

\subsection{Description of Photoreactor System}

Based on a combination of a) the implementation requirements described in Chapter 1 and b) the results of the theoretical model presented in Chapter 2, a photoreactor system has been designed with the intention of creating a scalable and efficient degradation system. In Figure 13, the housing has been removed from the image to show the configuration of the illumination system and the 3D catalyst material more clearly. The illumination system is composed of 6 strips of ultraviolet LEDs $(365 \mathrm{~nm})$ arranged in a hexagonal fashion that focuses the emitted photons on the center of the reaction chamber. The reaction chamber itself is composed of a quartz tube that contains a photocatalyst cartridge - a 3D silica-titania composite consisting of 19 fused silica rods that have been coated with a thin-film of titania photocatalyst. 


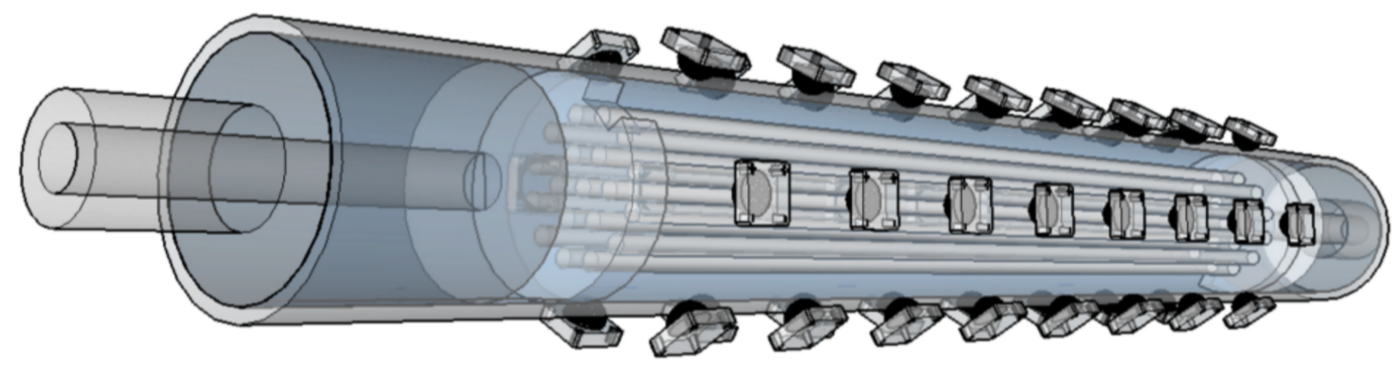

Figure 13: a) rendering of the photoreactor showing an overview of the primary design aspects. UV-LEDs surround a reaction tube, which contains the $3 D$ photocatalyst material.

The six UV-LED strips are arranged into a hexagonal tube that surrounds the reaction chamber, giving the illumination system geometry 6-fold rotational symmetry. This aligns with the rotational symmetry of the photocatalyst material that is inside the reaction tube. Therefore, with the led strips and the catalyst bundle properly aligned, the entire system shares a 6-fold rotational symmetry. This is described with more detail in Section 3.4.

Photons emitted by the illumination system are absorbed by the photocatalyst film. Because the photocatalyst is a semiconductor, this absorption results in the generation of electron-hole pairs within the material since the energy of the emitted photons $(3.4 \mathrm{eV})$ is greater than the band gap of the titania photocatalyst $(3.2 \mathrm{eV})$. Degradation of a pollutant can then occur through either of two primary mechanisms: 1) direct oxidation of the pollutant via interaction with the electron hole $\left(h^{+}\right)$at the photocatalyst surface, or 2) oxidation of the pollutant via interaction with hydroxyl radicals $(\mathrm{OH} \bullet)$ that are produced when water molecules themselves react with the photocatalyst [3, 19]. As pollutant molecules move through the chamber they are subjected to successive oxidation reactions of this type. This process is considered to be non-selective; therefore, if the residence 
time within the chamber is sufficient, all organic molecules will become mineralized (i.e., complete conversion of the pollutant molecules into $\mathrm{H}_{2} \mathrm{O}, \mathrm{CO}_{2}$, and simple mineral acids [14]).

\subsection{Photocatalyst Cartridge}

An important novelty of this design is the use of a photocatalyst cartridge, shown in Figure 14, which was designed to be both removable and regenerable. The catalystsupport is a rigid material in the shape of a rod bundle, which provides the shape, strength and durability of the cartridge. Because the photocatalyst material itself is in the form of a thin coating on the exterior of the support, it takes on the same 3-dimensional shape as the support material. Since the photocatalyst material is highly absorptive of the UV light, its shape and quantity are extremely important aspects of the cartridge design. The shape of the rod bundle minimizes water pressure on the film surface, which improves the lifetime of the photocatalyst film as this prevents film cracking and flaking.

a)

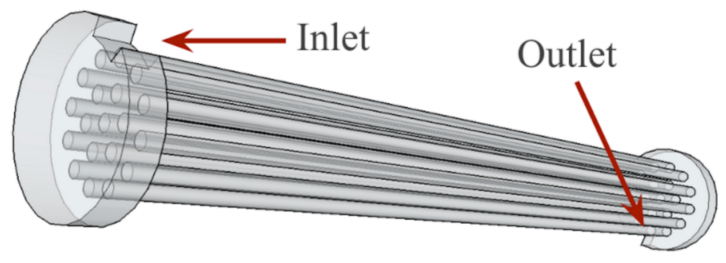

b)

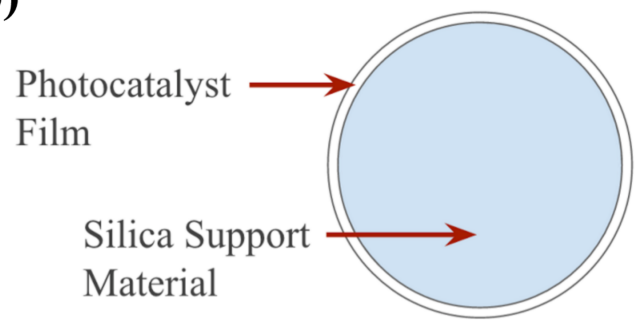

Figure 14: a) simplified 3D model of the photocatalyst cartridge design; b) cross section of a single rod from the cartridge showing the photocatalyst film that has been coated onto a UV-transparent glass rod (not to scale).

A small inlet port was made on end of the cartridge to allow water to enter the reaction chamber; the outlet port, on the opposite end of the cartridge is rotated $180^{\circ}$ with respect 
to the inlet in order to encourage turbulent mixing of the solution within the reaction chamber and to improve mass transfer of pollutant molecules to the catalyst surface.

Notice that as the rod diameter increases, the ratio of catalyst surface area to water volume also increases. This is due to increased reactor volume being occupied by the glass rods. This can be seen clearly in Equation (38), which was derived by geometrical assessment.

$$
S: V=\frac{38 \pi r_{r} R t}{\pi r_{t}^{2}-19 \pi r_{r}^{2}}
$$

Here, $r_{r}$ is the radius of the rods, $r_{t}$ is the radius of the reaction tube, $R$ is the material roughness factor (approximately $118 \mu \mathrm{m}^{-1}[56]$ ), and $\mathrm{t}$ is the thickness of the photocatalyst film. This is why it is crucial to use a highly UV transparent catalyst support: any light absorption that occurs in this volume will not result in generation of oxidizing species due to the insulating properties of silica.

Therefore, the thickness of photocatalyst film should be optimized to provide the maximum photonic efficiency for the degradation reaction, as was described in great detail in Chapter 2. Too thick of a film creates strong light gradients, which are undesirable as this decreases uniformity of the light distribution within the reactor. And if the films are too thin, there will not be enough photocatalyst to absorb all of the light. This will decrease the overall efficiency of the reactor since light that is transmitted through the photocatalyst will be absorbed by the reactor housing and not contribute to the reaction. 


\subsection{Symmetry Alignment with Illumination System}

Since the goal of this device is to create a highly efficient reactor, the choice of equipment for the illumination system is very relevant. It is important to note that this design utilizes LEDs to generate the UV light rather the more standard mercury bulb for several reasons. First, the use of LEDs gives complete geometric control over the illumination geometry, whereas bulb type emitters require the use of mirrors to direct the light, which is limited and inefficient. Second, using LED emitters allows one to choose a particular wavelength of light that is most efficient for the reaction. The ideal wavelength depends on the specific materials used for the photocatalyst and photocatalyst support. Third, the efficiency of the process for generating light from electricity is better for LEDs than for mercury bulbs (at least for some UV-A type LEDs), and continues to improve as the technology evolves.

However, the defining aspect of the geometrical design is the implementation of a correspondence between the rotational symmetry of the illumination system and the catalyst material, shown in Figure 15. This combination improves the conversion efficiency of UV photons to oxidative species. Specifically, the illumination-catalyst system is symmetrically aligned such that: 1) the arrangement of UV emitters has a degree of cylindrical symmetry; 2) the LEDs focus on the axis of symmetry for the system; 3) the geometrical shape of the photocatalyst has a degree of rotational symmetry that matches that on the illumination symmetry; and 4) the center of photocatalyst material is at the focus of illumination. 

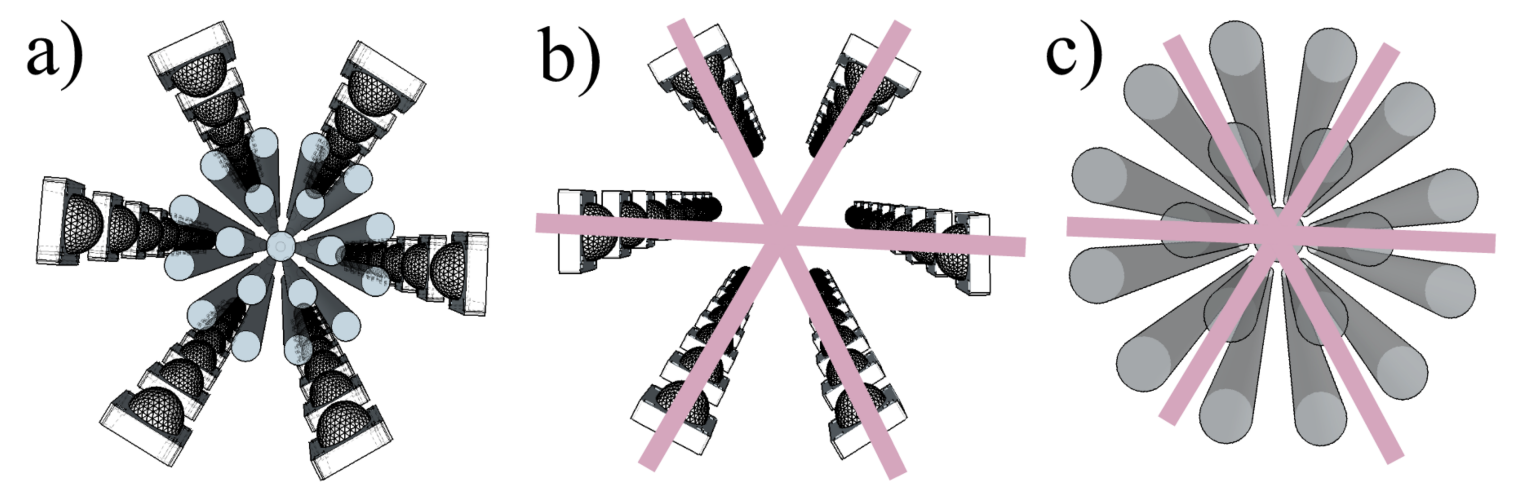

Figure 15: Views of the catalyst material and illumination system from a point on the cylindrical axis of the system to show symmetry alignment. a) catalyst and LED strips together, b) LED strips and lines of rotational symmetry, c) catalyst material and lines of rotational symmetry.

The design of this reactor produces this effect through geometrical arrangement of rods such that each rod (aside from the center rod) is fully illuminated by one strip of LEDs, as shown in Figure 16. This was accomplished by creating 2 concentric rings of rods, with a single rod at their center. The outside ring contains 12 rods, and is anti-aligned with the LED strip such that a line connecting two opposing LEDs runs directly between two of the rods in the outer row. Conversely, each of the 6 rods of the inner ring lies directly on one of these connecting lines. Therefore, the rotational symmetry of the cartridge corresponds with that of the rotational symmetry of the illumination system, and each row of LEDs is primarily responsible for illuminating 3 of the 18 outer rods.

It is useful to imagine grouping the bundle accordingly, so that it is divided into 6 sections corresponding to the 6-fold symmetry of the system. Each section contains an equal portion of the rods. And no individual rods are shadowed from the LED strip that is dedicated to its section. Photons from this LED strip will also be absorbed by rods elsewhere within the bundle, though to a much lesser degree, since all other rods are at 
peripheral angles where emission intensity is relatively small, or they are shadowed by the primary rods.

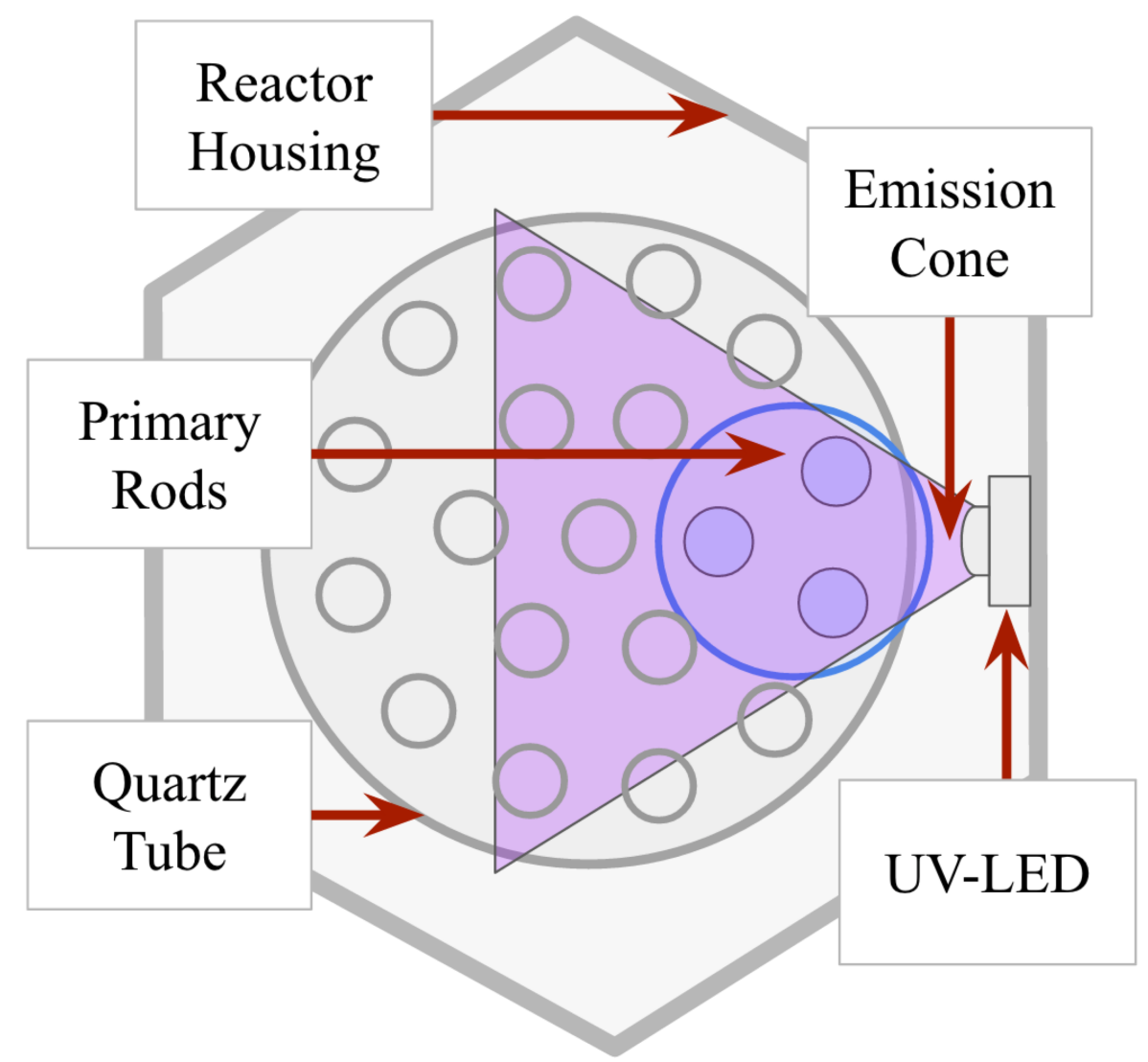

Figure 16: Illustrated cross-section of the reaction chamber showing only one UV-LED in order to emphasize the illumination of the 3 primary rods that are in the direct path of its emission cone.

The central rod, though heavily shadowed, will still receive significant light intensity since it is located at the focus, and will receive a large amount of both scattered and transmitted light. Additionally, its inclusion does not break the 6-fold symmetry of the system, despite the fact that it does not distinctly lie in any particular section. 
The purpose of this design is to maintain an approximately even light distribution across all photocatalytic surfaces, allowing the reactor to operate at its maximum efficiency since the efficiency of the overall photocatalytic reaction is actually an average of the efficiencies of the individual constituents of the reaction. The correspondence between the shapes of catalyst and illumination system plays a vital role in increasing the efficiency of the reactor because the conversion of photons to oxidizing species at any particular point on the catalyst film is a function of light intensity, as shown in Chapter 1.

\subsection{Light Path Optimization}

Another very important quality of the catalyst bundle is that its geometrical shape does not contain any direct paths from the emission point of any LED through the catalyst bundle, such that the path does not intersect the surface of at least one rod. This design ensures that light is not passing through the bundle without interacting with the photocatalyst material, since any light that doesn't interact is wasted, causing a decrease in system efficiency.

Careful examination of the images shown in Figure 17 emphasizes this point by showing the catalyst bundle from a reference point on one of the lines of symmetry (where an LED would be located). From these images, it can be seen that there are no holes in the catalyst material for light to pass through. That is, all light emitted from an LED must be incident on a catalyst surface at least once. 

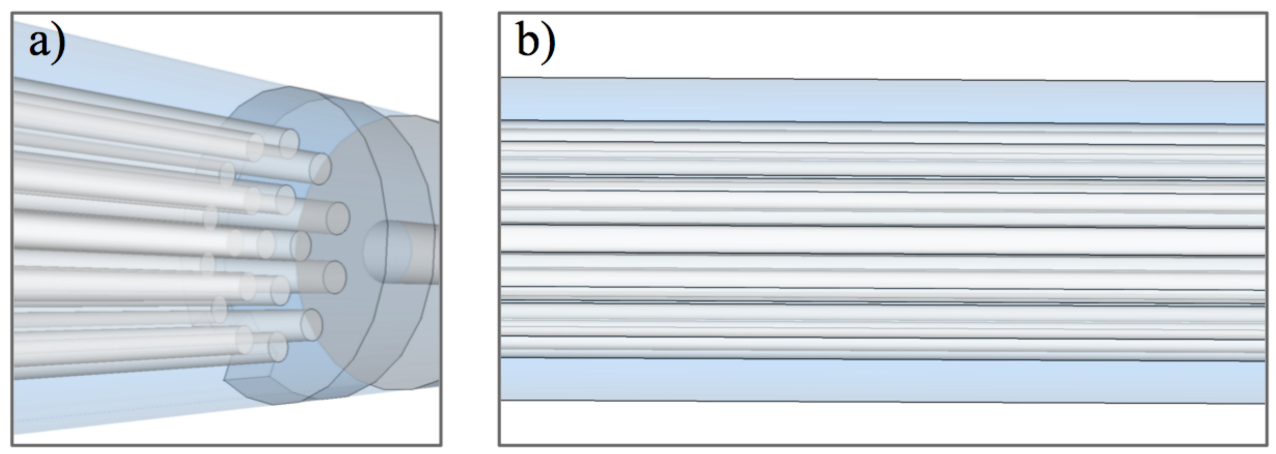

Figure 17: Views of the catalyst material from the position of an LED. View a) shows the insertion points of the rods into the holder for reference. View b) is shown to elucidate the fact that there are not optical holes in the catalyst material from the perspective of the illumination source.

To clarify this point, Figure 18 shows the catalyst bundle from an orientation that is not on one of the lines of symmetry. From this perspective, it is clear there do exist optical paths, or "holes" in the catalyst bundle. If the LEDs were to be mounted at this angle, light would pass through these holes and likely be absorbed by the reactor housing, essentially causing a loss of energy and system efficiency. Yet the use of LEDs for the reactor provides a discrete set of illumination points, allowing these losses to be avoided.

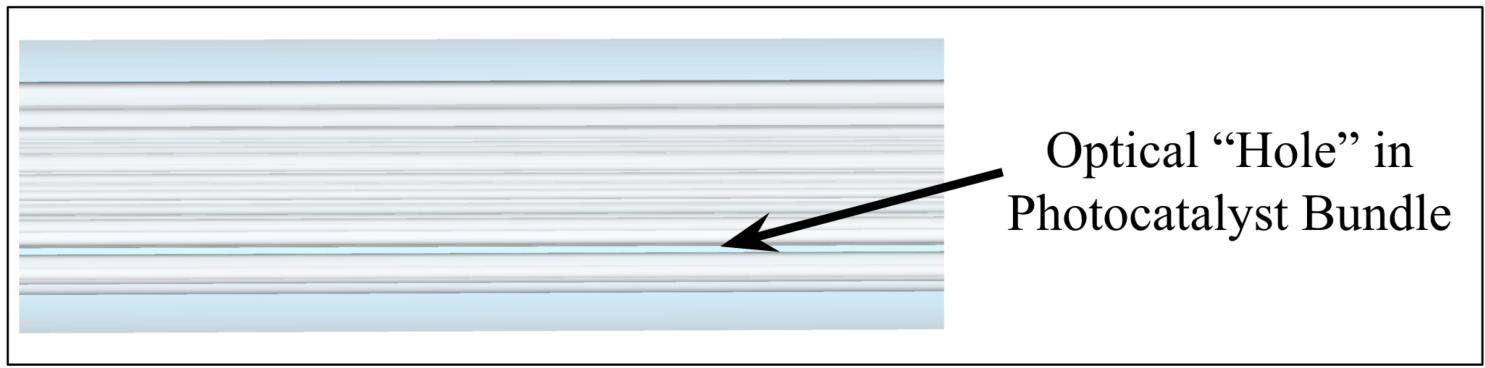

Figure 18: View of the catalyst material from the perspective of an LED when the rotational symmetry of the catalyst and illumination system are misaligned by $5^{\circ}$, resulting in a "hole" such that light can pass completely through the $3 D$ material without interaction.

This arrangement has the additional benefit of not having any direct line-of-site from an LED to the reactor wall except for a very low percentage $(3 \%)$ of light that leaves the 
emitter at a high angle ( $>65^{\circ}$ from center). That is, $97 \%$ of the photons emitted from a given LED must be incident on the photocatalyst surface at least once. Furthermore, this design provides the opportunity for most photons that are transmitted or reflected by the photocatalyst film to be absorbed at another photocatalyst surface, increasing the system efficiency. The extent of this effect can be modified by manipulation of the geometrical parameters, such as the total number of rods, inter-rod spacing, tube and rod diameter, photocatalyst thickness, etc.

\subsection{Application of the Theoretical Model}

The ideal output of the theoretical model presented in Chapter 1 was considered to be quantitative optimization of the photonic efficiency for the chosen 3D material. However, the purely mathematical approach of the theory does not lend itself well to deviation from planar symmetry. The primary difference between this geometrically complex design and the simple models from Chapter 1, is the formulation of the generation function $\mathrm{g}(\mathrm{x})$.

For instance, consider just a single rod from the bundle, as shown in Figure 19, where a photocatalyst material is coated onto a cylindrical support. The reflection, refraction, and scattering of the light as it interacts with this geometry dissolves the validity of the absorption plane concept from Section 2.2, making formulation of the intensity at a given point, $\mathrm{p}$, significant more intensive than for the planar case. To then consider the entire 3D geometry including all 19 rods makes the problem too cumbersome to be dealt with by pure mathematics. 


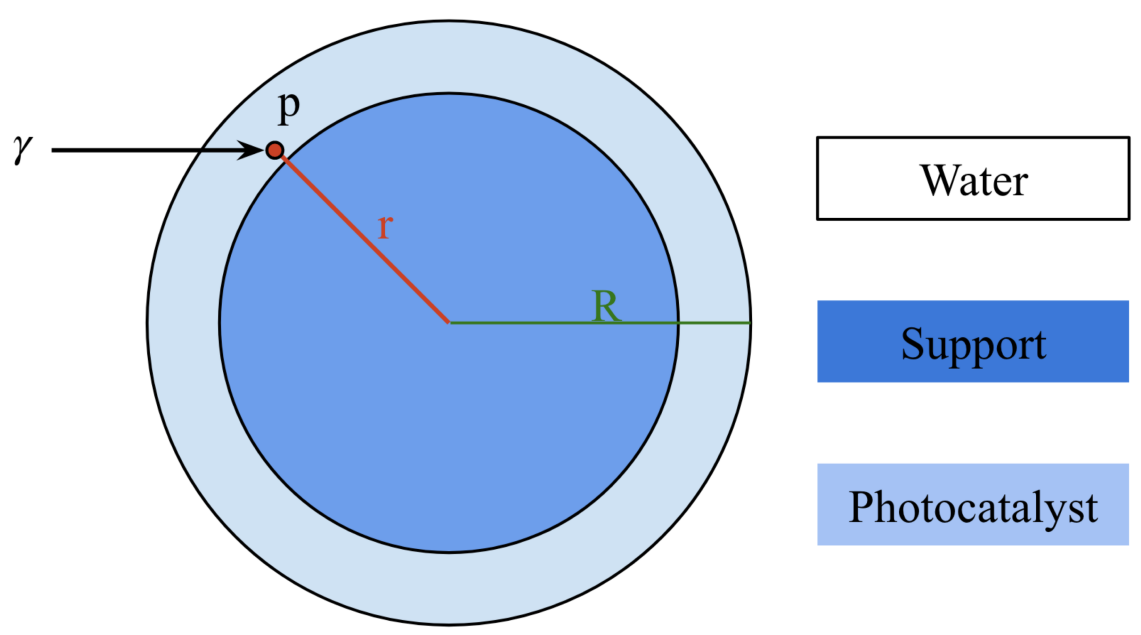

Figure 19: Schematic diagram for the adaptation of the theoretical model to the material design.

However, if the optical properties of the materials being used are known, the $\mathrm{g}(\mathrm{x})$ function can be calculated numerically by either finite element analysis or by a stochastic methodology such as a Monte Carlo simulation. In this case, the generating function will become a scalar field that is a function of both radius, $r$, and polar angle, ${ }^{-1}: g(r, \quad)$ The surfacing function, on the other hand, can still easily be calculated by a single coordinate since the distance of the point $p$ from the catalytic surface can be found through the quantity, $r^{\prime}$, where $r^{\prime}=R-r$ with $R$ being the fixed radius of the rod, and $r$ is the radial distance of point $\mathrm{p}$ from the center of the rod: $s\left(r^{\prime}\right)$.

Simulation by this approach is currently in consideration using the finite element software COMSOL Multiphysics with the additional capability of the software's Wave Optics module. Although a precise quantitative theoretical optimization of the $3 \mathrm{D}$ structure is yet incomplete, assessment of the 13-layer system results from Section 2.5.2 allows for 1st order approximation of the ideal film thickness. The relationship between 
the actual geometry and the 13-layer system is apparent when one considers a crosssectional plane that cuts through the reaction chamber. The orientation of this plane is defined by the fact that it simultaneously bisects two opposing LED strips, such that the cylindrical axis of the reaction chamber lies on the plane. Any one of the six possible planes that satisfy these conditions will intersect 13 material layers in a similar fashion to the 13-layer system for which $\xi(t)$ was calculated and found to have an optimal photocatalyst thickness of $\sim 600 \mathrm{~nm}$. Though this is a rough approximation, it is sufficient to allow to begin empirical experimentation. 


\section{Chapter 4: Experimentation}

The photoreactor described in Chapter 3 was built and tested for its efficiency. There were many stages of development that were required to accomplish this task. This chapter describes the procedures, methods, and qualitative results that were used in order to develop the photoreactor system with the degree of control required to validate the design concepts through quantitative measurement of system efficiency. The work consisted of 1) synthesis and refinement of photocatalyst thin-film, 2) application of the $\mathrm{TiO}_{2}$ coatings to the 3D cartridge design, 3) engineering of the photoreactor system, and 4) design and engineering of efficiency analysis method.

\subsection{Photocatalyst Thin-Film Synthesis}

\subsubsection{Preliminary Results}

The uniformity of the coatings is vitally important to the validity of this research since one of the primary assumptions used in the theoretical development is that the films are of consistent thickness. For this reason, it was necessary to use a very accurate and precise method for coating the fused silica substrates, and since applicability of this research is one of the primary aspirations of this work, the method should not be cost prohibitive. Since dip coating meets all requirements, it was evaluated for uniformity, photoactivity, and durability.

Preliminary testing was done using a $\mathrm{TiO}_{2}$ nanoparticle immobilization method. Because it is scalable and low cost, P25 is a tempting choice as a raw material. As a ceramic 
nanoparticulate powder, it is quite simple to create coatings or even 3D materials from P25 powder. Thus, many researchers that have utilized this technique because of its wellestablished photoactivity.

Coatings were created by preparing a solution of Degussa P25 particles in aqueous solution and dipping the silica substrates into the solution. After many trials to find the proper solution concentration and investigate the use of stabilizers and binders, highly uniform thin-films were created. However, as can be seen in Figure 20, the coatings do not adhere well to the silica substrates; they can be very easily removed just by wiping the glass surface with a finger. The adhesion is improved slightly after high-temperature treatment above $800^{\circ} \mathrm{C}$ [57]. However, heat treatment above $500^{\circ} \mathrm{C}$ results in conversion of the $\mathrm{TiO}_{2}$ crystal phase from anatase to rutile, and nearly all photocatalytic activity is quenched after treatment at $800^{\circ} \mathrm{C}[58]$. Therefore, this technique was abandoned due its inability to produce durable coatings.
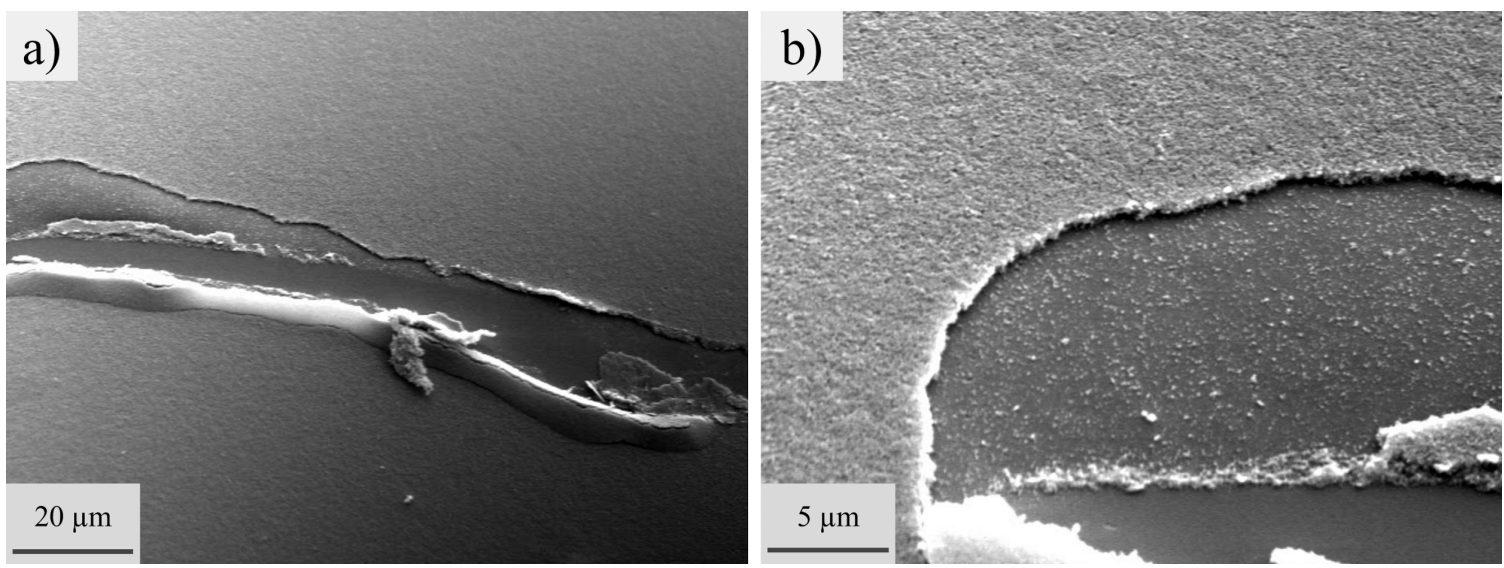

Figure 20: Initial coating attempts using immobilized $\mathrm{TiO}_{2}$ nanoparticles; though the coatings are uniform and highly photoactive, they have poor adhesion the substrate. 


\subsubsection{Sol-Gel Dip Coating Method}

To improve the coating durability, a technique was developed to coat the catalyst support substrate using a modified sol-gel processing method. Sol-gel processes are defined by the use of a metal alkoxide solution, which is a liquid organic reagent that can-mixed with alcohol (and sometimes water) to form a viscous suspension of the metallic atoms [61]. The sol-gel can then be coated onto the substrate. Heat treatment of the substrate then results in combustion of the organic constituents, leaving a dense film of oxidized metal atoms.

Thus, a titanium alkoxide sol-gel was prepared and coated onto the fused silica substrates using a dip coating procedure. This method is a modified version of a method developed by Tada et. al. for stabilizing the alkoxide, titanium tetra-isopropoxide (TTIP), with acetylacetone (AcAc) [54, 59]. The method itself is based on the work of Shinmou [60] for synthesis of $\mathrm{ZrO}_{2}$ films. The advantages of this particular synthesis method are as follows: 1) simple and scalable process, 2) thickness control in the range of interest (5 nm - $500 \mathrm{~nm}$ ), 3) strong adhesion to silica substrates, 4) excellent uniformity of coatings, 5) high coating durability, and 6) applicability to both silica rods and slides.

Because the coatings are easier to analyze on flat substrates than on rods, preliminary coating trials and analyses were done on fused silica microscope slides from the supplier as the fused silica rods (Technical Glass Products). This allowed the samples to be compatible with standard analysis equipment sample holders. Once the coating protocol was established using the microscope slides, the same synthesis procedure was applied to the rods. 
The general procedure used to coat the rods was as follows. The fused silica substrates were first cleaned with ethanol and isopropyl alcohol, then sonicated in a solution of ammonium hydroxide ( $\mathrm{pH}$ 13.7) for 10 min to increase the density of hydroxyl groups on the silica surface. This process increases adhesion of the sol-gel to the silica. A sol-gel solution was then prepared by mixing TTIP with acetylacetone (AcAc), followed by an addition of ethanol (EtOH) to adjust the viscosity. Various ratios of TTIP, AcAc, and EtOH were tested for the best coating results. The final mixture was then magnetically stirred for $20 \mathrm{~min}$.
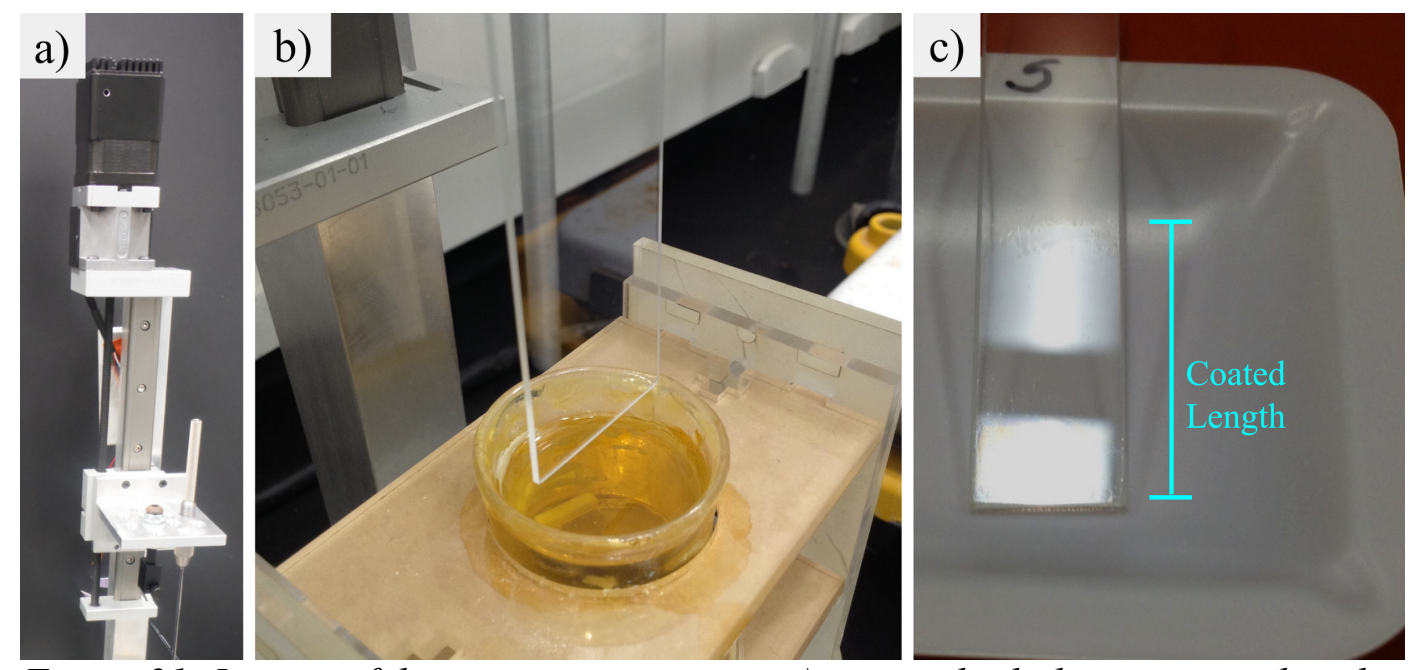

Figure 21: Images of dip-coating apparatus: a) custom built dip-coater with rod and slide attachments, b) a glass slide being dipped into a Ti SG solution, and c) an example of a coated slide.

The silica substrates were loaded into a custom dip-coating apparatus built using a stepper motor and linear bearing to convert the rotational motion of the motor to a smooth and precise linear motion for the dipping process. The dipping rate and dipping length were computer controlled. Two separate attachments were then made to hold the samples in a vertical orientation: one for the rods, and one for slides. The dip-coater and an example of a dipped slide are shown in Figure 21. It was found that both the lowering 
speed of the dip coater and the dwell time in solution had no effect on the resulting coating, but that the withdrawal speed did have a large impact. A withdrawal rate of 2.5 $\mathrm{mm} / \mathrm{s}$ was established as the speed that resulted in the most uniform coatings. After being coated, the substrates were placed into an oven at $60^{\circ} \mathrm{C}$ to remove the ethanol from the coating. After 1 hour, the transparent yellow coatings were dry and well adhered to the substrate. The substrates were then placed in a high-temperature oven to produce $\mathrm{TiO}_{2}$ via calcination at $500^{\circ} \mathrm{C}$ for 2-hours. The result was a visibly transparent, anatase $\mathrm{TiO}_{2}$ film.

\subsubsection{Qualitative Assessment of $\mathrm{TiO}_{2}$ Films}
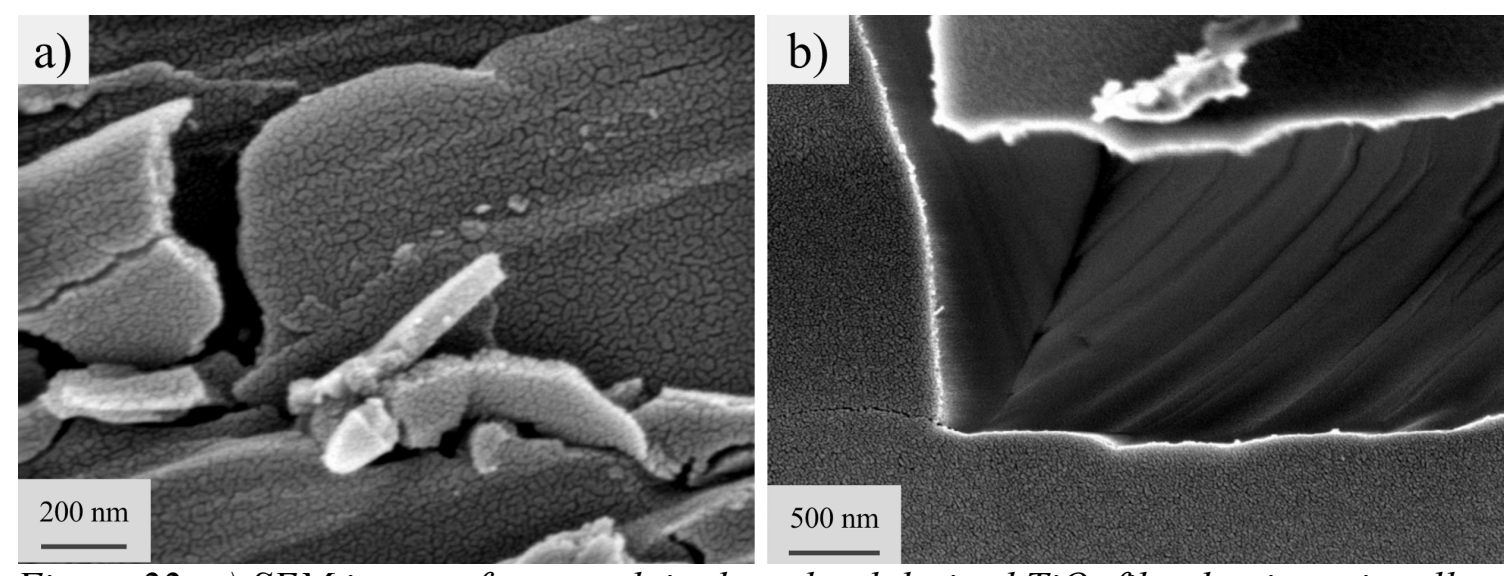

Figure 22: a) SEM image of a scratch in the sol-gel derived $\mathrm{TiO}_{2}$ film that intentionally created to analyze the thickness and composition of the film; b) SEM image of a location on cracked film that reveals the silica glass surface underneath.

An example of a sol-gel derived film is shown in Figure $\mathbf{2 2}$ for direct comparison to the immobilized P25 films. Both Figure 20 and Figure 22 show areas of the films that were intentionally marred with a diamond scribe for analysis purposes. As can be seen from these images, the sol-gel derived films are hard and durable. The area that was scratched is cracked or even shattered, indicating the rigidity and brittleness of a ceramic material. This is clearly not the case with the immobilized p25 films which appear to be compacted 
particles that easily crumble and pull away from the silica. A broad range of film thicknesses were then created to determine the effect on film uniformity and durability. The primary factor of consideration was the prevention of crack formation, as this reduces the durability of the film and is unacceptable for application purposes. The synthesis parameter found to have the greatest impact on the formation of cracks was the viscosity of the dipping solution. This is due to the fact that the viscosity of the solution is directly correlated with the thickness of the coating and thicker films have greater tendency to crack due to capillary pressure created during the drying process [61].

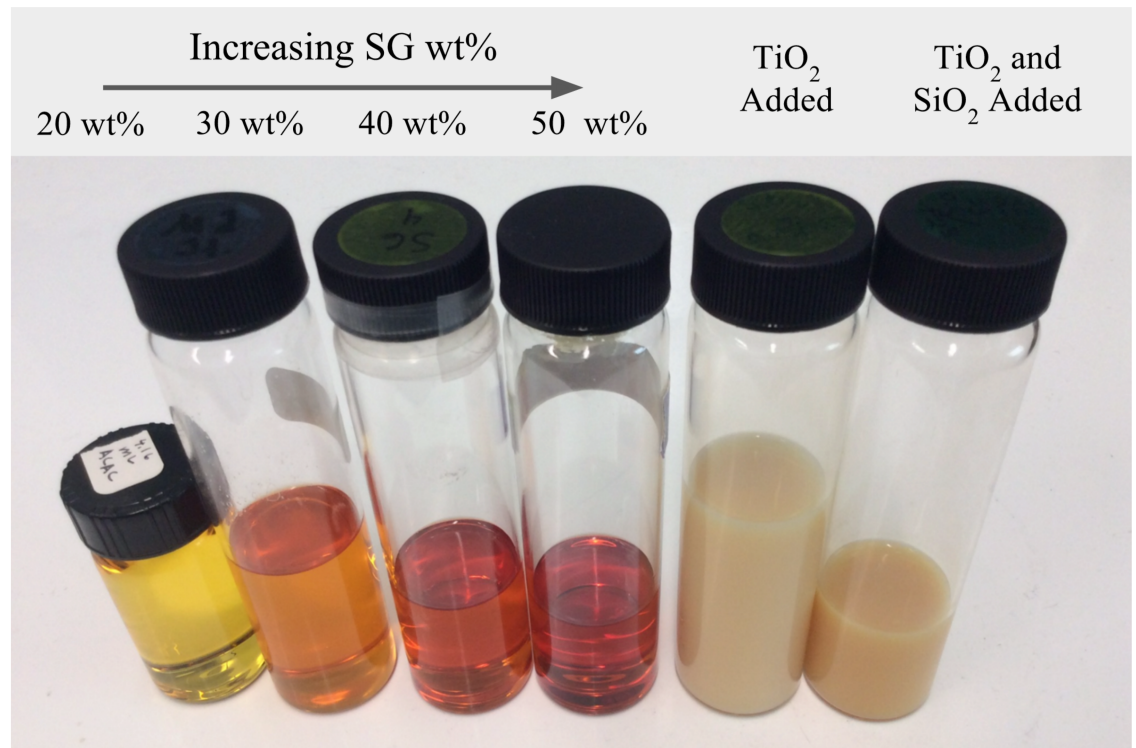

Figure 23: As the concentration of $S G$ in the dipping solution increases, the color changes from yellow (20 wt\%) to red (50 wt\%). This change also results in an increasing solution viscosity. When nanoparticulate $\mathrm{TiO}_{2}$ and $\mathrm{SiO}_{2}$ is added, the solution the becomes opaque. The two samples on the right were made using a 20 wt $\%$ solution with $0.30 \mathrm{wt} \% \mathrm{TiO}_{2}$ and $0.30 \mathrm{wt} \% \mathrm{TiO}_{2}+0.05 \mathrm{wt} \% \mathrm{SiO}_{2}$ respectively.

The viscosity of the solution was adjusted by diluting the sol-gel stock with ethanol; increasing the amount of ethanol led to less viscous solutions and thinner films. Figure 24 shows this effect clearly: as the SG wt $\%$ of the solution is increased, the density of 
cracks also increases. Because the cracked films were not considered viable for implementation, the practical range of SG concentration was determined to be $5-30 \mathrm{wt} \%$.
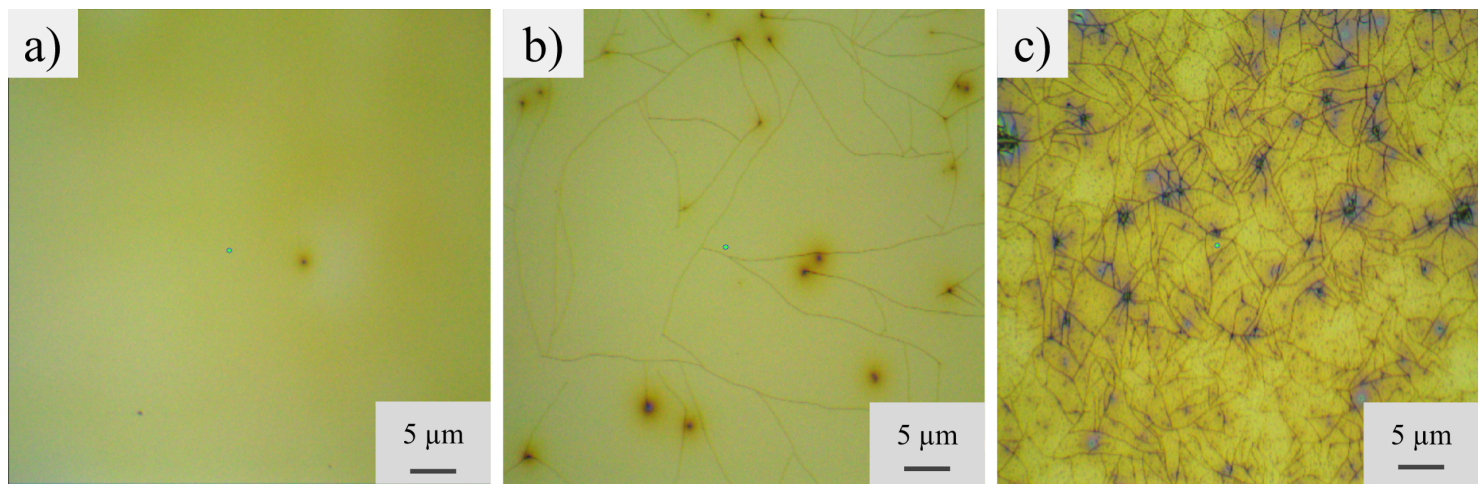

Figure 24: Optical images of SG films prepared with increasing solution viscosity by increasing the concentration of Ti precursor: a) $30 \mathrm{wt} \%$, b) $40 \mathrm{wt} \%$, c) and $50 \mathrm{wt} \%$.

Removal of contaminants from the silica surface was also an important factor for creating crack free films. The SEM images in Figure 25 shows how particulate matter creates breaks in the film. Cleaning substrates using sonication in ethanol and 2-propanol, followed by a DI water rinse, then sonication in ammonium hydroxide $(\mathrm{pH} 13.7)$ resulted in nearly clean films. However, this method was not able to produce completely defectfree films. Therefore, refinement of the cleaning process is still required for pristine films. Nevertheless, these defects were quite useful for determination of the film thickness and for microscopic analysis of the film composition.
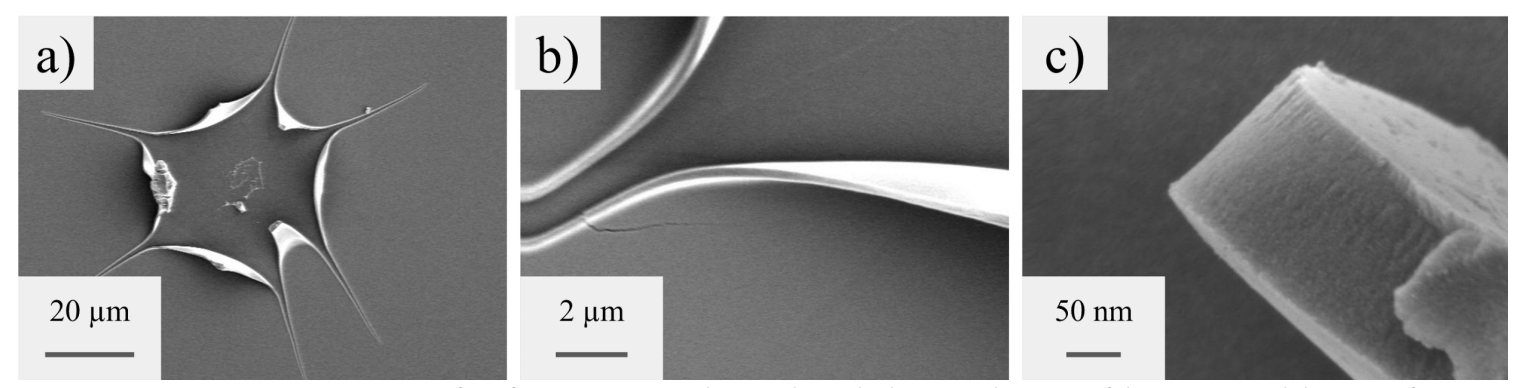

Figure 25: SEM images of a fracture in the sol-gel derived $\mathrm{TiO}_{2}$ film caused by surface contamination, acquired at a) $\sim 2,500 X, b) \sim 18,000 X$, and c) $\sim 260,000 X$. 


\subsubsection{Nanoparticle Implantation}

Modifications to the sol-gel solution were then made in order to increase the

photocatalytic activity of the film by incorporating nanoparticulate $\mathrm{TiO}_{2}$ and $\mathrm{SiO}_{2}$ into the dipping solution (Figure 23). The inclusion of both types of particles created more catalyst surface area by increasing both porosity and surface roughness.

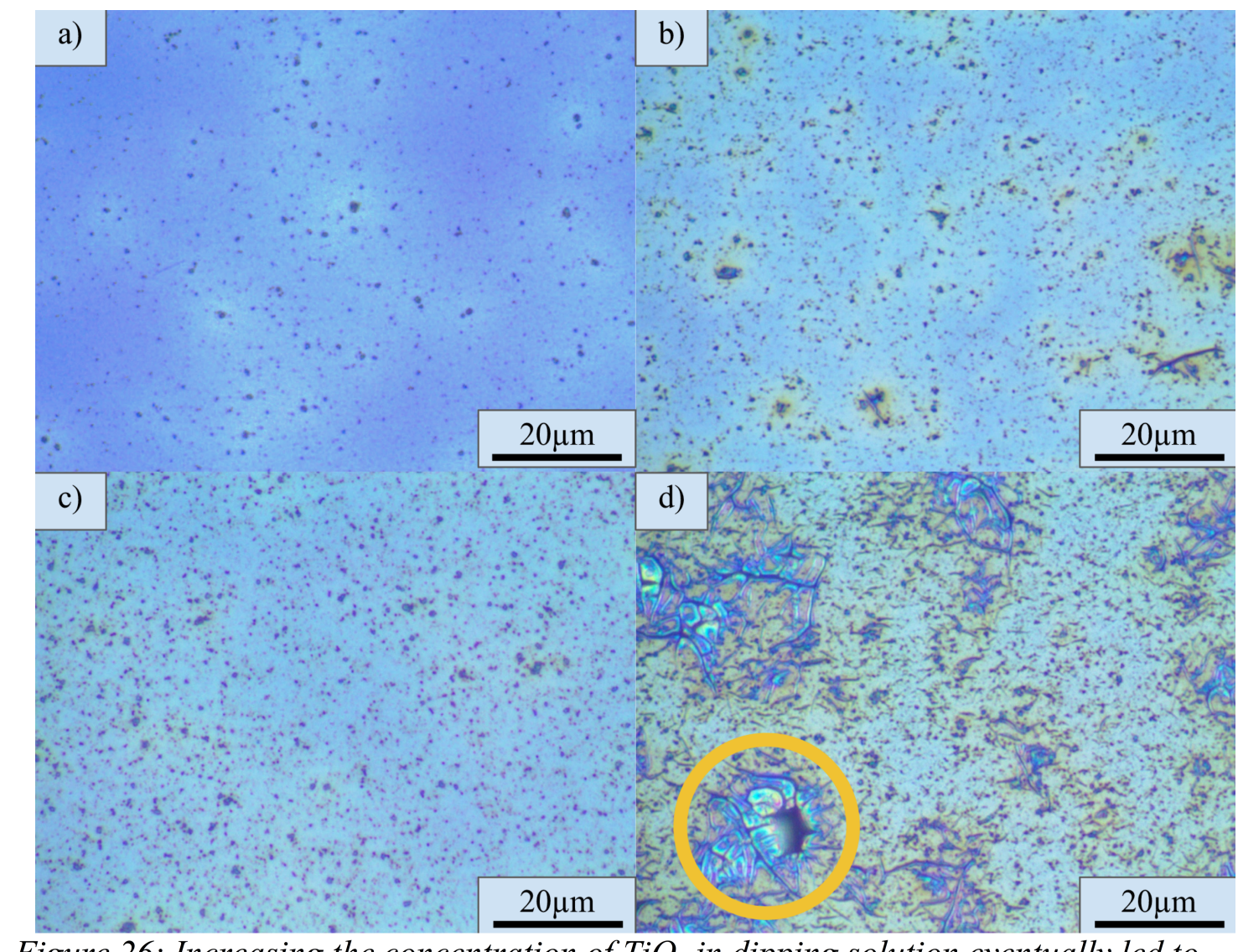

Figure 26: Increasing the concentration of $\mathrm{TiO}_{2}$ in dipping solution eventually led to crack formation: a) $0.10 \mathrm{wt} \% \mathrm{TiO}_{2}$; b) $0.20 \mathrm{wt} \% \mathrm{TiO}_{2}$, c) $0.30 \mathrm{wt} \% \mathrm{TiO}_{2}$, d) $0.40 \mathrm{wt} \% \mathrm{TiO}_{2}$.

Additionally, The $\mathrm{TiO}_{2}$ particles act as crystal growth seeds, encouraging the dispersed growth of both anatase and rutile phases within the film, while the $\mathrm{SiO}_{2}$ particles are added to increase pollutant adsorption and decrease electron-hole pair recombination by 
acting as hole traps: both of which have been shown to increase photodegradation rates $[49,62]$.

To begin experimentation, a $5.0 \mathrm{wt} \%$ dipping solution was chosen for a baseline recipe, which was modified by mixing nanoparticles into the base solution using sonication. As with the sol-gel wt $\%$, it was found that there was an upper limit to how much particulate matter could be added before the films began to crack due to thickening of the dipping solution as can be seen in Figure 26. The upper limit for the addition of $\mathrm{TiO}_{2}$ was determined first, and was found to be $0.30 \%$ by weight.
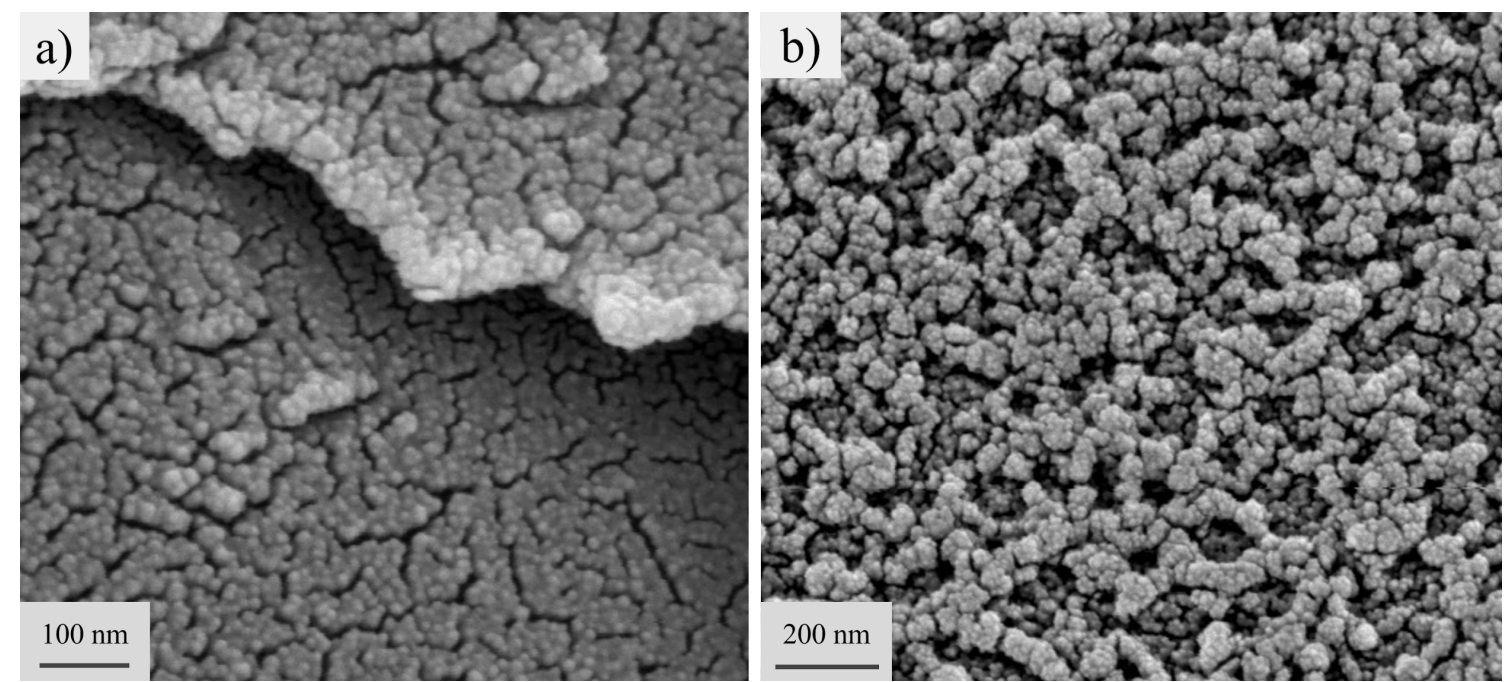

Figure 27: Sol-gel derived films have much more porosity and surface area after adding nanoparticles to the dipping solution: a) $0.30 \mathrm{wt} \% \mathrm{TiO}_{2}$ particles, b) $0.05 \mathrm{wt} \% \mathrm{SiO}_{2}$ particles in addition to $0.30 \mathrm{wt} \% \mathrm{TiO}_{2}$ particles.

This process was then repeated by adding $\mathrm{SiO}_{2}$ particles to the dipping solution.

Similarly, the upper limit for addition of $\mathrm{SiO}_{2}$ was found to be $0.10 \mathrm{wt} \%$ in solutions without $\mathrm{TiO}_{2}$ particles added, and $0.05 \mathrm{wt} \%$ in solutions with $\mathrm{TiO}_{2}$ added. Thus, a final solution was created from a solution of $20 \mathrm{wt} \%$ sol-gel combined with $0.30 \mathrm{wt} \% \mathrm{TiO}_{2}$ and $0.05 \mathrm{wt} \% \mathrm{SiO}_{2}$. The resulting films are shown in Figure 27, which show the increased 
porosity and surface roughness achieved by this technique in comparison to SG only films (Figure 22).

\subsubsection{Material Characterization}
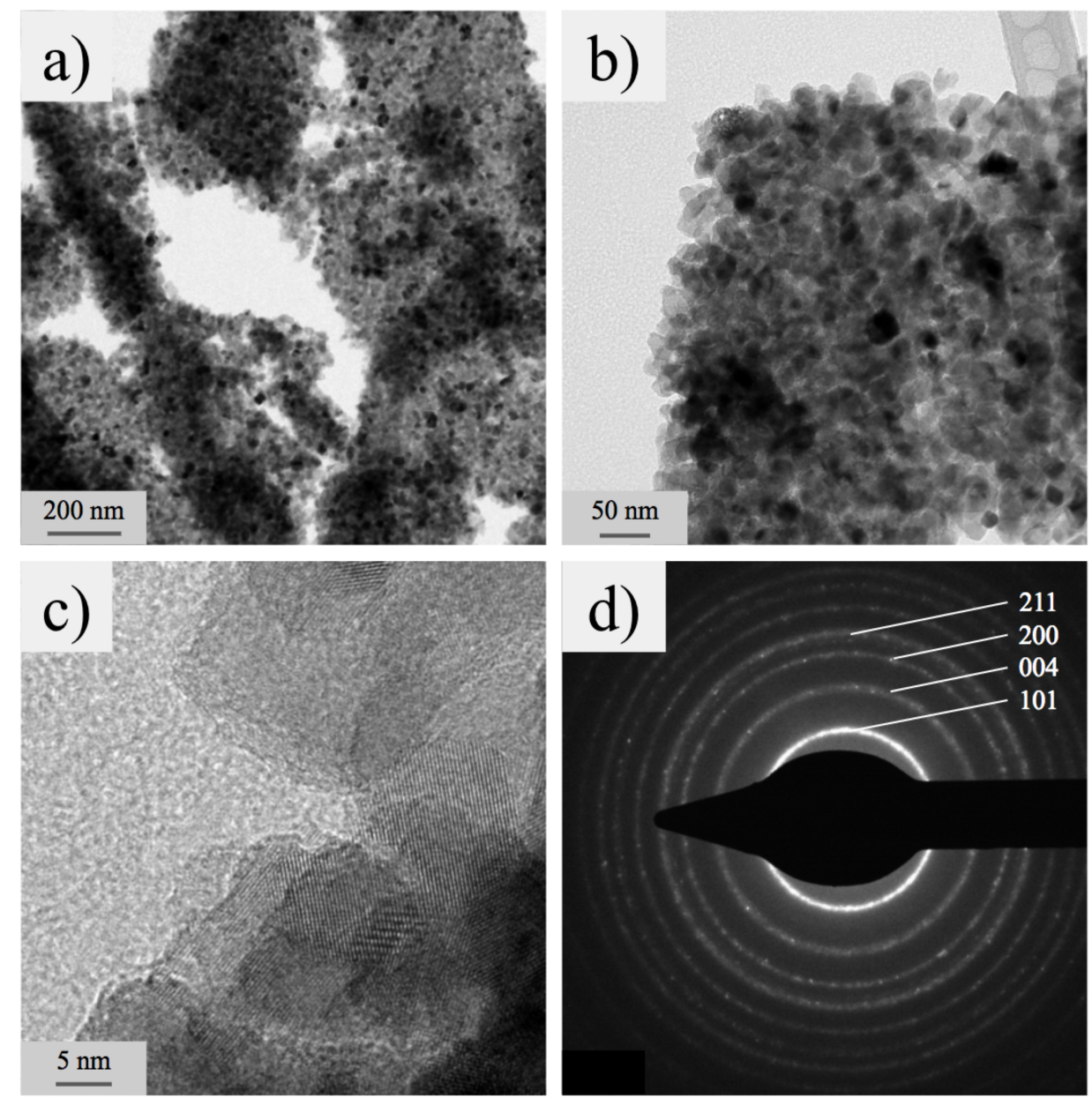

Figure 28: a) and b) are low magnification TEM micrographs of fragments of a sol-gel derived film showing that the film is polycrystalline. This is clearly evident in the HR$T E M$, image c), and by the formation of distinct rings in the electron diffraction pattern, image d). The spacing of the rings in the diffraction pattern confirm that the film is anatase $\mathrm{TiO}_{2}$.

The films were characterized by SEM, TEM, Raman Spectroscopy, and UV-vis

spectroscopy. The analysis shows that the films are, in fact, crystalline $\mathrm{TiO}_{2}$. The TEM micrographs shown in Figure 28 were acquired from a $20 \mathrm{wt} \%$ SG solution (without 
nanoparticle addition). These images show that the after a 2 hour calcination at $500^{\circ} \mathrm{C}$, the films are highly crystalline. The crystals have been identified as anatase $\mathrm{TiO}_{2}$ from the lattice spacing measurements obtained from both the HR-TEM image and electron diffraction pattern.

As described in the Literature Review, both the anatase and rutile phases of $\mathrm{TiO}_{2}$ are effective photocatalysts but the activity is enhanced if both phases are present. This effect is optimal at a ratio of approximately $30-40 \%$ anatase to $70-60 \%$ rutile $[44,45]$. Since temperatures above $500^{\circ} \mathrm{C}$ result in conversion from anatase to rutile phase, this ratio was achieved by heat treating the films at $550^{\circ} \mathrm{C}$ for 3 hours. A representative Raman spectrum collected from these samples is shown in Figure 29 confirming Raman vibrations for both anatase and rutile crystal structures. The phase ratio was calculated to be $74 \%$ anatase and $26 \%$ rutile by Raman spectroscopy using the characterization model presented by Castrejón-Sánchez [63]. A UV-vis absorption spectrum of the same sample is also provided, showing the characteristic $\mathrm{TiO}_{2}$ absorption edge.
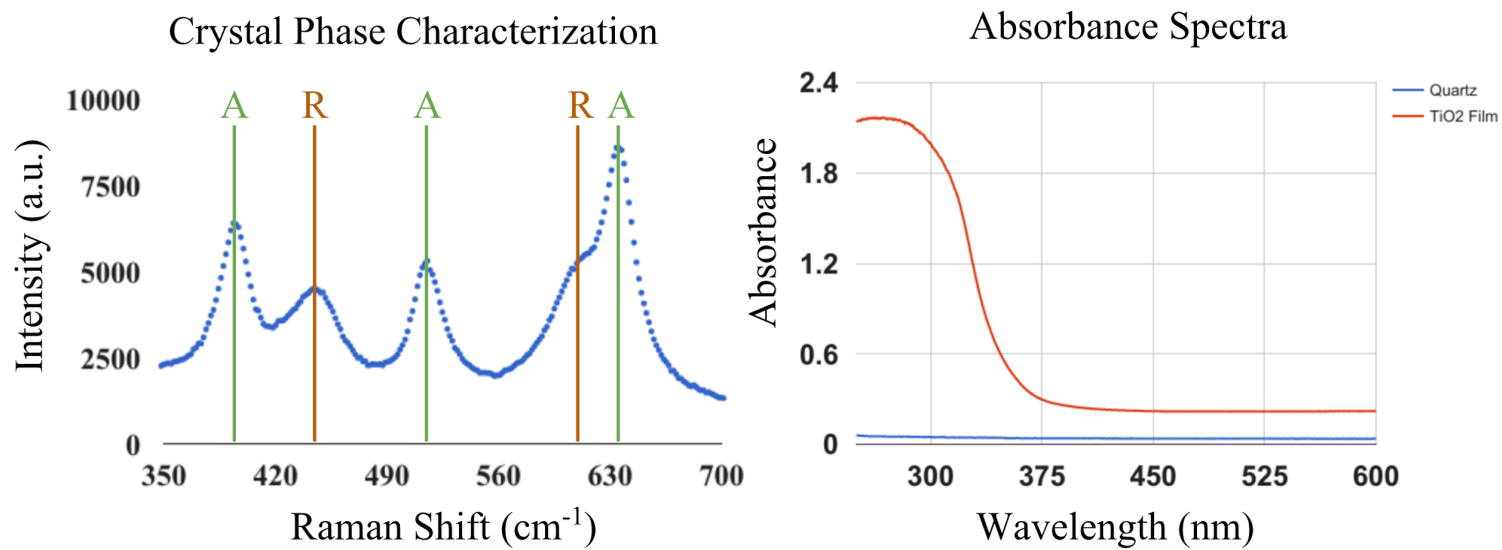

Figure 29: a) Raman spectrum collected sol-gel derived film on quartz slide, showing both rutile and anatase crystalline phases; b) UV-vis spectrum of sol-gel derived film displaying characteristic $\mathrm{TiO}_{2}$ absorption edge in the $U V-A$ range. 
The material band-gap was also determined by UV-vis spectroscopy by the Tauc method [64] and was found to range from 3.4 to $3.2 \mathrm{eV}$ for samples with $0 \%$ and $0.3 \% \mathrm{TiO}_{2}$ particles, respectively, which supports the hypothesis that that rutile growth would be seeded by including P25 particles in the dipping solution. On the other hand, the addition of $\mathrm{SiO}_{2}$ particles had no measurable effect on the band-gap.

The effect of heat treatment on the band gap was also determined. The grain size, and band-gap of samples heat treated at $500^{\circ} \mathrm{C}$ and $1000^{\circ} \mathrm{C}$ is shown in Figure 30. As expected, the grain size increases from higher temperature treatment. The material bandgap also decreases at higher calcination temperatures. This is due to the phase conversion from anatase to rutile as described earlier. The rutile phase is known to have a smaller band gap. As will be seen in Chapter 5, calcination temperatures above $600^{\circ} \mathrm{C}$ have markedly decreased photoactivity.

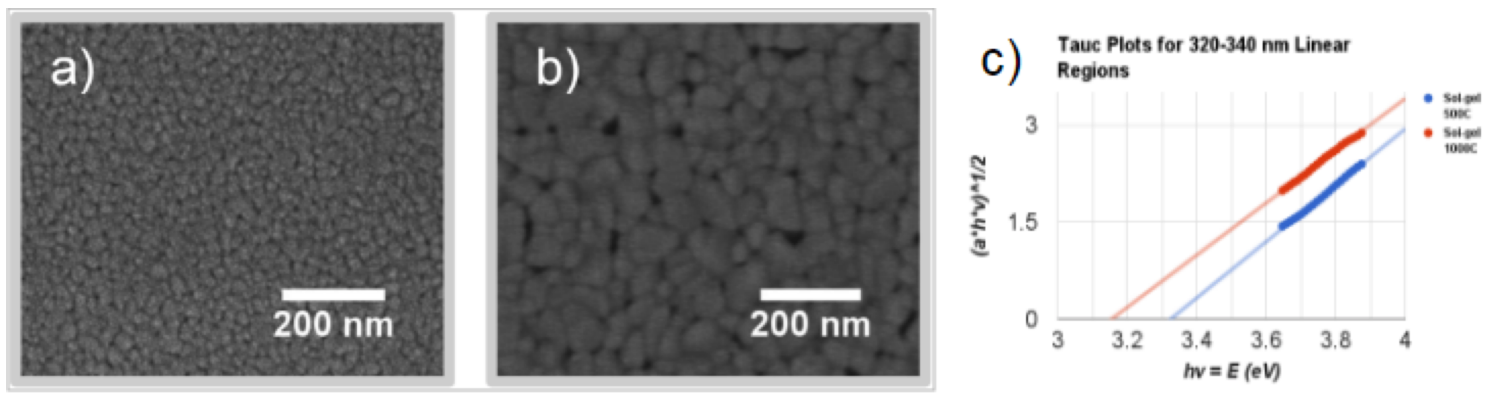

Figure 30: a) SEM image of sol-gel derived coating calcined at $500^{\circ} \mathrm{C}$ for $3 \mathrm{hrs}$; b) SEM image of sol-gel derived coating calcined at $1000^{\circ} \mathrm{C}$ for $3 \mathrm{hrs}$; c) Tauc plots of the $500^{\circ} \mathrm{C}$ and $1000^{\circ} \mathrm{C}$ samples, indicating a reduced band-gap for the $1000^{\circ} \mathrm{C}$ sample.

\subsection{System Engineering and Analysis}

Engineering of the photoreactor was separated into 4 system aspects: 1) the catalyst cartridges, 2) the illumination system, 3) the hydraulic system, and 4) the oxygen sparging system. An analytical system was also designed and built to quantify the 
system's efficiency for degradation of organic markers. This consist of a custom UV-vis spectrometer, which allows real-time monitoring of the marker concentration, as well as a gaseous $\mathrm{CO}_{2}$ monitor to record the amount of $\mathrm{CO}_{2}$ exhausted by the redox reactions.

\subsubsection{Photoreactor Engineering}

A system overview, with each system component identified, is given in Figure 31. In this image system components that make up the photoreactor system are labeled with blue text and components that make up the analytical system are labeled labelled with purple text.

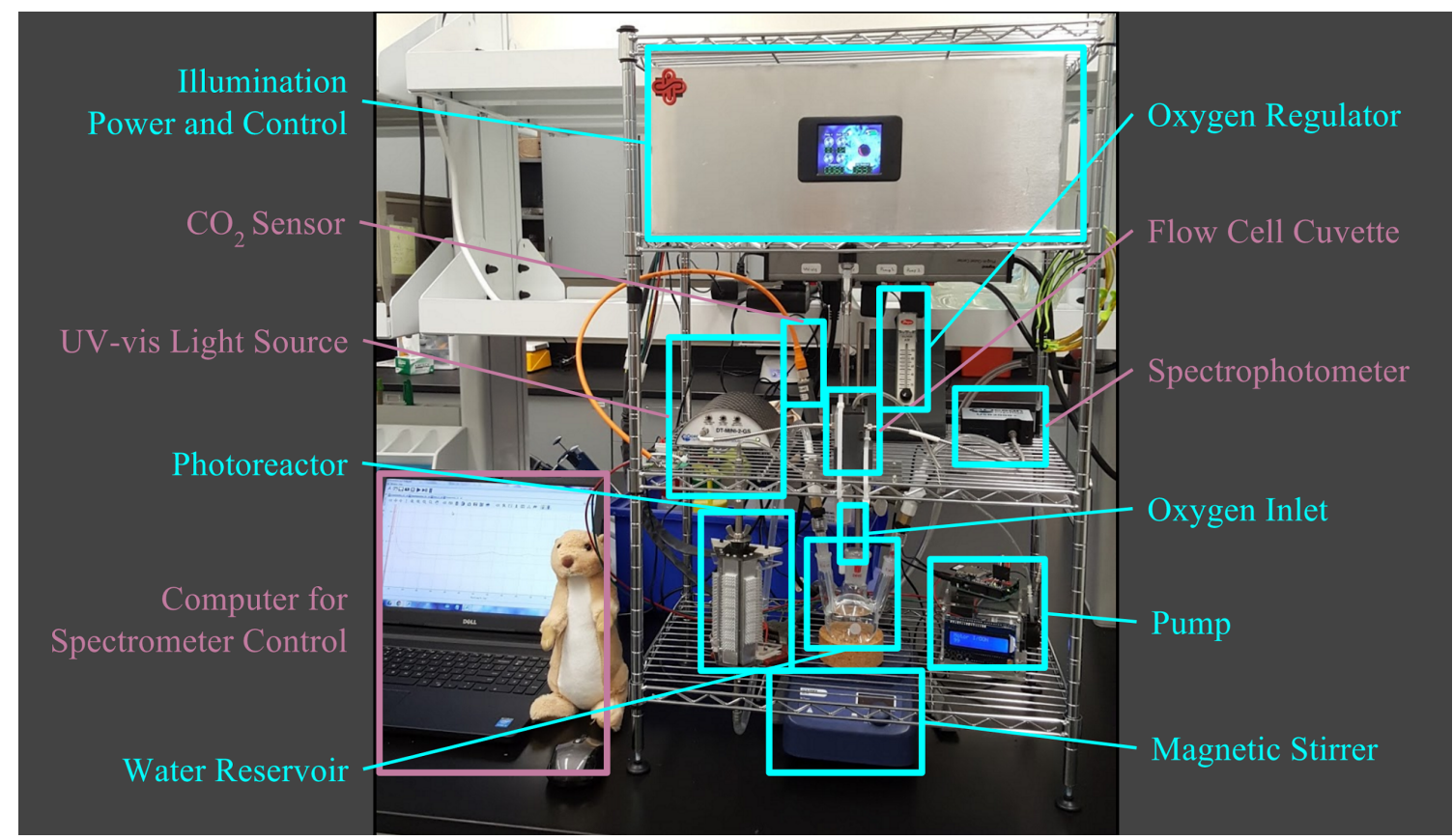

Figure 31: Breakdown of the photoreactor and analysis system components. Photoreactor components are shown in blue and the analysis system components in purple.

\subsubsection{Catalyst Cartridge Fabrication}

In order to fabricate the 3D catalyst cartridges (Figure 32), the synthesis method established on the silica slides was applied to fused silica rods of $1.5 \mathrm{~mm}$ diameter. As before, the rods were cleaned and soaked in ammonium hydroxide solution ( $\mathrm{pH}$ 13.7) to 
increase adhesion of the sol-gel solution to the silica surface. The rods were dried and then dipped into the solution using a withdrawal rate of $6 \mathrm{~mm} / \mathrm{s}$. Twenty rods were coated in an identical manner and placed in a vacuum oven for $1 \mathrm{hr}$ at $60^{\circ} \mathrm{C}$ to remove the ethanol from the coating. The dried films were then calcined at $550^{\circ} \mathrm{C}$ for $3 \mathrm{hrs}$.
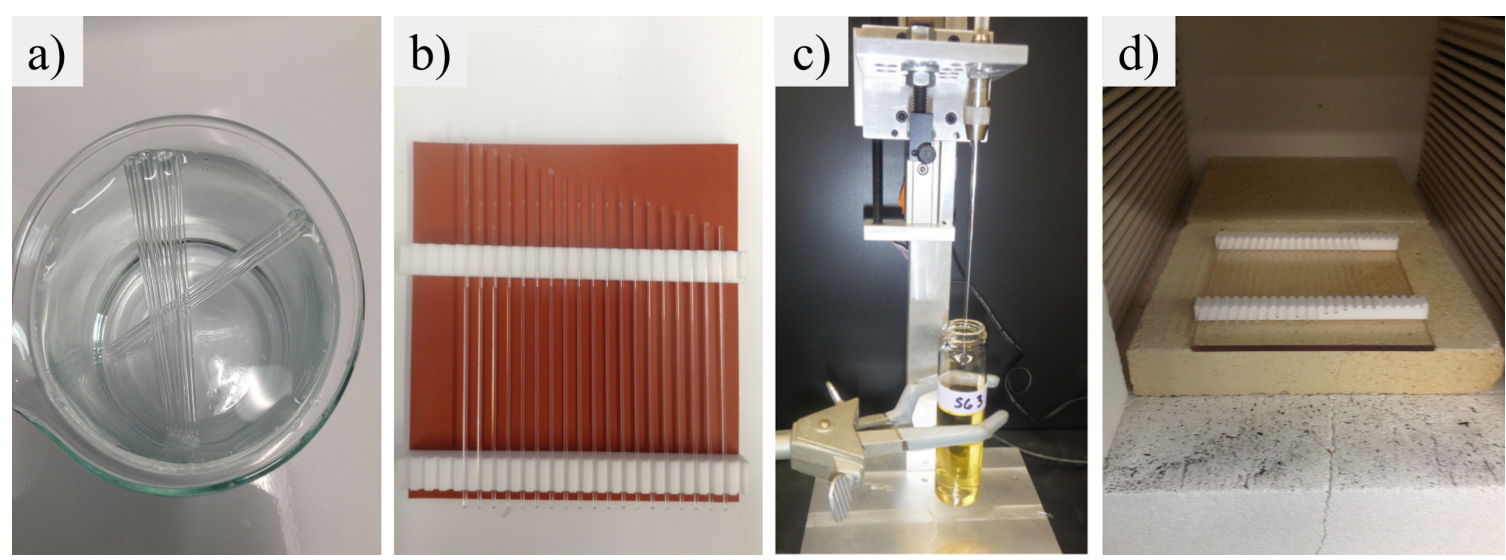

Figure 32: a) Twenty $1.5 \mathrm{~mm}$ silica rods soaking in ammonium hydroxide solution; b) rods are then placed on a custom made drying rack for transfer to vacuum oven to remove all water from the rod surface; c) rods were dipped in sol-gel dipping solution with a withdrawal rate of $6 \mathrm{~mm} / \mathrm{s}$ and returned the vacuum oven to remove ethanol from the coating; d) rods were finally calcined in air at $550^{\circ} \mathrm{C}$ to produce a crystalline $\mathrm{TiO}_{2}$ film.

Once cooled the rods were assembled into the geometrical arrangement shown in Figure

15. The result is shown in Figure 33. This was accomplished by machining stainless steel discs that hold the rods the proper orientation to create the desired 3D geometry. To protect the rods, and especially the rod coating, a silicone rubber gasket was used to interface the steel holders with the rods. This gasket also acts as the reaction chamber inlet and outlet. The diameter of the gasket is slightly larger than those of the steel holders which makes a seal once the assembled cartridge is placed into a quartz tube of $16 \mathrm{~mm}$ inner diameter. Thus, to create a water inlet and outlet holes were cut in the gasket, as were shown in Figure 14. The quartz tube is sealed on both ends using an 


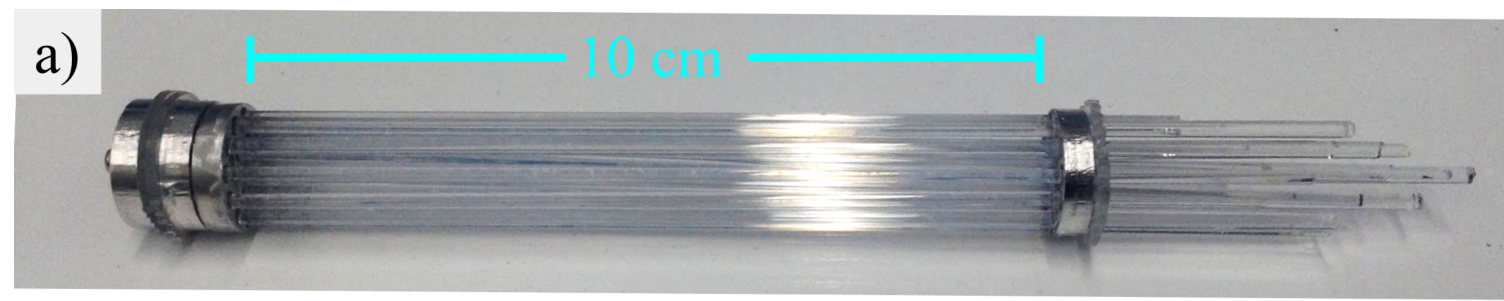

b)
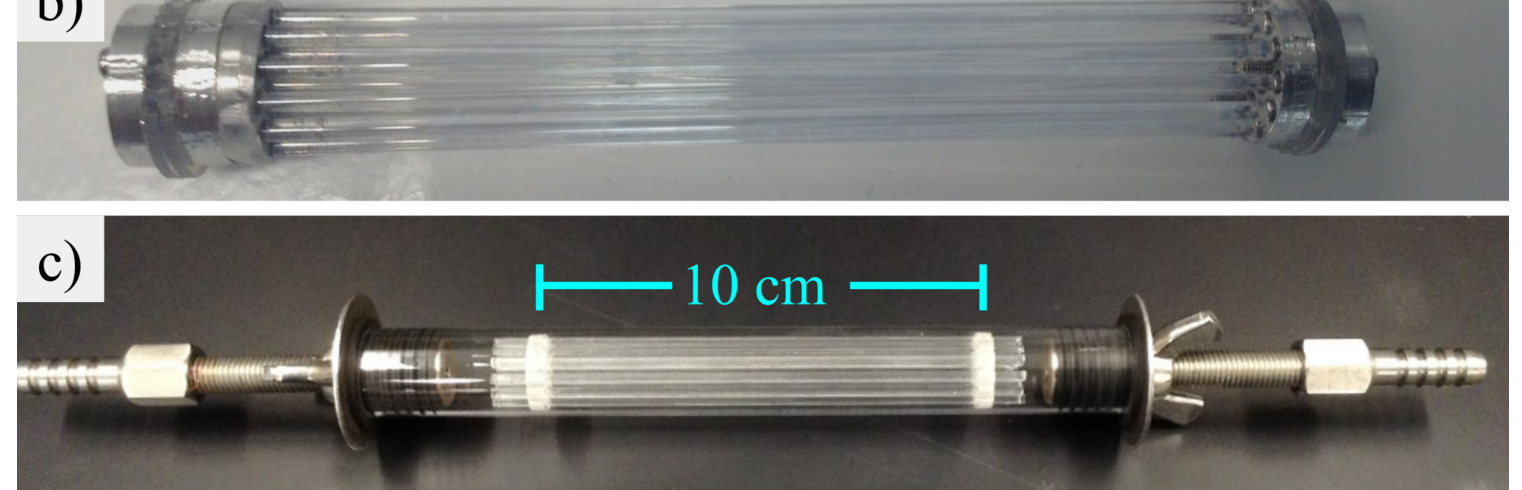

Figure 33: a) the assembly process required building the 3D structure by sliding stainless steel rod holders and a silicone rubber gasket onto each end of rod bundle, leaving a spacing of $10 \mathrm{~cm}$ of active photocatalyst surface; b) the fabrication was finalized by then cutting the excess rod with a diamond saw and capping the the holders with a stainless steel disk; c) the cartridge is then placed into the quartz reaction tube which is sealed on each end with an expansion plug that converts to a barbed hose connector.

expansion gasket system with a water feed-through to barbed hose connector, which connects to the water lines.

\subsubsection{Illumination System}

The desired geometry of the illumination system was shown in Figure 13. The actual system was built using 45 UV-LEDs made by LED Engin (LZ1-30UV00), which have a peak output at $365 \mathrm{~nm}$ and $1200 \mathrm{~mW}$ radiative output at $2.7 \mathrm{~W}$ power dissipation.

Approximately $95 \%$ of the emission is between $355-375 \mathrm{~nm}$ with a full width at half maximum of $10 \mathrm{~nm}$. These characteristics make the chosen LEDs ideal for this system: they have peak emission just above the $\mathrm{TiO}_{2}$ band-gap energy and high power efficiency (44\%). The transmission of these wavelengths by the silica support material and the 
absorption of these wavelengths by the photocatalyst film are vitally important for optimal light harvesting efficiency (as discussed in Chapter 3). Figure 34 shows precisely how the LED emission band relates to both the silica support material and the photocatalyst film. These diagrams clarify two important points: 1) parasitic absorption by silica support material is negligible for a rod of $1.5 \mathrm{~mm}$ diameter; 2) a photocatalyst film prepared from a $20 \mathrm{wt} \%$ sol-gel solution absorbs approximately half of the incoming photons.

a)

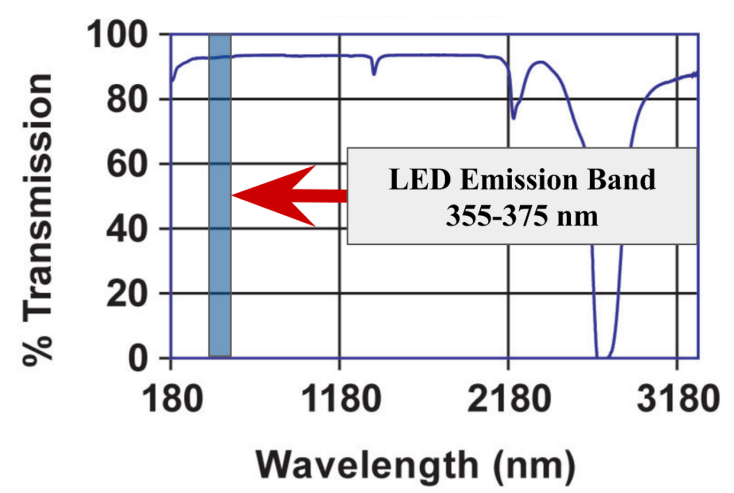

b) Absorbance by Photocatalyst Film

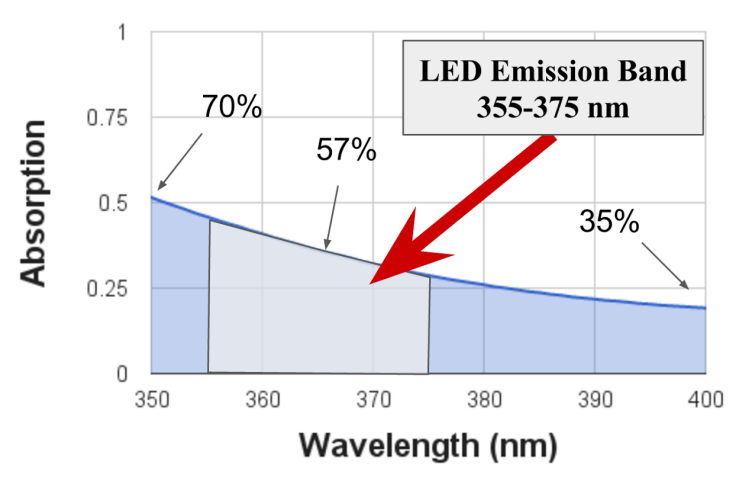

Figure 34: a) optical transmission spectrum for the fused silica support material (for a slide of $1 \mathrm{~cm}$ thickness) including an overlay to represent the wavelengths emitted by LEDs used for the illumination system; b) optical absorbance spectrum collected from the sol-gel derived film (20 wt\%), again including an overlay to indicate the LED emission band as well the \% transmission values for three locations on the curve.

To construct the illumination system, the LEDs were arranged into 6 LED strips connected in parallel, each alternating between having 7 LEDs and 8 LEDs in series. This alternation was done to stagger the LED positions on adjacent strips to both increase the uniformity of the light distribution within the reaction chamber and to provide necessary space for the LED mounting hardware and electrical connections. Photos of the illumination chamber are shown in Figure 35. 

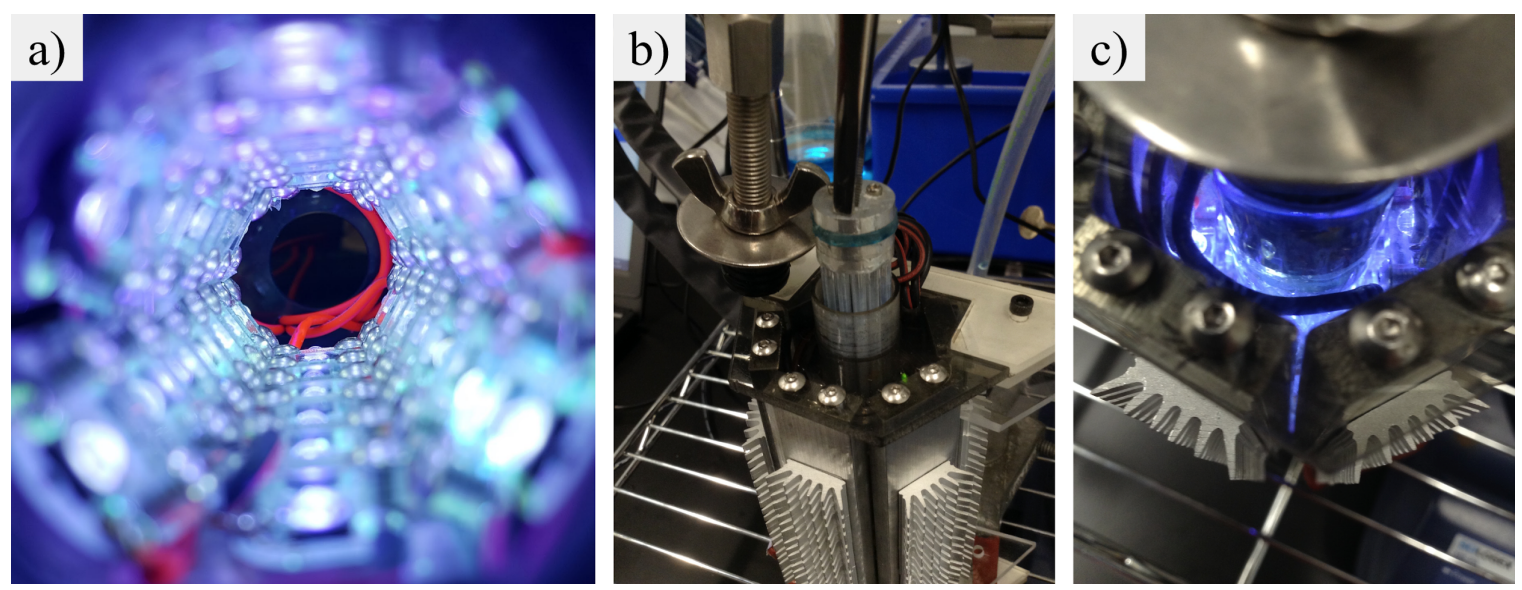

Figure 35: a) photo taken of the inside of the illumination system with the reaction chamber removed; b) photo showing the insertion of catalyst cartridge into the reaction chamber; c) image taken of the inside of the photoreactor while in operation.

The LEDs were powered by a Delta LED driver (LNE-36V), with the output intensity was controlled by an Arduino microcontroller via pulse width modulation (PWM). This functionality provides fine control of the radiative output power of the illumination system from $5 \%$ to $100 \%$, which was used to optimize the system settings for ideal reaction efficiency (these results are covered in Chapter 5).

\subsubsection{Auxiliary Systems}

The photoreactor is able to operate in two separate modes: 1) recirculation, and 2) continuous flow. In both cases the water is pumped from a reservoir - a $150 \mathrm{~mL} 3$-neck round-bottom flask - through the photoreactor. The difference between the modes is simply whether or not the reactor effluent is pumped back into the original reservoir or into an effluent reservoir. These two modes provide different sets of information, which will be explored in Chapter 5 . 

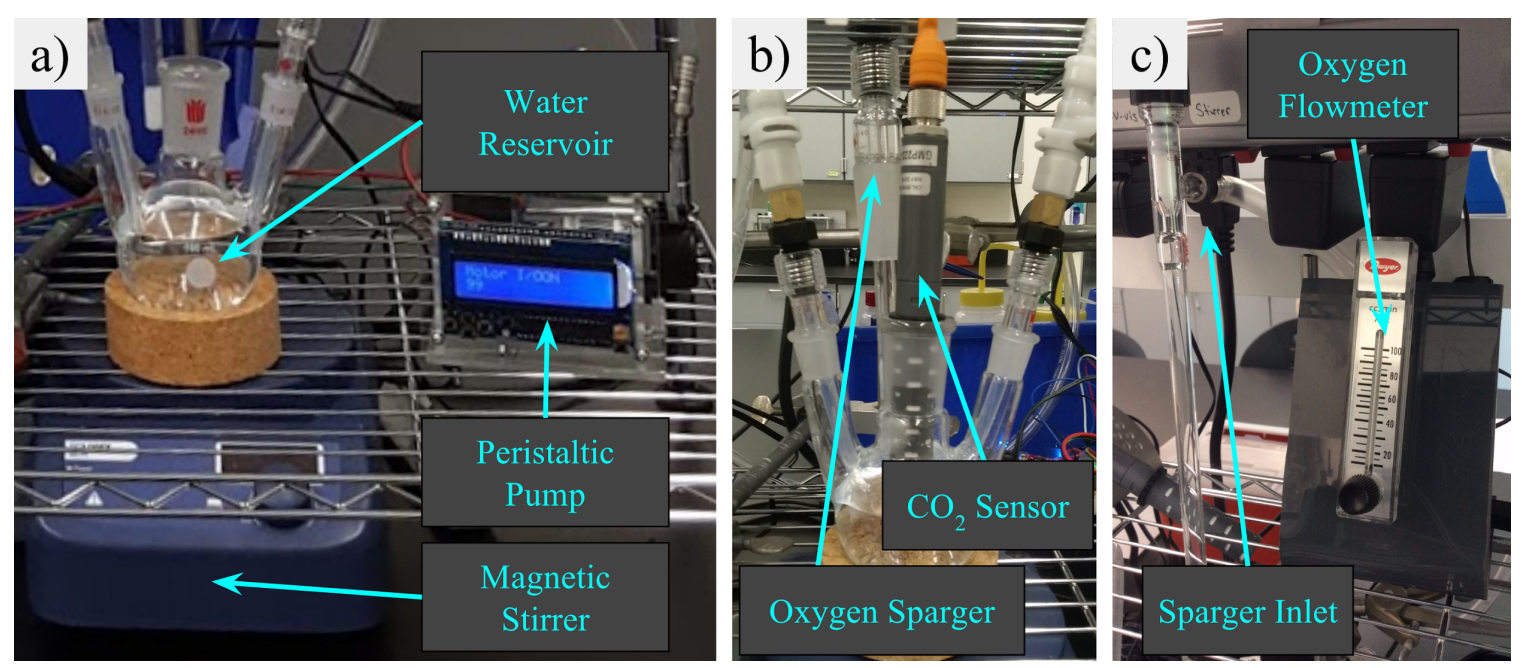

Figure 36: a) image of the water reservoir, mixing apparatus, and hydraulic pump; b) image of reservoir attachments - the water inlet and outlet utilize the 2 side necks and either a $\mathrm{CO}_{2}$ sensor or oxygen sparger is used in central neck depending on whether the experiment calls for recirculation or continuous flow mode; c) the oxygen sparger inlet and flowmeter.

The water circulation was provided by a peristaltic pump, controlled by another Arduino microcontroller with a push-button interface to control the pumping speed and status (Figure 36). Tygon tubing was used because its chemical stability to ensure that none of the organic markers or reaction intermediates react with the tubing walls. The tubing was connected to the 3-neck flask using a ground glass joint and quick-connect adapter. A PTFE coated stir bar was rotated at $300 \mathrm{rpm}$, by a magnetic stirrer below the flask, to maintain a uniform concentration marker in the water reservoir.

Because oxygen is consumed by the photocatalytic reaction, an oxygen sparger was also used to increase the dissolved oxygen concentration in the water. The oxygen flow rate was regulated and measured using a needle valve flowmeter with a range of $0-100 \mathrm{sccm}$. The oxygen was introduced into the water reservoir using a $400 \mathrm{~mm}$ sparger tube from Ace Glass that connects to the center neck of the flask. 


\subsubsection{The Analytical System}

\subsubsection{UV Flowcell Spectrometer}

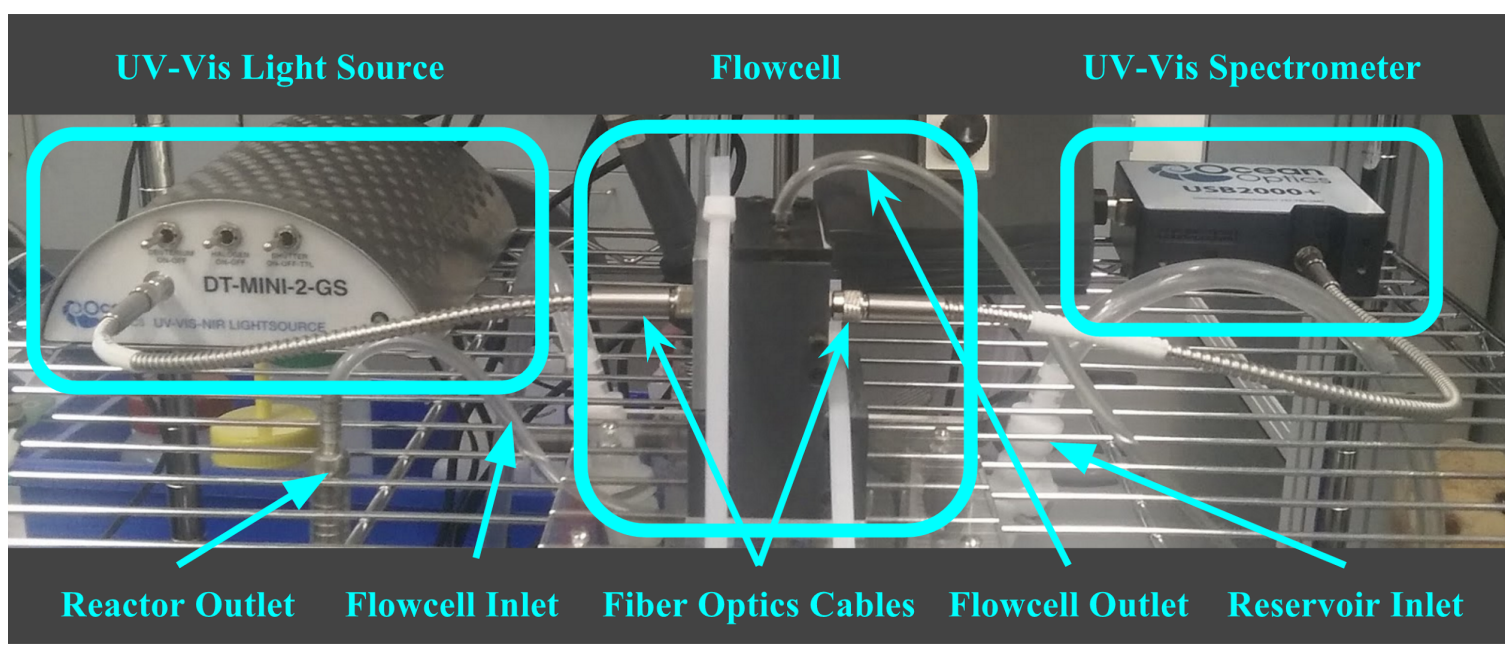

Figure 37: The UVFS system consists of the following: a flowcell cuvette - through which the reactor effluent passes; a UV-Vis light source that is channeled by fiber optical cable to the housing of the cuvette; and a UV-Vis spectrometer attached to the opposite side of the cuvette housing by a second fiber optic cable, allowing for measurement of effluent absorbance spectra.

The primary method chosen for analyzing the system was a custom designed concentration monitor that will be referred to as the UV-Vis Flowcell Spectroscope (UVFS). This method is relatively inexpensive and provides instant feedback of the system degradation performance. The UV-Vis light source was the DT-MINI-2-GS from Ocean Optics (deuterium and tungsten source), and the spectrometer used was the USB2000+, also from Ocean Optics. The setup is described in Figure 37.

Using the UVFS, the process shown in Figure 38 was implemented in order to calculate the EEO of this system. This involved the following stages: 1) water that has been contaminated with an organic marker is pumped from the water reservoir into the reaction chamber; 2) the ensuing photocatalytic reaction partially degrades the organic marker; 3) the water then exits the reaction chamber from the outlet and passes through 
the UVFS where the concentration of organic marker is detected; and 4) the water is collected in an effluent reservoir.
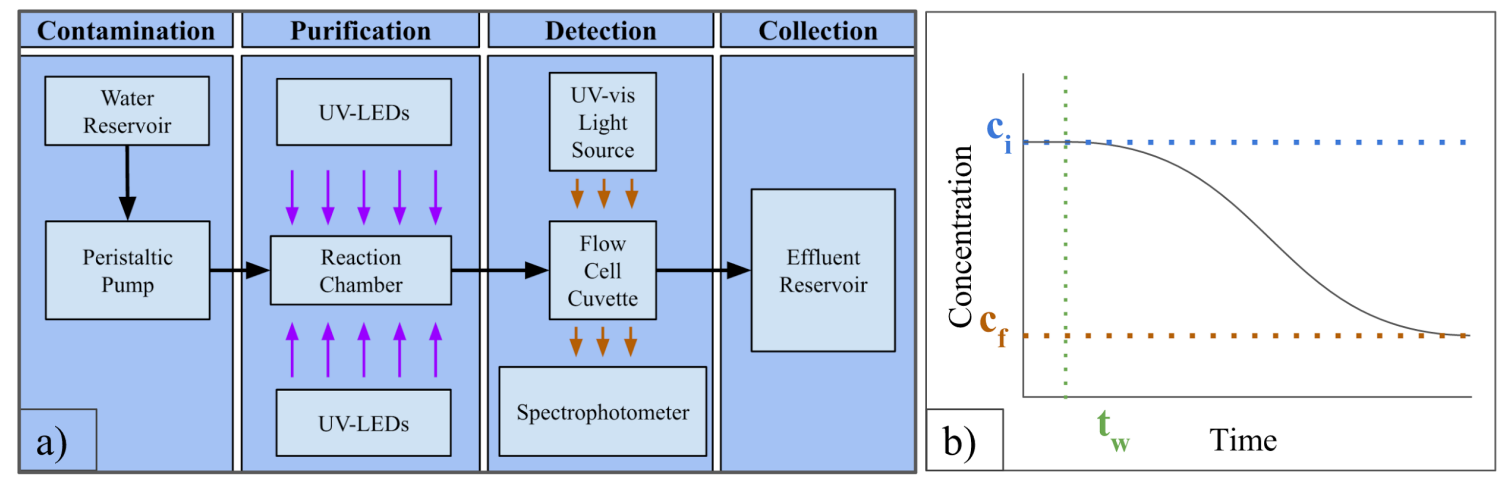

Figure 38: a) a schematic representation of the process used to evaluate system EEO; $b$ ) the theoretically expected result of a Switch-On event, allowing calculation of ci and $c f$ for use in Equation (16).

Before data collection, water from the reservoir is pumped through the system with the illumination system turned off. This initial period is to show that the conditions within the reaction chamber are in a steady state, confirming that the rate of adsorption and desorption of $\mathrm{MB}$ at the catalyst surface are balanced. Once the concentration has stabilized, data collection begins. After recording data for a wait time of 10 seconds $\left(t_{w}\right)$, the illumination system is switched on, resulting in a generalized sigmoid curve (Equation (39)) as the concentration $(C)$ changes from its initial value $\left(c_{i}\right)$ to a new steady state value $\left(c_{f}\right)$.

$$
C=c_{f}+\frac{\left(c_{i}-c_{f}\right)}{\left(1-e^{\sigma\left(t+t_{w}\right)}\right)}
$$

From these values, the system EEO can now be calculated using Equation (16), as described in from Chapter 1 . The constant, $\sigma$, can also provide information about the average residence time for molecules in the reactor. 


\subsubsection{Choice of Contaminants for Degradation Analysis}



Figure 39: Chemical structure and relevant properties for the two chosen contaminants, methylene blue and 2-propanol, along with the product of 2-PrOH oxidation, acetone.

Analysis by this method requires careful choice of contaminants, as there are many optical and chemical processes that must be considered. This research was carried out using 2 contaminants Methylene Blue (MB) and 2-Propanol (2-PrOH) because a) both degradation pathways are well understood, and b) they provide complementary information about the reaction process. The properties of each chosen contaminant are shown in Figure 39. MB allows for the reaction efficiency to be measured very accurately using the UVFS; and 2-PrOH provides confirmation that the degradation is occurring as a result of photocatalysis. This point is developed further through discussion of the contaminants themselves.

Methylene Blue is a standard organic marker used in many fields because of its strong and characteristic absorbance spectrum. This makes it an excellent contaminant to monitor by UVFS because its concentration can be measured very accurately. Additionally, it is nearly transparent in the $350-390 \mathrm{~nm}$ radiation given off by the 
illumination system. Ensuring that photolysis is minimized, thus confirming that the degradation is caused by photocatalysis and not direct reaction with the UV photons.

It is important to point out that because MB is a complex molecule, it breaks down into a vast variety of contaminants that can't be monitored with UVFS; this means that the degradation rate of TOC can only be equated to the degradation rate of $\mathrm{MB}$ when the concentration of $\mathrm{MB}$ is much greater than that of the sum of concentrations for each reaction intermediate [65]. Thus, the most accurate data for characterizing the degradation occurs before the reaction proceeds long enough to create a significant amount of reaction products.

There has been some controversy regarding the use of dyes for the evaluation of photocatalytic degradation. This is due to the fact that the results can be misleading if experiments are not done carefully $[19,66]$. The problem occurs when the dye itself absorbs the UV light and transfers an excited electron to the semiconductor material as is exploited in dye-sensitized solar cells. This can cause degradation of the dye, but the mechanism of the degradation is photocatalysis.

However, this issue is easily avoidable as long as one chooses contaminants that have very low absorbance in the wavelength range that is emitted from the illumination system, which is true for the LED emission chosen for the reactor and MB. Nevertheless, it is prudent to confirm that photocatalytic oxidation is occurring in the reactor.

This was done with the second contaminant: 2-PrOH. Since 2-PrOH is completely transparent in the UV and visible spectrums, it is not detectable by UVFS and is not at risk for absorption of photons emitted by the UV-LEDs. However, when oxidized 2- 
$\mathrm{PrOH}$ is converted to acetone, which does have absorbance peaks in the UV (195 and 277 $\mathrm{nm})$. This means that it is possible to detect the formation of the reaction product rather than the degradation of the initial contaminant. Furthermore, experimental findings indicate that oxidation of 2-PrOH results in an inversely proportional relationship between the concentration of 2-PrOH and acetone in the early stages of the reaction [48, $52,67]$, indicating that the TOC degradation rate for the reaction can be deduced by monitoring the formation rate of acetone in the solution.

\subsubsection{UVFS Calibration}

Before the system EEO can be calculated from Equation (39) it is necessary to correlate the absorbance values detected by the UVFS with the concentration of MB. This was done without a catalyst cartridge in the reaction chamber to prevent a change in concentration due to absorbance of MB onto the catalyst surface. The following procedure was used:

1. Begin pumping pure DI water through system (no MB added)

2. Collect a dark reference spectrum (light source off) to establish baseline

3. Collect a light reference spectrum (light source on)

4. Zero the sensor by subtracting the light reference spectrum from the live data

5. Add MB stock solution of known concentration to water reservoir

6. Circulate water until the concentration detected by UVFS stabilizes

7. Collect absorbance data for 2 seconds and average result

8. Repeat steps 5-7 for until 8 data points have been collected

The results are shown are shown in Figure 40. The greatest accuracy of the spectrometer, as declared by the manufacturer, is between absorbance values of 0.5 and 1.0. Therefore, the concentration range chosen for EEO determination experiments was $\sim 2-3 \mathrm{ppm}$. 


\section{Sensor Calibration Chart}

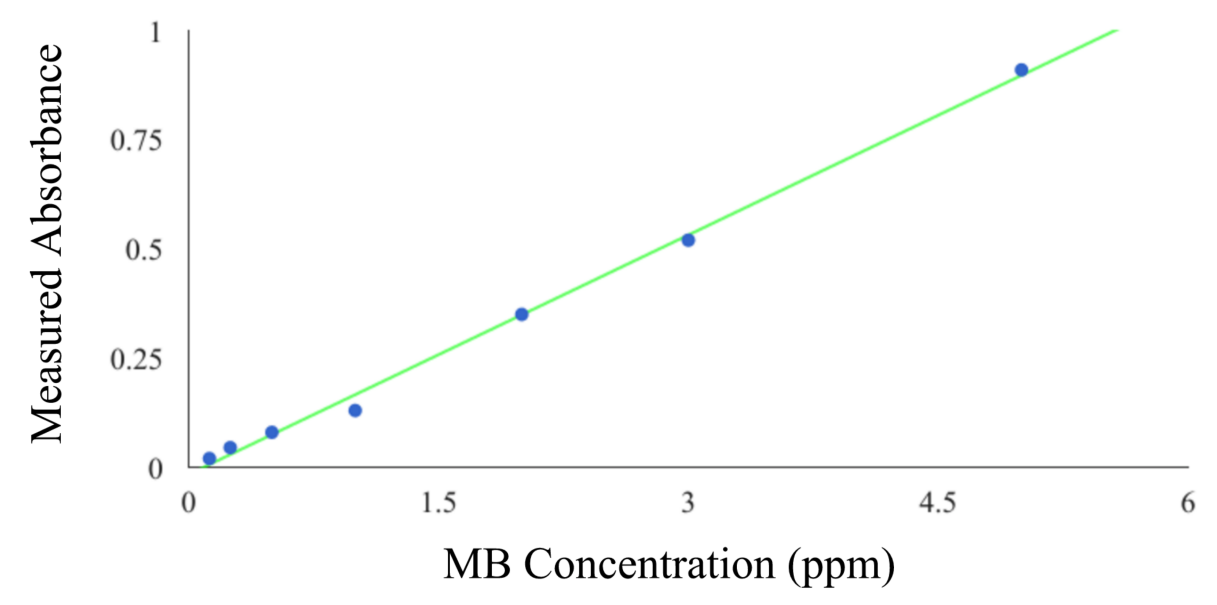

Figure 40: UVFS calibration data used to map absorbance values to MB concentration.

\subsubsection{4 $\mathrm{CO}_{2}$ Sensor}

The device used for detecting $\mathrm{CO}_{2}$ emission was a gaseous $\mathrm{CO}_{2}$ sensor (GMM222 from Vaisala), calibrated for use in a range from 0-2000 ppm. Data from this sensor was collected by an Arduino microcontroller for analysis. This sensor uses an infrared emitter, reflector, and detector, all encased within a $40 \mathrm{~mm}$ probe tip. The tip of the sensor was inserted into the headspace of the 3 neck flask and sealed with 3 silicone rubber o-rings (shown in Figure 36). Since the headspace of the flask was sealed, measurements from the sensor represent a cumulative sum of $\mathrm{CO}_{2}$ given exhausted during the entire reaction. Also, because the flask needed to remain sealed, these measurements were only collected in recirculation mode (as opposed to continuous-flow mode) to prevent pressure build-up in the effluent reservoir. 


\section{Chapter 5: Results}

The quantitative results obtained from the silica slide experiments and from the photoreactor degradation experiments are presented in this chapter. A discussion section is also included to compare the results with other photocatalytic reactors and to relate the results from the slide experiments to the EEO results from the photoreactor.

\subsection{Photocatalyst Optimization}

\subsubsection{Thickness Analysis by Optical and Scanning Electron Microscopy}

In the previous chapter, it was noted that experiments were carried out to determine the practical range of film thicknesses that were derivable from the SG dip coating method. The film thickness was controlled by adjusting the viscosity of the dipping solution, which was determined to be practical for solutions containing an SG concentration from 5-30 wt\% (i.e. an EtOH concentration of 70-95 wt\%). In order relate these results to the theoretical hypothesis from Chapter 2 , it is necessary to quantify the effect of SG wt\% on the thickness of the resulting film.

To do this, a technique was developed for determining a thin-film thickness with a simple and non-destructive method using optical spectroscopy. To begin, a calibration chart was created to correlates optical absorbance with thickness measurements via Scanning Electron Microscopy (SEM). During this process, sacrificial samples were created and analyzed by SEM, but once the correlation was made, future samples were able to be analyzed quickly, cheaply, and non-destructively. 
The thickness of the coating was adjusted by controlling the SG wt $\%$ through serial dilution with absolute ethanol, while the dip coating and heat treatment parameters were held constant. Symbolizing, the weight percent of SG in the original solution as W, then the serial dilutions resulted in weight percents of $0.5 \mathrm{~W}, 0.4 \mathrm{~W}, 0.3 \mathrm{~W}$, and $0.2 \mathrm{~W}$; these are labeled in the figures as S1-S4 respectively, an uncoated quartz substrate was used as a reference and is labeled as S0. An example of the SEM analysis of one sample (S4) is shown below in Figure 41.

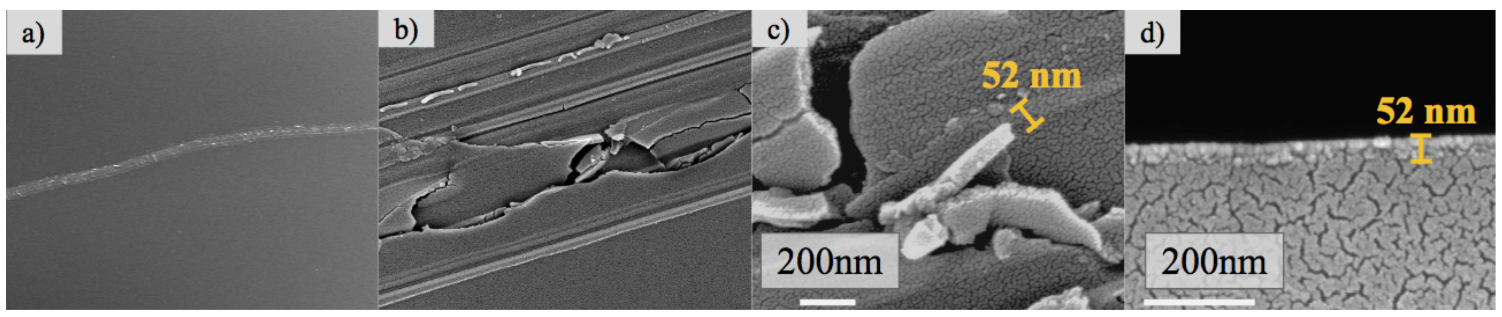

Figure 41: SEM images of the coating. a) and b) A scratch was made along the entire length of the coating using a diamond scribe in order analyze the coating thickness, and to verify the thickness consistency. c) Broken pieces of the coating oriented perpendicular to the detector were used for measurements. d) A cross section of the sample was created to verify coating thickness.

The optical transmittance of each sample was then measured by a Shimadzu UV-3600 spectrophotometer in the range of 250-500 nm. The results are shown in Figure 42 along with the calculated absorbance spectra. It was hypothesized that the films would have a thickness that is directly proportional to the titanium weight percent, and further, that the peak $(257 \mathrm{~nm})$ optical absorbance should be directly proportional to the film thickness. This is justified theoretically by the fact that the optical transmittance of the film exponentially decays with increasing thickness as established by the Beer-Lambert Law. Since absorbance is calculated by taking the negative logarithm of transmittance, the result should be proportional to the film thickness. 

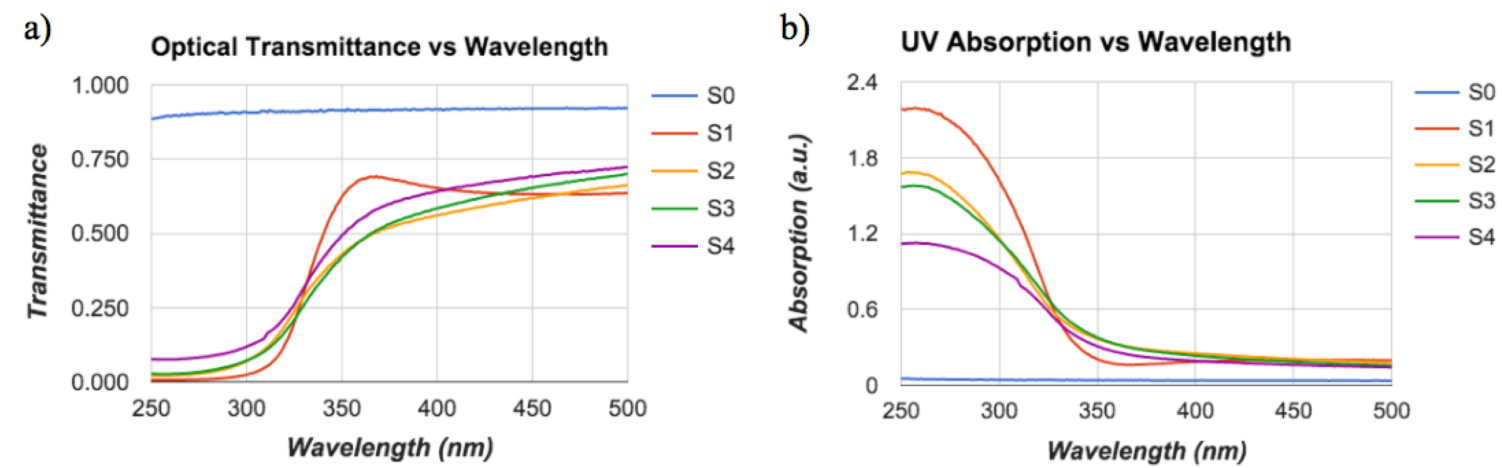

Figure 42: UV-vis spectra from 250-500 $\mathrm{nm}$ showing a) the optical transmittance, and b) the optical absorbance, for samples SO-S4.

In order to test the hypothesis, the relative absorbance was plotted against the relative weight percent for each sample and is shown in Figure 43 along with a dotted line depicting the theoretical prediction for proportionality $(y=x)$. The correlation coefficient for the data is 0.97 , confirming that the coating thickness is, in fact, proportional to Ti weight percent.
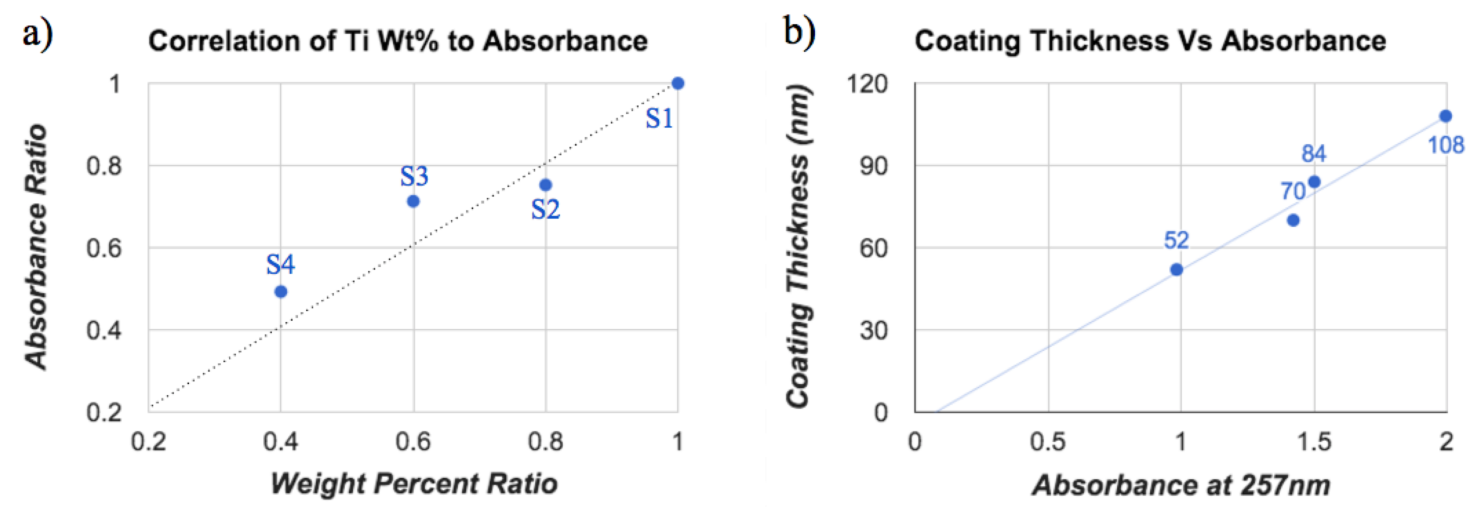

Figure 43: a) Correlation data used to verify the proportionality of Ti weight percent in sol-gel to peak absorbance values of the resulting films. b) Coating thickness data plotted against peak absorbance values to obtain mathematical relationship ( $t=56.2 a$ -4.4 ).

The equation relating the absorbance measurements to the film thickness was then determined by plotting each thickness measurement $(\mathrm{t})$ from the SEM analysis vs. its respective absorbance value (a), and finding a linear fit to the data. The correlation coefficient for this data was also calculated, and has a value of 0.99 . The fact that the 
correlation between absorbance and thickness is higher than that between weight percent and thickness indicates that most of the deviation from theory was introduced in the coating process, rather than in the measurement. The resulting line is expressed mathematically as $\mathrm{t}=56.2 \mathrm{a}-4.4$ and is plotted in Figure $3 \mathrm{~b}$; this equation permits simple and non-destructive determination of the coating thickness via optical transmission measurement.

\subsubsection{Activity Optimization}

In Chapter 4, experiments involving the implantation of both $\mathrm{TiO}_{2}$ and $\mathrm{SiO}_{2}$ nanoparticles into the dipping solution were described. The preliminary results indicated that up to $0.3 \mathrm{wt} \%$ of $\mathrm{TiO}_{2}$ particulate, and $0.05 \mathrm{wt} \%$ of $\mathrm{SiO}_{2}$ particulate, could be added into the dipping solution before adverse effects on film uniformity became noticeable. The motivation for this modification was to increase surface roughness and thus the catalytic surface area of the resulting films, as well as to reduce electron-hole recombination rate through the introduction of hole trapping sites throughout the film. In order to quantify the effect of these modifications on system effectiveness, it is necessary to compare the photocatalytic ability of each film.

The thickness and concentration of implanted nanoparticles were varied, while the ability of each film to degrade an organic marker that has been adsorbed to the film surface was measured by UV-vis spectroscopy. In order to remain consistent with the photoreactor degradation experiments, MB was used as the organic marker.

A small apparatus was built to allow for quantification of UV attenuation, as well as the photodegradation rate of a film. The apparatus is shown in Figure 44, and consists of a 
UV-LED (the same type as used for the illumination system of the photoreactor), a slide holder, and a photomask. The entire apparatus is contained in a black box to prevent ambient light from contributing to the reaction. To prepare, a $\mathrm{TiO}_{2}$ coated slide was spincoated with an aqueous solution $(10 \mathrm{mg} / \mathrm{L})$ of $\mathrm{MB}$, and then dried. Reference UV-vis spectra were acquired before and after the MB spin-coating. Next, the slide was placed into the reaction cuvette that contained $300 \mu \mathrm{L}$ of water, and a photomask was placed on the outside of the cuvette in order leave a $1 \mathrm{~cm}^{2}$ region unmasked.
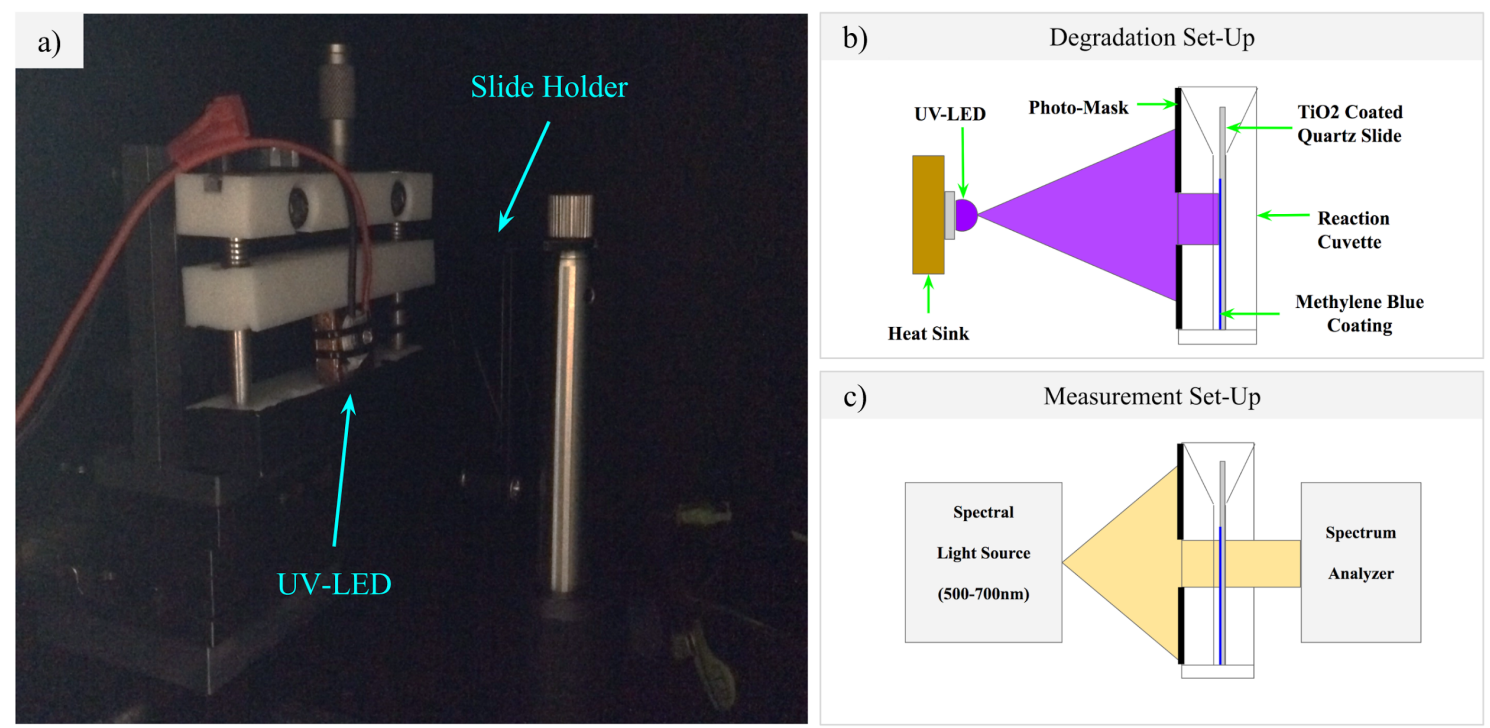

Figure 44: a) experimental set-up for controlled exposure of the MB coated slides; $b$ ) schematic diagram describing the exposure process; c) schematic set-up describing the measurement process by UV-vis spectroscopy.

A series of illumination and measurement events were then carried out. First, the LED was switched on for 15 seconds and then turned off again. The amount of MB remaining on the surface is determined by acquiring another UV-vis absorption spectrum. This is done by removing the cuvette from the illumination apparatus, and placing directly into the cuvette holder of a spectrophotometer. Acquisition wavelengths of the spectrometer were restricted to the range of $500-700 \mathrm{~nm}$, so that the no degradation of $\mathrm{MB}$ was caused 
by the measurement itself. Once the 15 second spectrum was collected, the cuvette was again placed in the illumination chamber and illuminated for another 15 seconds, followed by another measurement in the UV-vis. This process was then repeated until the MB peak in UV-vis spectrum could no longer be reduced - typically after 1-2 minutes of illumination.

a) MB Degradation on Quartz Slide
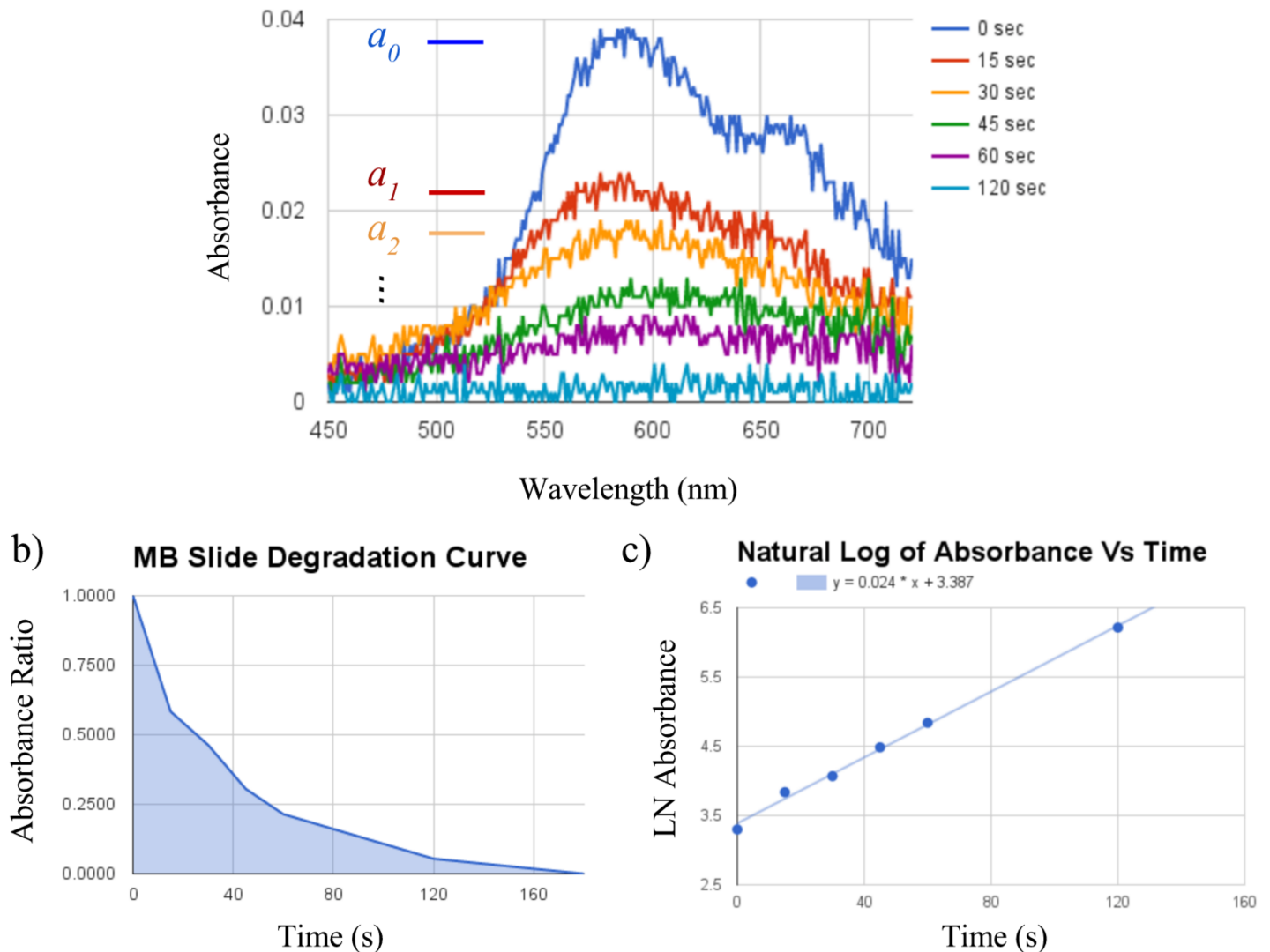

Figure 45: a) raw data from activity measurement of the $5 \mathrm{wt} \%$ control sample; b) plot of absorbance peak heights (a0-a5) vs time; c) plot of the negative natural log of absorbance values vs time and the resulting linear trend line.

The MB peak absorbance value for each interval $\left(a_{i}\right)$ is divided by the peak value at 0 seconds $\left(a_{0}\right)$, yielding the relative amount of $\mathrm{MB}$ on the slide as a function of time. This allowed each sample to be characterized by its decay constant, which was determined by 
plotting the negative natural log of absorbance versus duration of illumination and finding the slope of the resulting trendline. Note that because there is overlap of the 580 $\mathrm{nm}$ and $668 \mathrm{~nm}$ absorption peaks, each absorption spectra were fit with two gaussian peaks and deconvolved using a software called Fityk (0.9.8). The result of this process, conducted with a sample made from a $5 \mathrm{wt} \%$ solution is shown in Figure 45.

Although this method produced good results on an individual basis, comparison of different sample was difficult due to a high variance when attempting to repeat degradation results $(\sim 15 \%)$. It was discovered that issue was being caused by the water inside the cuvette, whichcaused desorption of the $\mathrm{MB}$ from the $\mathrm{TiO}_{2}$ in a non reproducible way when the slide was inserted. Therefore, the same procedure was carried out without water. This change caused reproducibility to improve to less than $5 \%$ variance. Additionally, the reaction order changed from a $1^{\text {st }}$ order reaction to a $0^{\text {th }}$ order reaction because all $\mathrm{MB}$ remained on the catalyst surface, eliminating mass transfer limitation.

With this change implemented, the effect of thickness on photoactivity was observed. Four samples were created with increasing sol-gel concentration within the range of practical viscosity (5-20 wt $\%)$, which are labeled as T-1 through T-4. Then, to observe the effect of nanoparticle implantation, 7 samples were created: the first was a control sample made from a $5 \mathrm{wt} \%$ solution with no nanoparticulate added; followed by three with increasing concentration of $\mathrm{TiO}_{2}$ added to a $5 \mathrm{wt} \%$ solution; two with $\mathrm{SiO}_{2}$ added; and finally one with both $\mathrm{TiO}_{2}$ and $\mathrm{SiO}_{2}$ added. These are labeled I-1 through I-6. The name given to each sample, the solution composition, and the relative photoactivity of the 
resulting film is presented in Table 4. This table also lists normalized rate constant determined from each sample.

\begin{tabular}{|c|c|c|c|}
\hline \multirow{3}{*}{ Experiment } & $\begin{array}{c}\text { Sample } \\
\text { ID }\end{array}$ & Description of Fabrication Process & $\begin{array}{c}\text { Normalized } \\
\text { Rate } \\
\text { Constant }\end{array}$ \\
\hline \multirow{3}{*}{ Thickness } & $\mathrm{T}-1$ & $5 \mathrm{wt} \%$ Ti sol-gel & 1.01 \\
\cline { 2 - 4 } & $\mathrm{T}-2$ & $10 \mathrm{wt} \% \mathrm{Ti}$ sol-gel & 1.36 \\
\cline { 2 - 4 } & $\mathrm{T}-3$ & $15 \mathrm{wt} \% \mathrm{Ti}$ sol-gel & 1.93 \\
\hline \multirow{3}{*}{ Implantation } & $\mathrm{T}-4$ & $20 \mathrm{wt} \% \mathrm{Ti}$ sol-gel & 0.99 \\
\hline & $\mathrm{I}-1$ & $5 \mathrm{wt} \% \mathrm{Ti}$ sol-gel & 1.12 \\
\hline & $\mathrm{I}-2$ & $5 \mathrm{wt} \% \mathrm{Ti}$ sol-gel $+0.1 \% \mathrm{TiO}_{2}$ & 1.75 \\
\hline & $\mathrm{I}-3$ & $5 \mathrm{wt} \% \mathrm{Ti}$ sol-gel $+0.2 \% \mathrm{TiO}_{2}$ & 2.24 \\
\hline & $\mathrm{I}-4$ & $5 \mathrm{wt} \% \mathrm{Ti}$ sol-gel $+0.3 \% \mathrm{TiO}_{2}$ & 3.10 \\
\hline & $\mathrm{I}-5$ & $5 \mathrm{wt} \% \mathrm{Ti}$ sol-gel $+0.05 \% \mathrm{SiO}_{2}$ & 2.99 \\
\hline Optimized & $\mathrm{I}-6$ & $5 \mathrm{wt} \% \mathrm{Ti}$ sol-gel $+0.10 \% \mathrm{SiO}_{2}$ & 5.23 \\
\hline
\end{tabular}

Table 4: Summary of synthesis conditions and the normalized rate constant for each sample. Samples T-1 and I-1 were prepared from identical synthesis conditions.

Raw data for the $5 \mathrm{wt} \%$ control sample (I-1) along with its corresponding degradation curve and the analyzed results for each solution variation are shown in Figure 46. The normalized rate constant was finally calculated by dividing each rate constant by the averaged degradation rate from samples T-1 and I-1, which were prepared with an identical synthesis procedure.

The results from the thickness experiment show that the film thickness was approximately proportional to degradation rate. This is due to the fact that the an increased thickness will absorb a greater fraction of incident UV photons. However, this behavior is expected only in the thin-film regime since the fraction of escaped (transmitted) photons asymptotically approaches zero as the film thickness increases. Therefore, increasing the film thickness has a diminishing return. However, as reported in Section 4.1.3, films using dipping solution greater than $20 \mathrm{wt} \%$ were determined 
impractical due to high density of crack formation. Therefore, it was concluded that the concentration for cartridge fabrication was $20 \mathrm{wt} \%$, which resulted in a $\mathrm{TiO}_{2}$ film of 230 nm thickness.
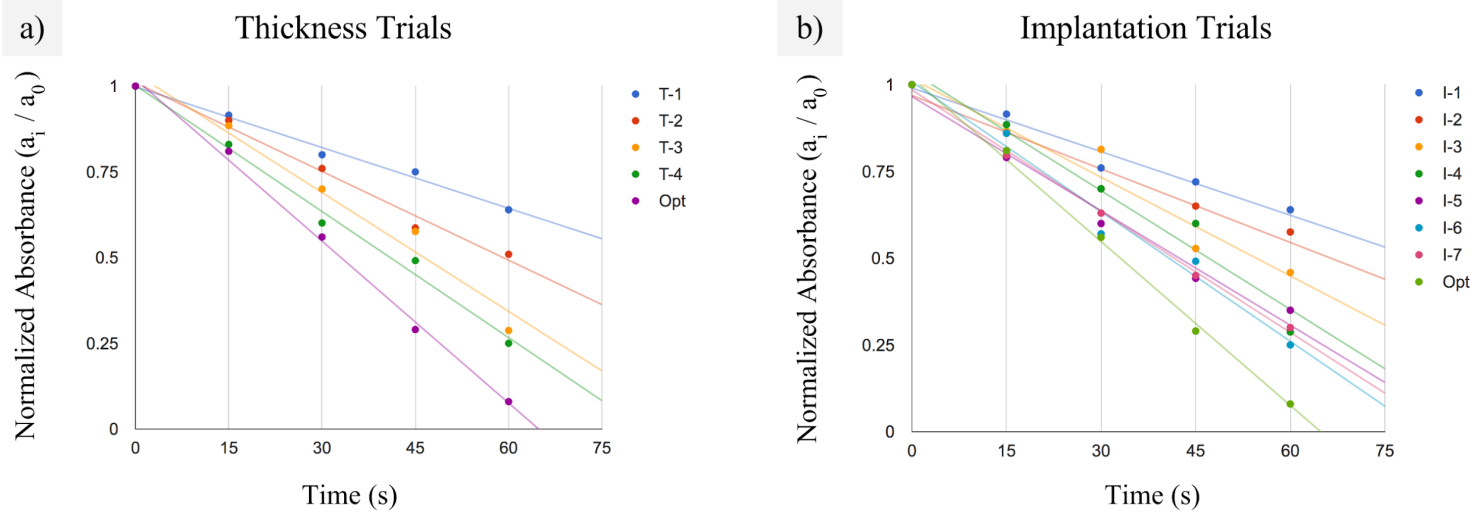

Figure 46: a) Degradation rate comparison for films with increasing thickness; b) Degradation rate comparison for films modified by nanoparticle implantation. (Sample descriptions provided in Table 4.

From the second experiment, one can see that modifications of the $\mathrm{TiO}_{2}$ base film (I-1) had a significant impact on the measured degradation rate. As hypothesized, the addition of $\mathrm{TiO}_{2}$ particles into the solution did increase the degradation rate. This was attributed to the increased catalyst surface area as well as increased rutile content. However, a much greater effect was seen through the addition of $\mathrm{SiO}_{2}$ particles into the solution. Interestingly, this effect slightly decreased when the $\mathrm{SiO}_{2}$ content was double from 0.05 $\mathrm{wt} \%$ to $0.10 \mathrm{wt} \%$. Inspection of the film by optical microscope revealed that the addition $0.10 \mathrm{wt} \% \mathrm{SiO}_{2}$ caused non-uniformity of the film due to agglomeration of particles.

Based on these results, an optimized sample was created from a solution of $20 \mathrm{wt} \%$ Ti solgel, $0.3 \% \mathrm{TiO}_{2}$ and $0.05 \% \mathrm{SiO}_{2}$. Analysis of this film determined that the optimized 
combination of modifications led to an increase in degradation rate of $523 \%$, relative the two control samples.

\subsection{Photoreactor Efficiency}

Using the method of analysis described in Section 4.2.2.1, the effect of various system modifications on the EEO value was determined. The first objective was to utilize and verify the results of the activity optimization results presented in Section 5.1.2. The second objective was to determine the optimal illumination intensity for this particular system, in order to establish the standard operating condition.

\subsubsection{Prerequisite Measurements}

\subsubsection{First-Order Reaction Rate Confirmation}

To verify that Equation (16) is valid for calculating the system EEO, it was necessary to verify that 1 st order reaction kinetics were observable for this system. This was done by measuring the concentration of $\mathrm{MB}$ as a function of time while the water is recirculated through the reactor with the illumination system on. A standard order verification test was performed by plotting the absorbance, natural log of absorbance, and inverse of absorbance each vs. time to check which is the most linear. As can be seen in Figure 47, an approximately linear trend is observed for the 1 st order test. Whereas the $0^{\text {th }}$ order and $2^{\text {nd }}$ order tests were clearly non-linear. 
a)

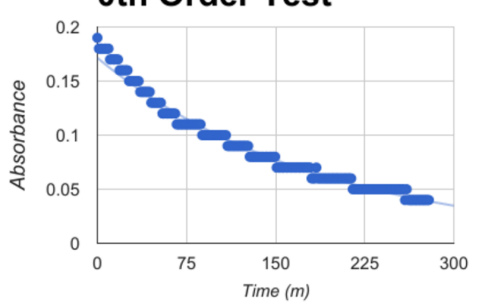

b)

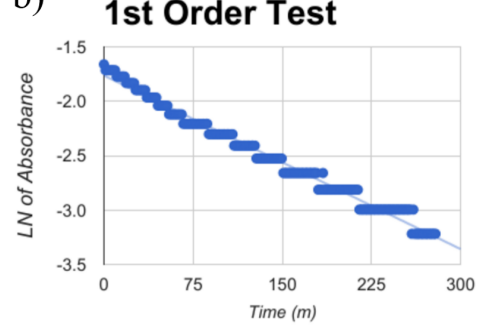

c) 2nd Order Test

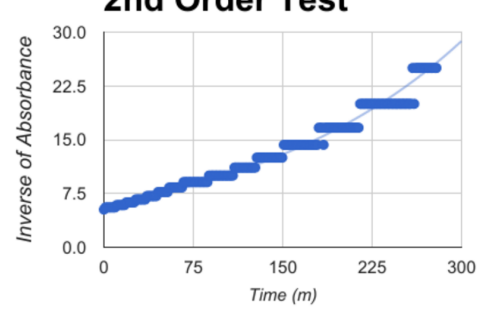

Figure 47: a) plot of absorbance vs. time; b) plot of the natural log of absorbance vs. time; c) plot of the inverse of absorbance vs. time. The fact that the natural log of absorbance is approximately linear verifies that the reaction has first order kinetics.

\subsubsection{Oxidation Confirmation}

As described in Section 4.2.2.2, it is prudent to confirm oxidation of the contaminant molecules in order to verify that degradation of contaminant molecules is occurring due to due oxidation reactions. To test for oxidation confirmation, 2-PrOH was added to the water reservoir to obtain a concentration of $50 \mathrm{mg} / \mathrm{L}$. The water was then pumped through the reactor for 10 min with the illumination system off to ensure that adsorption and desorption processes were in equilibrium. Because 2-PrOH is completely transparent to UV and visible wavelengths, there was no change to the absorption spectrum from the water reference spectrum. The illumination was then turned on. As the reaction proceeded, an absorption peak slowly formed at $195 \mathrm{~nm}$, precisely where the acetone absorption peak was expected. Figure 48 shows the height of the $195 \mathrm{~nm}$ absorbance peak as a function of time. An approximately linear increase in peak height was observed - verifying that the 2-PrOH was successfully being converted to acetone through oxidation. 


\section{UV Absorbance at $195 \mathrm{~nm}$}

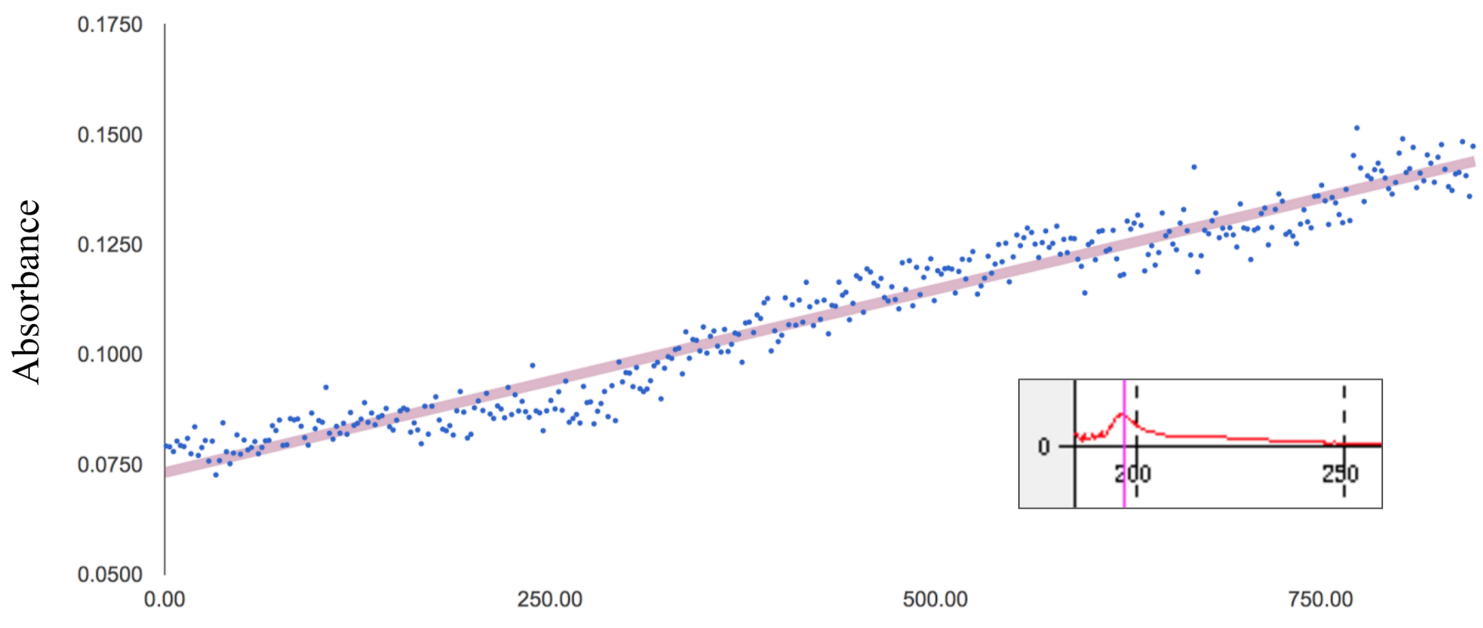

Time (s)

Figure 48: Plot of absorption peak value at $195 \mathrm{~nm}$ vs. time obtained from the UVFS system. An inset plot shows the UVFS absorbance spectrum obtained at 500 seconds.

\subsubsection{Characterization of Continuous Flow Degradation Behavior}

Finally, it is necessary to observe the result of the reactor when operated in continuousflow mode. This is the most desirable mode of operation for industrial processes and is the best way to characterize the system EEO, as described in Section 1.2.2 and Section 4.2.2.1. This means that rather than recirculating the water from the reservoir, the effluent is collected in a separate container to maintain a constant $\mathrm{MB}$ concentration for the water entering the reactor. The objectives of this experiment were as follows:

- Observe change in concentration of MB when reactor is switched on (Switch-On)

- Determine the average MB concentration once the effluent reaches a steady state

- Observe reversal of MB concentration when reactor is switched off (Switch-Off) 


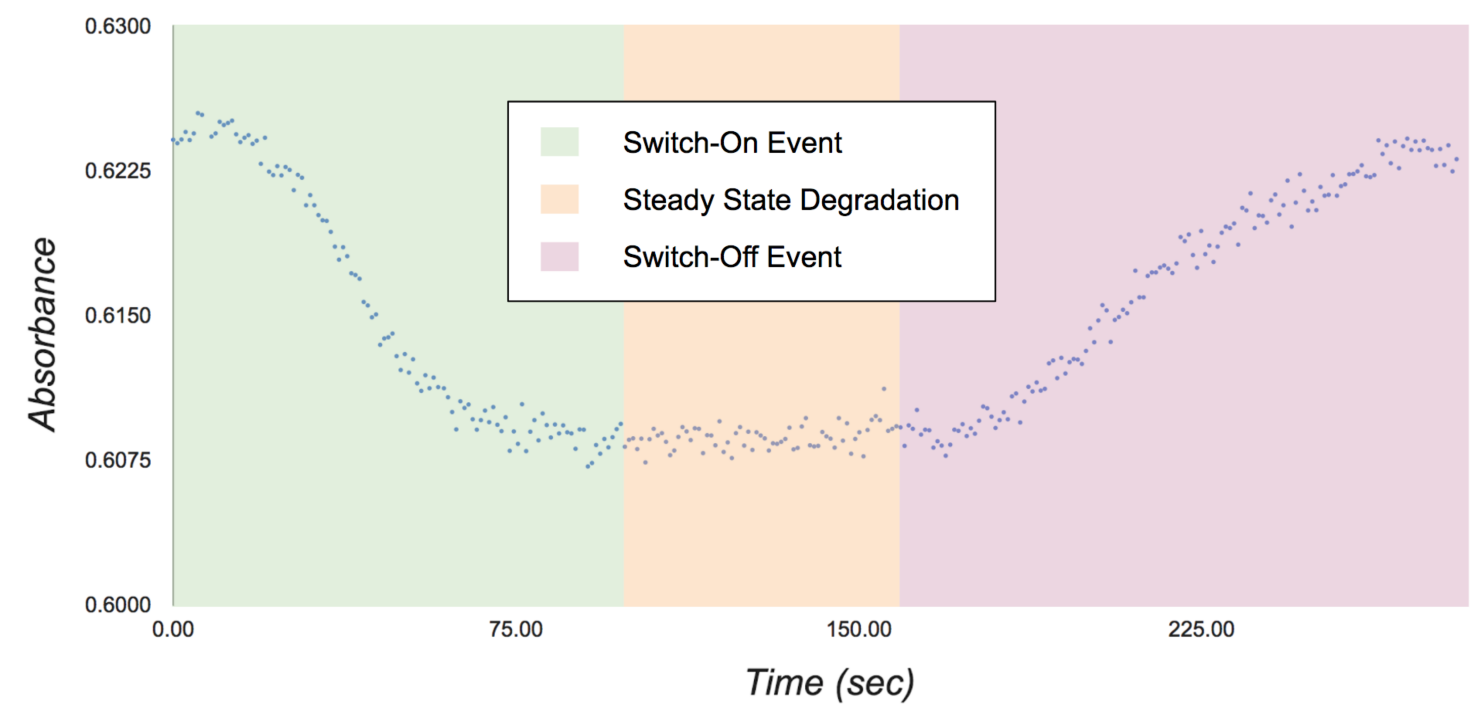

Figure 49: Data acquired from tracking the $580 \mathrm{~nm}$ MB absorption peak as the reactor is turned on and then turned off again. The concentration changes from its initial concentration to a steady state concentration when the illumination is switched on. When the illumination is switched off again, the concentration returns to the initial concentration.

Data collected from the experiment conducted using using a cartridge synthesized from the same dipping solution and procedure as used for the T4 sample described in Table 4 and an illumination intensity of $10 \%$. The experiment was also repeated 8 times to determine the coefficient of variation $\left(\mathrm{C}_{\mathrm{v}}\right)$ for concentration change measurements. Initially, the results showed a high $\mathrm{C}_{\mathrm{v}}$ of $8.2 \%$. However, the source of this uncertainty was determined to be the mechanism for switching the illumination system on and off, as there was a delay in the signal from the illumination system PWM microcontroller. Installing an analog toggle switch between the illumination power supply and photoreactor LEDs decreased the $\mathrm{C}_{\mathrm{v}}$ to $1.5 \%$.

Taking an average of the first 10 data points (approximately 10 seconds of data collection) provides the absorbance value for calculating the initial concentration, $c_{i}$. The last 10 data points of the Switch-On event region provide average absorbance value for 
calculating the final output concentration., $c_{f}$. These two values allow calculation of the EEO for this reaction from Equation (16). For the data from the Switch-On event shown in Figure $49, \log _{10}\left(c_{i} / c_{f}\right)$ has a value of $\log _{10}(0.625 / 0.608)=1.12 \times 10^{-2}$; the flow rate, $F$, was measured to be $0.0036 \mathrm{~m}^{3} / \mathrm{hr}(56 \mathrm{~mL} / \mathrm{min})$; and the power consumed by the illumination system, $P$, was $0.013 \mathrm{~kW}$, found from current and voltage drawn by the LEDs $\left(2.7 \times 10^{-3} \mathrm{~kW} \times 45 \times 10 \%\right)$ Therefore the EEO for this reaction is found from a simple computation:

$$
E E O=\frac{0.013(\mathrm{~kW})}{0.0036\left(\mathrm{~m}^{3} / \mathrm{h}\right) \times 1.12 * 10^{-2}}=299 \mathrm{kWh} / \mathrm{m}^{3}
$$

\subsubsection{System Optimization}

\subsubsection{Photocatalyst Film Composition}

Five cartridges were fabricated in order to determine the effect of film modification on the system EEO value. These cartridges were tested while all illumination and flow conditions were held constant. The first cartridge tested did not have a photocatalyst film at all, and was used as an experimental control; this cartridge was the 3D silica support material only, without having been dip coated or heat treated. The other 4 cartridges, AD, were fabricated based on the results of the film activity optimization experiments. The specific solution compositions were as follows: A) $5 \mathrm{wt} \% \mathrm{Ti}$ sol-gel, B) $5 \mathrm{wt} \% \mathrm{Ti}$ solgel $+0.3 \mathrm{wt} \% \mathrm{TiO}_{2} ;$ C) $20 \mathrm{wt} \% \mathrm{Ti}$ sol-gel $\left.+0.3 \mathrm{wt} \% \mathrm{TiO}_{2} ; \mathrm{D}\right) 20 \mathrm{wt} \% \mathrm{Ti}$ sol-gel $+0.3 \mathrm{st}$ $\% \mathrm{TiO}_{2}+0.05 \mathrm{st} \% \mathrm{SiO}_{2}$.

For each trial, the EEO and $\log _{10}\left(\mathrm{c}_{\mathrm{i}} / \mathrm{c}_{\mathrm{f}}\right)$ were calculated as described in the previous section. The absorption data from the Switch-On event obtained from cartridge A was 
converted to MB concentration, and is shown in Figure 50. Although the control cartridge did show a measurable decrease in the MB absorbance peak, it was negligible for practical purposes, showing only a $0.0005 \%$ reduction in absorbance, equivalent to an EEO value $>10,000 \mathrm{kWh} / \mathrm{m}^{3}$. In comparison, the EEO value for cartridge A was 299 $\mathrm{kWh} / \mathrm{m}^{3}-\mathrm{a} 97 \%$ reduction from the control. The results of the four non-control cartridges are also presented, which verify that significant enhancement of the system EEO can be obtained through photocatalyst film modification. Specifically, there was 81.9\% decrease in EEO between the $\mathrm{TiO}_{2}$ base cartridge, $\mathrm{A}$, and the optimized cartridge, D.
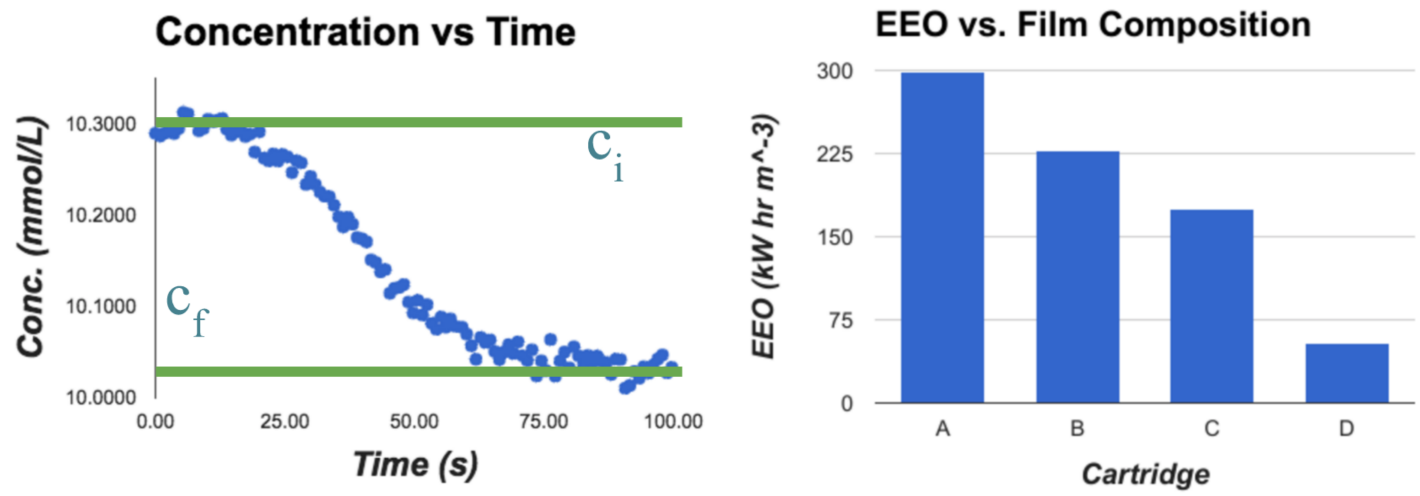

Figure 50: a) Concentration data acquired via FCUVS, showing the change in MB concentration during the transition from a System-Off state to System-On state; $b$ ) system EEO values determined using four different cartridges, $A-D$, and $10 \%$ intensity.

\subsubsection{Illumination Intensity}

In order to determine the effect of UV intensity on the EEO values, the intensity of UV output from the illumination system was varied and the EEO was measured for each trial. This was accomplished using PWM as described in Section 4.2.1.2 to vary the duty cycle from $10 \%$ to $60 \%$ in $10 \%$ increments, which was assumed to correspond directly to the 
percentage of power consumption. The results for each of the six trials are in plotted in

\section{Figure 51.}

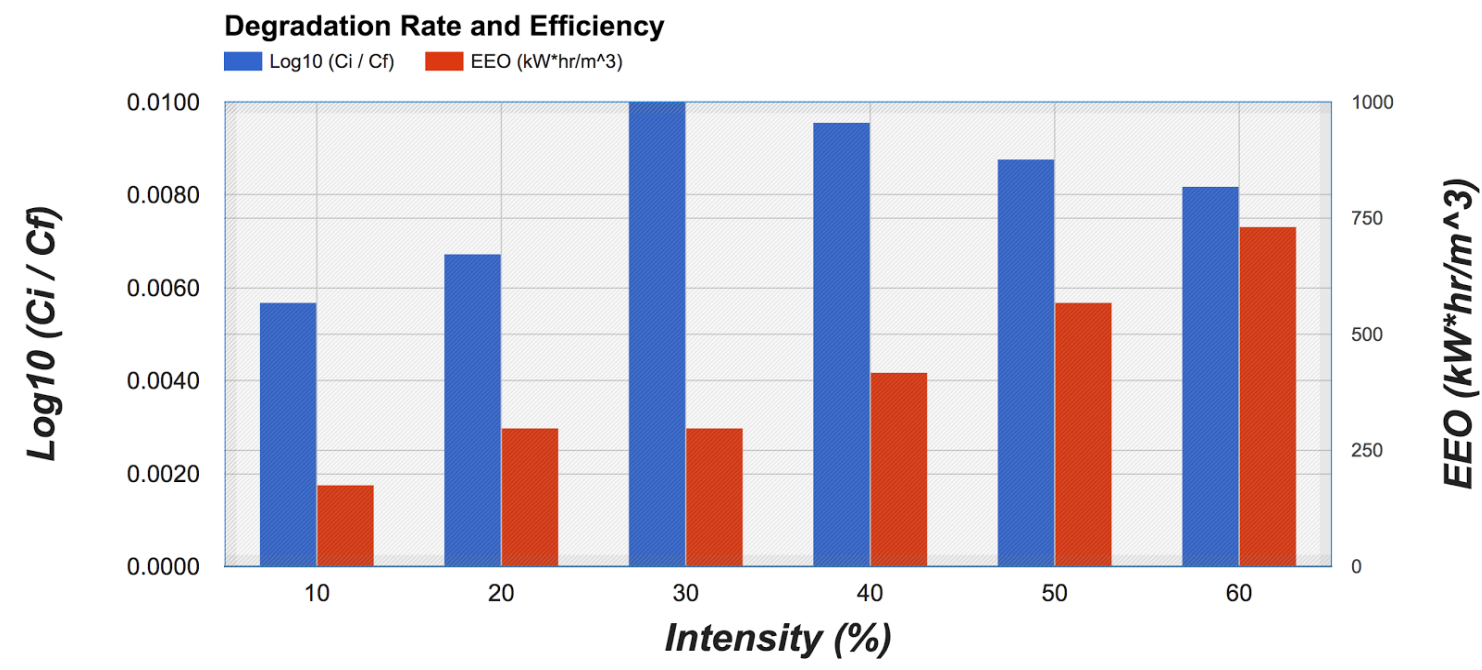

Figure 51: Evaluation of the system degradation rate (blue) and electrical energy per order (red) as a function of LED output intensity (as a percentage of full power).

It was found that the EEO monotonically increased with increasing intensity.

Interestingly, the pollutant degradation rate does not monotonically increase - there is a maximum degradation at $30 \%$ intensity. This is understood theoretically as a thermal effect, caused by the waste heat that is generated through the recombination of electrons and holes within the photocatalyst material. As the intensity of light increases, so does the temperature within the material, leading to an increased electron-hole recombination rate, and thus a lower EEO. This manifests in decreased reaction rates above a certain intensity threshold [19]. This point is important to determine, as it indicates that operation of the illumination system above $30 \%$ is not advisable since both efficiency and degradation rate decrease above this value. However, below $30 \%$ intensity, one has to choose if it is better to operate at high intensity (30\%) and achieve faster degradation but lower efficiency, or to operate at low intensity $(\leq 10 \%)$ and achieve higher efficiency but slower 
degradation. The answer is situationally dependent on the importance of efficiency for a particular application.

\subsubsection{Relationship of EEO to Fundamental Efficiency Parameters}

Because EEO is a measure of energy usage, it is inversely proportional to the system efficiency, as can be seen in Equation (17). This equation can be simplified when the power to the illumination system and initial concentration of pollutant remain fixed. In this case, a constant $(\epsilon)$ can be defined (Equation (41)) to group the constants relating to the illumination system settings and pollutant reaction rates. Doing so highlights the aspects of the EEO equation that are dependent on the photocatalyst material itself, i.e. the optical yield $(\chi)$ and quantum yield $(\phi)$. The result is Equation (42).

$$
\begin{aligned}
& \epsilon=\frac{0.640 P \sum k_{s_{i}}\left[S_{i}\right]}{G k_{C}} \\
& E E O=\frac{\epsilon}{\chi \phi}
\end{aligned}
$$

Notice that EEO and the efficiency parameters are inversely related. In fact, the product of $\chi$ and $\phi$ is directly relatable to photonic efficiency, $\xi$, discussed thoroughly in Chapter 1. However, it is elucidating to consider how these individual components contribute to energy usage by the system. The optical yield (sometimes referred to as the apparent quantum yield) is defined as the ratio of the rate of photon absorption by the photocatalyst material to the rate of photons incident on reaction chamber surface. The value of $\chi$ is, therefore, a number between 0 and 1 , and represents the ability of the photoreactor to collect photons generated by the illumination system. The quantum yield is defined as the ratio of the rate of generation of $\mathrm{OH} \bullet$ to the rate of photons absorbed by the photocatalyst material. Again, this a number between 0 and 1 , and represents the 
effectiveness of the photocatalyst for converting light into oxidizing species. This value is highly dependent on several physical properties of the photocatalyst material, such as its optical and electrical characteristics, surface morphology, crystallinity, defect density, and thickness.

Each of the modifications tested in this investigation has an effect on both $\chi$ and $\phi$. The decreased EEO can be attributed to three factors: 1) increased photocatalyst surface area, 2) decreased charge carrier recombination due to hole-trapping by $\mathrm{SiO}_{2}$ particles, 3) increased light absorption due to the decreased material band-gap, and 4) decreased electron-hole recombination rate within the photocatalyst due to charge separation at the anatase-rutile interface, and 5) a combination of increased light scattering and decreased reflection from the photocatalyst surface.

Additionally, the EEO results can be related directly to the activity optimization results in Section 5.1.2 in order to verify the predictions of these preliminary results. In particular, it was found that the modifications, applied to the $\mathrm{TiO}_{2}$-base film on the fused silica microscope slides, led to an increase of MB degradation rate of 523\%. When the same modifications were applied to the 3D photocatalyst cartridges, the system EEO was reduced by $81.9 \%$. This reduction has been directly associated with an increased efficiency in the combined process of harvesting of photons and conversion to oxidizing species $(\chi \bullet \phi)$. Since the EEO is inversely proportional the combined efficiency parameters it can be calculated that the material efficiency was increased by a factor of ( 1 $-0.819)^{-1}$ or $552 \%$. 
The fact that this improvement is even greater than that seen on the microscope slides is likely due to the geometrical differences between the two cases, since the increases scattering and decreased reflectance of the films have greater benefit for the $3 \mathrm{D}$ geometry of the cartridge that for the $2 \mathrm{D}$ geometry of the microscope slides. This is especially true because the angle of photon incidence for microscope slide experiments was approximately $0^{\circ}$, resulting in a lower dependence of degradation rate on scattering and reflection coefficients. 


\section{Conclusion}

The research that was presented investigates the connections between the energy use by photocatalytic water purification systems and the material properties of the photocatalyst used to instigate the reaction. This was motivated by the fact that implementation of photocatalytic water purification systems has been hampered by low efficiency of the conversion from UV photons to oxidizing species. To remedy this difficulty, a theoretical model was created as a basis for the design of a 3D photocatalyst material, which shows how the optical, geometrical, and electrical properties of photocatalyst material affect the photonic efficiency of the system, and is presented along with empirical validation of these concepts. Based on these results, a 3D material and complementary photoreactor system were designed, fabricated, and characterized.

In addition to the development of the photoreactor system itself, an analytical method was also developed in order to quantify the efficiency of the design using a figure-ofmerit technique established for comparison of AOP technologies. Once this system was in place, optimization of the system was carried out through modification of the photocatalyst surface and optimization of UV illumination intensity. This data was then used to validate the design through comparison with results with from other photoreactor systems.

The lowest EEO value achieved by the system was $54 \mathrm{kWh} / \mathrm{m}^{3}$. Although this is not the lowest EEO reported for all photocatalytic reactors, it is the lowest we have found to date for thin-film based photocatalyst materials. This is attributed to both the 3D cartridge design, rotational symmetry alignment of 3D material with the illumination system, as 
well as optimization of the photocatalyst film through thickness variation and nanoparticle implantation.

The EEO results were also related directly to the photoactivity of $\mathrm{TiO}_{2}$ films fabricated on fused silica microscope slides. It was found that modifications of film thickness and composition of the $\mathrm{TiO}_{2}$-base film led to an increase in photoactivity of $523 \%$. When the same modifications were applied to the 3D photocatalyst cartridges, the system EEO was reduced by $81.9 \%$. This reduction was associated with a $552 \%$ increase in efficiency for the combined process of harvesting of photons and conversion of charge carriers to oxidizing species at the catalytic surface.

In comparison to reported EEO values by other research groups, the EEO of the system designed in this investigation approaches values reported for nanoparticle slurry type systems. As slurry reactors are considered to represent an ideal efficiency for photocatalysis reactions, the fact that the obtained EEO values approach the values for slurry reactors, while also maintaining a durable and scalable photocatalyst material, suggests that this design can be considered a significant contribution to the field of water purification by photocatalysis.

Continuation of this research will include optimization of the 3D material through numerical simulation using finite element analysis. This work will include a variation of the geometrical arrangement of rod bundle to improve reaction kinetics and implementation of flow manipulation structures to enhance mixing within the reaction chamber. The theory presented in Chapter 1 will also be expanded, as described in Section 3.6, to generate quantitative predictions of the optimal photocatalyst film 
thickness. These results will the be tested experimentally through evaluation of the effects that these changes have on the system EEO. Once optimized, scaling of the system will be considered. The design of the photoreactor itself is intended to modular, such that multiple reactors can be connected in series or parallel, or any combination of the two: parallel connections will increase throughput, while series connections will create increased pollutant removal. 


\section{References}

[1] M. Perego, G. Seguini, G. Scarel, M. Fanciulli, and F. Wallrapp, "Energy band alignment at TiO2Si interface with various interlayers," J. Appl. Phys., vol. 103, no. 4, p. 43509, Feb. 2008.

[2] A. Fujishima and K. Honda, "Electrochemical Photolysis of Water at a Semiconductor Electrode," Nature, vol. 238, no. 5358, pp. 37-38, Jul. 1972.

[3] A. Fujishima, X. Zhang, and D. A. Tryk, "TiO2 photocatalysis and related surface phenomena," Surf. Sci. Rep., vol. 63, no. 12, pp. 515-582, Dec. 2008.

[4] E. Baur and A. Perret, "Über die Einwirkung von Licht auf gelöste Silbersalze in Gegenwart von Zinkoxyd," Helv. Chim. Acta, vol. 7, no. 1, pp. 910-915, Jan. 1924.

[5] C. Renz, "Über die Einwirkung von Oxyden auf Silbernitrat und Goldchlorid im Licht," Helv. Chim. Acta, vol. 15, no. 1, pp. 1077-1084, Jan. 1932.

[6] C. Goodeve and J. Kitchener, "Photosensitisation by titanium dioxide," Trans. Faraday Soc., vol. 34, pp. 570-579, 1938.

[7] M. C. Markham and K. J. Laidler, "A Kinetic Study of Photo-oxidations on the Surface of Zinc Oxide in Aqueous Suspensions," J. Phys. Chem., vol. 57, no. 3, pp. 363-369, Mar. 1953.

[8] S. Kato and F. Mashio, "TiO2 photocatalyzed oxidation of tetraline in liquid phase," Kogyo Kagaku Zasshi Jpn Ind Chem, vol. 67, pp. 1136-1140, 1964.

[9] I. S. McLintock and M. Ritchie, "Reactions on titanium dioxide; photo-adsorption and oxidation of ethylene and propylene," Trans. Faraday Soc., vol. 61, no. 0, pp. 1007-1016, Jan. 1965.

[10] S. N. Frank and A. J. Bard, "Heterogeneous photocatalytic oxidation of cyanide and sulfite in aqueous solutions at semiconductor powders," J. Phys. Chem., vol. 81, no. 15, pp. 1484-1488, Jul. 1977.

[11] M. A. Lazar, S. Varghese, and S. S. Nair, "Photocatalytic Water Treatment by Titanium Dioxide: Recent Updates," Catalysts, vol. 2, no. 4, pp. 572-601, Dec. 2012.

[12] A. R. Khataee, M. N. Pons, and O. Zahraa, "Photocatalytic degradation of three azo dyes using immobilized $\mathrm{TiO} 2$ nanoparticles on glass plates activated by UV light irradiation: Influence of dye molecular structure," J. Hazard. Mater., vol. 168, no. 1, pp. 451-457, Aug. 2009. 
[13] X. Zhao, "Analysis of Fungal Degradation of Azo Dyes," University of Georgia, 2004.

[14] R. Andreozzi, V. Caprio, A. Insola, and R. Marotta, "Advanced oxidation processes (AOP) for water purification and recovery," Catal. Today, vol. 53, no. 1, pp. 51-59, Oct. 1999.

[15] K. Hashimoto, H. Irie, and A. Fujishima, "TiO2 Photocatalysis: A Historical Overview and Future Prospects,” Jpn. J. Appl. Phys., vol. 44, no. 12R, p. 8269, Dec. 2005.

[16] H. Tong, S. Ouyang, Y. Bi, N. Umezawa, M. Oshikiri, and J. Ye, "Nanophotocatalytic Materials: Possibilities and Challenges," Adv. Mater., vol. 24, no. 2 , pp. 229-251, Jan. 2012.

[17] V. Diesen, "Heterogeneous TiO2 Photocatalysis: Fundamental Chemical Aspects and Effects of Solid Phase Alterations," 2013.

[18] M. Anpo and P. Kamat, Environmentally Benign Photocatalysts - Applications of Titanium Oxide-based Materials. .

[19] J.-M. Herrmann, "Fundamentals and misconceptions in photocatalysis," $J$. Photochem. Photobiol. Chem., vol. 216, no. 2-3, pp. 85-93, Dec. 2010.

[20] A. Fujishima, T. N. Rao, and D. A. Tryk, "Titanium dioxide photocatalysis," J. Photochem. Photobiol. C Photochem. Rev., vol. 1, no. 1, pp. 1-21, Jun. 2000.

[21] M. M. Huber, S. Canonica, G.-Y. Park, and U. von Gunten, "Oxidation of Pharmaceuticals during Ozonation and Advanced Oxidation Processes," Environ. Sci. Technol., vol. 37, no. 5, pp. 1016-1024, Mar. 2003.

[22] M. Pera-Titus, V. Garcia-Molina, M. A. Baños, J. Giménez, and S. Esplugas, "Degradation of chlorophenols by means of advanced oxidation processes: a general review," Appl. Catal. B Environ., vol. 47, no. 4, pp. 219-256, Feb. 2004.

[23] S. Esplugas, J. Giménez, S. Contreras, E. Pascual, and M. Rodriguez, "Comparison of different advanced oxidation processes for phenol degradation," Water Res., vol. 36, no. 4, pp. 1034-1042, Feb. 2002.

[24] J. J. Pignatello, E. Oliveros, and A. MacKay, "Advanced Oxidation Processes for Organic Contaminant Destruction Based on the Fenton Reaction and Related Chemistry," Crit. Rev. Environ. Sci. Technol., vol. 36, no. 1, pp. 1-84, Jan. 2006. 
[25] K. D. Kerri et al., Operation of wastewater treatment plants: a field study training program. Volume I Volume I. Sacramento: California State University], 2004.

[26] M. D. Swayne, G. H. Boone, D. Bauer, and J. S. Lee, "Wastewater in receiving waters at water supply abstraction points," 1980.

[27] J. Rice, A. Wutich, and P. Westerhoff, "Assessment of De Facto Wastewater Reuse across the U.S.: Trends between 1980 and 2008," Environ. Sci. Technol., vol. 47, no. 19, pp. 11099-11105, Oct. 2013.

[28] “National Tap Water Quality Database," Environmental Working Group, Dec. 2009.

[29] “Drinking Water Contaminants: List of Contaminants \& their MCLs.," U.S. Environmental Protection Agency (USEPA), 2009b.

[30] M. Pirnie, K. Linden, and J. Malley, "Ultraviolet Disinfection Guidence Manual for the Final Long Term 2 Enhanced Surface Water Treatment Rule." EPA, Office of Water, Nov-2006.

[31] E. L. Cates, Photocatalytic Water Treatment: So Where Are We Going with This? ACS Publications, 2017.

[32] K. Natarajan, T. S. Natarajan, H. C. Bajaj, and R. J. Tayade, "Photocatalytic reactor based on UV-LED/TiO2 coated quartz tube for degradation of dyes," Chem. Eng. $J$, vol. 178, pp. 40-49, Dec. 2011.

[33] D. Li, Q. Zhu, C. Han, Y. Yang, W. Jiang, and Z. Zhang, "Photocatalytic degradation of recalcitrant organic pollutants in water using a novel cylindrical multi-column photoreactor packed with TiO2-coated silica gel beads," J. Hazard. Mater., vol. 285, pp. 398-408, Mar. 2015.

[34] C. Lizama, J. Freer, J. Baeza, and H. D. Mansilla, "Optimized photodegradation of Reactive Blue 19 on TiO 2 and $\mathrm{ZnO}$ suspensions," Catal. Today, vol. 76, no. 2, pp. 235-246, 2002.

[35] G. E. Imoberdorf, G. Vella, A. Sclafani, L. Rizzuti, O. M. Alfano, and A. E. Cassano, "Radiation model of a TiO2-coated, quartz wool, packed-bed photocatalytic reactor," AIChE J., vol. 56, no. 4, pp. 1030-1044, Apr. 2010.

[36] N. Serpone, "Relative photonic efficiencies and quantum yields in heterogeneous photocatalysis," J. Photochem. Photobiol. Chem., vol. 104, no. 1-3, pp. 1-12, Apr. 1997. 
[37] J. R. Bolton, K. G. Bircher, W. Tumas, and C. A. Tolman, "Figures-of merit for the technical development and application of advanced oxidation processes."

[38] J. Marugán, D. Hufschmidt, G. Sagawe, V. Selzer, and D. Bahnemann, "Optical density and photonic efficiency of silica-supported TiO2 photocatalysts," Water Res., vol. 40, no. 4, pp. 833-839, Feb. 2006.

[39] L. C. Ferreira, M. S. Lucas, J. R. Fernandes, and P. B. Tavares, "Photocatalytic oxidation of Reactive Black 5 with UV-A LEDs," J. Environ. Chem. Eng., vol. 4, no. 1, pp. 109-114, Mar. 2016.

[40] H. D. Mansilla et al., "Photocatalytic EDTA degradation on suspended and immobilized TiO2," J. Photochem. Photobiol. Chem., vol. 181, no. 2-3, pp. 188194, Jul. 2006.

[41] M. A. Behnajady, N. Modirshahla, N. Daneshvar, and M. Rabbani, "Photocatalytic degradation of an azo dye in a tubular continuous-flow photoreactor with immobilized TiO2 on glass plates," Chem. Eng. J., vol. 127, no. 1-3, pp. 167-176, Mar. 2007.

[42] M. J. Benotti, B. D. Stanford, E. C. Wert, and S. A. Snyder, "Evaluation of a photocatalytic reactor membrane pilot system for the removal of pharmaceuticals and endocrine disrupting compounds from water," Water Res., vol. 43, no. 6, pp. 1513-1522, Apr. 2009.

[43] T. Luttrell, S. Halpegamage, J. Tao, A. Kramer, E. Sutter, and M. Batzill, "Why is anatase a better photocatalyst than rutile? - Model studies on epitaxial TiO2 films," Sci. Rep., vol. 4, Feb. 2014.

[44] G. Wang, L. Xu, J. Zhang, T. Yin, and D. Han, "Enhanced photocatalytic activity of $\mathrm{TiO} 2$ powders (p25) via calcination treatment," Int. J. Photoenergy, vol. 2012, p. e265760, Feb. 2012.

[45] Y. K. Kho, A. Iwase, W. Y. Teoh, L. Mädler, A. Kudo, and R. Amal, "Photocatalytic H2 Evolution over TiO2 Nanoparticles. The Synergistic Effect of Anatase and Rutile," J. Phys. Chem. C, vol. 114, no. 6, pp. 2821-2829, Feb. 2010.

[46] T. Kudo, Y. Nakamura, and A. Ruike, "Development of rectangular column structured titanium oxide photocatalysts anchored on silica sheets by a wet process," Res. Chem. Intermed., vol. 29, no. 6, pp. 631-639, Oct. 2003. 
[47] G. Vella, G. E. Imoberdorf, A. Sclafani, A. E. Cassano, O. M. Alfano, and L. Rizzuti, "Modeling of a TiO2-coated quartz wool packed bed photocatalytic reactor," Appl. Catal. B Environ., vol. 96, no. 3-4, pp. 399-407, Jun. 2010.

[48] H. Yamashita et al., "Preparation of Titanium Oxide Photocatalysts Anchored on Porous Silica Glass by a Metal Ion-Implantation Method and Their Photocatalytic Reactivities for the Degradation of 2-Propanol Diluted in Water," J. Phys. Chem. B, vol. 102, no. 52, pp. 10707-10711, Dec. 1998.

[49] X. Gao and I. E. Wachs, "Titania-silica as catalysts: molecular structural characteristics and physico-chemical properties," Catal. Today, vol. 51, no. 2, pp. 233-254, Jun. 1999.

[50] J. Krýsa, P. Novotná, Š. Kment, and A. Mills, "Effect of glass substrate and deposition technique on the properties of sol gel TiO2 thin films," J. Photochem. Photobiol. Chem., vol. 222, no. 1, pp. 81-86, Jul. 2011.

[51] C.-Y. Wu, Y.-L. Lee, Y.-S. Lo, C.-J. Lin, and C.-H. Wu, "Thickness-dependent photocatalytic performance of nanocrystalline $\mathrm{TiO} 2$ thin films prepared by sol-gel spin coating," Appl. Surf. Sci., vol. 280, pp. 737-744, Sep. 2013.

[52] E. Kozawa et al., "Photocatalytic activity of $\mathrm{TiO} 2$ particulate films prepared by depositing TiO2 particles with various sizes," J. Microencapsul., vol. 18, no. 1, pp. 29-40, Jan. 2001.

[53] J. Zhang et al., "Reconstruction of the (001) surface of $\mathrm{TiO} 2$ nanosheets induced by the fluorine-surfactant removal process under UV-irradiation for dye-sensitized solar cells," Phys. Chem. Chem. Phys. PCCP, vol. 14, no. 14, pp. 4763-4769, Apr. 2012.

[54] H. Tada and M. Tanaka, "Dependence of TiO2 photocatalytic activity upon its film thickness," Langmuir, vol. 13, no. 2, pp. 360-364, 1997.

[55] J. C. Yu, J. Yu, W. Ho, and J. Zhao, "Light-induced super-hydrophilicity and photocatalytic activity of mesoporous TiO2 thin films," J. Photochem. Photobiol. Chem., vol. 148, no. 1-3, pp. 331-339, May 2002.

[56] J. Lin et al., "3D Hierarchical Rutile TiO2 and Metal-free Organic Sensitizer Producing Dye-sensitized Solar Cells 8.6\% Conversion Efficiency," Sci. Rep., vol. 4, p. 5769, Aug. 2014. 
[57] M. Mazaheri, A. M. Zahedi, M. Haghighatzadeh, and S. K. Sadrnezhaad, "Sintering of titania nanoceramic: Densification and grain growth," Ceram. Int., vol. 35, no. 2 , pp. 685-691, Mar. 2009.

[58] R. Catabay, S. Fowler, and J. Jiao, "Annealing Effects on TiO2 Photocatalytic Degradation of Methylene Blue,” St Louis, Missouri, USA., 2017.

[59] H. Tada, "Photoinduced Oxidation of Methylsiloxane Monolayers Chemisorbed on TiO2," Langmuir, vol. 12, no. 4, pp. 966-971, Jan. 1996.

[60] K. Shinmou, N. Tohge, and T. Minami, "Fine-Patterning of ZrO2 Thin Films by the Photolysis of Chemically Modified Gel Films," Jpn. J. Appl. Phys., vol. 33, no. Part 2, No. 8B, pp. L1181-L1184, Aug. 1994.

[61] C. J. Brinker and G. W. Scherer, Sol-gel science: the physics and chemistry of solgel processing. Academic press, 2013.

[62] M. V. Liga, S. J. Maguire-Boyle, H. R. Jafry, A. R. Barron, and Q. Li, "Silica Decorated TiO2 for Virus Inactivation in Drinking Water - Simple Synthesis Method and Mechanisms of Enhanced Inactivation Kinetics," Environ. Sci. Technol., vol. 47, no. 12, pp. 6463-6470, Jun. 2013.

[63] V. H. Castrejón-Sánchez, E. Camps, and M. Camacho-López, "Quantification of phase content in TiO2 thin films by Raman spectroscopy," Superf. Vacio, vol. 27, no. 3, pp. 88-92, 2014.

[64] N. Ghobadi, "Band gap determination using absorption spectrum fitting procedure," Int. Nano Lett., vol. 3, no. 1, pp. 1-4, Jan. 2013.

[65] N. Serpone and A. Salinaro, "Terminology, relative photonic efficiencies and quantum yields in heterogeneous photocatalysis. Part I: Suggested protocol," Pure Appl. Chem., vol. 71, no. 2, pp. 303-320, 2009.

[66] N. Barbero and D. Vione, "Why Dyes Should Not Be Used to Test the Photocatalytic Activity of Semiconductor Oxides," Environ. Sci. Technol., vol. 50, no. 5, pp. 2130-2131, Mar. 2016.

[67] Y. Ohko, A. Fujishima, and K. Hashimoto, "Kinetic Analysis of the Photocatalytic Degradation of Gas-Phase 2-Propanol under Mass Transport-Limited Conditions with a TiO2 Film Photocatalyst," J. Phys. Chem. B, vol. 102, no. 10, pp. 17241729, Mar. 1998. 